\title{
Quantum entanglement via nilpotent polynomials
}

\author{
Aikaterini Mandilara* \\ Department of Physics, Washington University, Saint Louis, Missouri 63130, USA \\ Vladimir M. Akulin \\ Laboratoire Aime Cotton, CNRS, Campus d'Orsay, 91405, Orsay, France \\ Andrei V. Smilga ${ }^{\dagger}$ \\ SUBATECH, Université de Nantes, 4, rue Alfred Kastler, BP 20722-44307, Nantes-cedex 3, France
}

Lorenza Viola

Department of Physics and Astronomy, Dartmouth College, 6127 Wilder Laboratory, Hanover, New Hampshire 03755, USA

(Received 15 January 2006; published 30 August 2006)

\begin{abstract}
We propose a general method for introducing extensive characteristics of quantum entanglement. The method relies on polynomials of nilpotent raising operators that create entangled states acting on a reference vacuum state. By introducing the notion of tanglemeter, the logarithm of the state vector represented in a special canonical form and expressed via polynomials of nilpotent variables, we show how this description provides a simple criterion for entanglement as well as a universal method for constructing the invariants characterizing entanglement. We compare the existing measures and classes of entanglement with those emerging from our approach. We derive the equation of motion for the tanglemeter and, in representative examples of up to four-qubit systems, show how the known classes appear in a natural way within our framework. We extend our approach to qutrits and higher-dimensional systems, and make contact with the recently introduced idea of generalized entanglement. Possible future developments and applications of the method are discussed.

DOI: 10.1103/PhysRevA.74.022331

PACS number(s): 03.67.Mn, 03.65.Ud, 03.65.Fd
\end{abstract}

\section{INTRODUCTION: HOW TO CHARACTERIZE ENTANGLEMENT?}

Inseparability of quantum states of composite systems, discovered in the early days of quantum mechanics by Einstein, Podolsky, and Rosen [1] and named "entanglement" ("Verschränkung") by Schrödinger [2], became one of the central concepts of contemporary physics during the last decade. Entanglement plays now a vital role within quantum information science [3], representing both the defining resource for quantum communication-where it enables, in particular, nonclassical protocols such as quantum teleportation [4] and it leads to enhanced security in cryptographic tasks [5] — and a key ingredient for determining the efficiency of quantum computation algorithms [6,7]. In addition, studies of entanglement have also proved relevant to fields as different as atomic physics [8], quantum chaos [9-12], quantum phase transitions [13-20], and quantum networks [21].

According to the original definition, the description of entanglement relies on a specific partition of the composite physical system under consideration. However, such a system can often be decomposed into subsystems in many different ways, each of the subsystems possibly being a composite system by itself. In order to avoid ambiguity, given a partition of the composite system into $n$ subsystems, we call each of them an "element" and characterize it by a single, possibly collective, quantum number. Thus, the composite

\footnotetext{
*Present address: Laboratoire Aime Cotton, Orsay, France.

${ }^{\dagger}$ On leave of absence from ITEP, Moscow, Russia.
}

system is a collection of the elements. We call this collection an "assembly" in order to avoid confusion with an "ensemble," which is usually understood as a set of all possible realizations of a many-body system with an associated probability distribution over these realizations. The $i$ th element is assumed to have Hilbert space of dimension $d_{i}$. Qubit, qutrit, and qudit are widely used names for two-, three-, and $d$-level elements (with $d_{i}=2, d_{i}=3$, and $d_{i}=d$, respectively).

The term "entanglement" has a transparent qualitative meaning: A pure state of an assembly is entangled with respect to a chosen partition when its state vector cannot be represented as a direct product of state vectors of the elements. This notion can also be extended to generic mixed quantum states, whereby entanglement is defined by the inability to express the assembly density operator as a probabilistic combination of direct products of the density operators of the elements. Intuitively, one expects that in the presence of "maximum" entanglement [22], the states of all subsystems are completely correlated in such a way that a measurement performed on one part determines the states of all other parts.

The question is: How to quantitatively characterize entanglement for an assembly of many elements? One would like to have a measure ranging from zero for the product state to the maximum value for a maximally entangled state. This can be easily accomplished for the bipartite setting $n$ $=2$ that is, for an assembly consisting of two distinguishable elements $A$ and $B$, each of arbitrary dimension. In this case, the von Neumann $\operatorname{Tr}\left(\rho_{A} \ln \rho_{A}\right)$ or linear $\operatorname{Tr}\left(\rho_{A}^{2}\right)$ entropies based on the reduced density operator of either element, e.g., $\rho_{A}=\operatorname{Tr}_{B}(|\Psi\rangle\langle\Psi|)$, may be chosen as entanglement measures for a pure state $|\Psi\rangle$ of the assembly. However, already for the 
tripartite case $n=3$, characterizing entanglement becomes much harder. In fact, there are not one, but many different characteristics of entanglement and, apart from the question "How much?," one must also answer the question "In which way" $[23,24]$ are different elements entangled? In other words, apart from separability criteria [25], one also must introduce inequivalent entanglement measures [26-32] and entanglement classes [23,33-35].

The identification of appropriate measures is not unique, and is mostly dictated by convenience. The choice of classes may have a more solid ground based on group theory, since their definition is related to groups of local operations. These are operations applied individually to each of the elements and forming a subgroup of all possible transformations of the assembly state vector. One may resort to group-theoretical methods, which allow one to construct a set of invariants [33,36-38] under such local operations. Under the action of local transformations, which can be either unitary or, in the most general case, simply invertible, the state vector of the system undergoes changes, still remaining within a subset $\mathcal{O}$ (an orbit) of the overall Hilbert space $\mathcal{H}$. The dimension of the coset $\mathcal{H} / \mathcal{O}$, that is the number of independent invariants identifying the orbit, can be easily found for a generic quantum state. Still, there are singular classes of orbits that require special consideration. Their description depends critically on the number of elements and on the detailed structure of the assembly.

The numerical values of the complete set of invariants may be chosen as the "orbit markers" that provide one with an entanglement classification, although the choice of this set is not, in general, unique. It turns out that generally accepted measures of entanglement, such as concurrence [26] for two qubits $\left(n=2, d_{i}=2\right)$, and 3-tangle [23] for three qubits $(n=3$, $d_{i}=2$ ), are such invariants $[33,39]$. Generalizing these measures [33] to $n=4, d_{i}=2$ yields a connection between measures and invariants characterizing different classes. Their construction is not an easy task, and one needs to identify the invariants that are able to distinguish inequivalent types of entanglement [40]. Moreover, the invariants are usually highorder polynomials of the state amplitudes, with the maximum power growing linearly with the number of elements in the assembly. Therefore, they rapidly become rather awkward $[38,41]$. A partial (albeit not unambiguous) connection between the measures and classes is established by the requirement [42] for a measure to behave as a so-called monotone. This means it should be nonincreasing on average under the action of nonunitary invertible transformations of the elements, also known as Local Operations and Classical Communication, LOCC [43]. The complete classification problem remains unsolved even for relatively small assemblies (see, e.g., Ref. [44] for recent results on the five-qubit system).

Apart from polynomial-invariant constructions, other schemes have been proposed to describe multipartite entanglement, including those based on generalization of Schmidt decomposition [34,45-47], on invariant eigenvalues [33], on hyperdeterminants [35], and on expectation values of antilinear operators [40]. However, none of these suggestions have been fully tested for $n>4$ qubits or more than three qutrits $(n>3, d=3)$ [48]. Moreover, for the orbits of general invertible local transformations, the complete sets of invariants are unknown [49] for assemblies of $n$ qudits if $n$ $>3$ and/or $d>4$. Still, a number of physically reasonable suggestions $[28,32,50,51]$ for entanglement characterization have been attempted.

In this paper, we propose a different approach to entanglement characterization. We focus on the case where an assembly in a pure quantum state consists of distinguishable elements, leaving generalizations to mixed states and to indistinguishable elements for future studies. Our main aim is to construct extensive characteristics of entanglement. Thermodynamic potentials linearly scaling with the number of particles in the system offer examples of extensive characteristics widely employed in statistical physics. The free energy given by the logarithm of the partition function is a specific important example. We shall introduce similar characteristics for entangled states, in such a way that their values for a product state coincide with the sum of the values for unentangled groups of elements. Our technique is based on the notion of nilpotent variables and functions of these variables. An algebraic variable $x$ is called nilpotent if an integer $n$ exists, such that $x^{n}=0$. In our case, these variables are naturally associated with creation operators, where the logarithm function transforming products into sums plays a central role in the construction.

Our approach is based on three main ideas: (i) We express the state vector of the assembly in terms of a polynomial of creation operators for elements applied to a reference product state. (ii) Rather than working with the polynomial of nilpotent variables describing the state, we consider its logarithm, which is also a nilpotent polynomial. Due to the important role that this quantity will play throughout the development, we call this quantity the nilpotential henceforth, by analogy to thermodynamic potentials. (iii) The nilpotential is not invariant under local transformations, being different in general for different states in the same orbit. We therefore specify a canonic from of the nilpotential to which it can be reduced by means of local transformations. The nilpotential in canonic form is uniquely defined and contains complete information about the entanglement in the assembly. We therefore call this quantity the tanglemeter. The latter is, by construction, extremely convenient as an extensive orbit marker: the tanglemeter for a system consists of several not interacting, unentangled groups of elements equals the sum of tanglemeters of these groups.

Let us briefly explain these ideas, in the simplest example of $n$ qubits, which will be discussed in detail in Sec. II. An assembly of $n$ qubits is subject to the $\operatorname{su}(2)_{1} \oplus \cdots$ $\oplus \operatorname{su}(2)_{i} \cdots \oplus \operatorname{su}(2)_{n}$ Lie algebra of local transformations [52]. As a reference state, we choose the Fock vacuum that is, the state $|\mathrm{O}\rangle=|0,0, \ldots, 0\rangle$ with all the qubits being in the ground state. An arbitrary state of the assembly may be generated via the action of a polynomial $F\left(\sigma_{i}^{+}\right)$in the nilpotent operators $\sigma_{i}^{+}$on the Fock vacuum. Here, the subscript $i$ enumerates the qubits, and the operator $\sigma_{i}^{+}$creates the state $|1\rangle$ out of the state $|0\rangle$. Evidently, $\left(\sigma_{i}^{+}\right)^{2}=0$, since the same quantum state cannot be created twice. The family of all polynomials $F\left(\sigma_{i}^{+}\right)$forms a ring. We note that anticommuting nilpotent (Grassmann) variables are widely employed in quantum 
field theory [53] and in condensed matter physics [54]. However, the nilpotent variables introduced here commute with one another.

In order to uniquely characterize entanglement, it is necessary to select a convenient orbit marker. Following the idea of Ref. [45], we choose a state $\left|\Psi_{c}\right\rangle_{\mathcal{O}}$ lying in the orbit $\mathcal{O}$, which is the "closest" to the reference state $|\mathrm{O}\rangle$ in the inner product sense, that is $\left|\left\langle\mathrm{O} \mid \Psi_{c}\right\rangle_{\mathrm{O}}\right|=\max$. Once the state $\left|\Psi_{c}\right\rangle_{\mathrm{O}}$ is found, it is convenient to impose a nonstandard normalization condition, $\left|\left\langle\mathrm{O} \mid \Psi_{c}\right\rangle_{\mathrm{O}}\right|=1$. We call the resulting state $\left|\Psi_{c}\right\rangle_{\mathrm{O}}$ canonic. The latter is associated with the canonic form of the polynomial $F_{c}\left(\sigma_{i}^{+}\right)$, which begins with a constant term equal to 1 .

We mainly work not with $F_{c}$ by itself, but with the tanglemeter-a nilpotent polynomial $f_{c}=\ln F_{c}$ that can be explicitly evaluated by casting the logarithm function in a Taylor series of the nilpotent combination $F_{c}-1$. Since $\left(\sigma_{i}^{+}\right)^{2}$ $=0$, this series is a polynomial containing at most $2^{n}$ terms. Both the tanglemeter and nilpotential $(f=\ln F)$ resemble the eikonal, which is the logarithm of the regular semiclassical wave function in the position representation, multiplied by $-i$. The difference is that in our case no approximation is made: $f$ represents the logarithm of the exact state vector.

The nilpotential $f$ and the tanglemeter $f_{c}$ have several remarkable properties: (i) the tanglemeter provides a unique and extensive characterization of entanglement; (ii) a straightforward entanglement criterion can be stated in terms the cross derivatives $\partial^{2} f / \partial \sigma_{i}^{+} \partial \sigma_{j}^{+}$; (iii) the dynamic equation of motion for $f$ can be written explicitly and, suggestively, in the rather general case has the same form as the well-known classical Hamilton-Jacobi equation for the eikonal.

The paper is organized as follows. In Sec. II, we analyze in detail an assembly of $n$ qubits in terms of the nilpotent polynomials $F$ and $f$. We extend the notion of canonic forms to the group of reversible local transformations $\operatorname{SL}(2, \mathrm{C})$ and introduce the idea of entanglement classes. We conclude the section by presenting expressions relating the coefficients of $F_{C}$ and $f_{C}$ with known measures of entanglement. To avoid confusion, we note that the subscript $c$ corresponds to sucanonic forms in contrast to $C$, which corresponds to slcanonic forms. Details of the calculations and some proofs are given in Appendixes A and B, along with graphic representations of the entanglement topology.

In Sec. III, we consider the evolution of the nilpotent polynomials under the action of single-qubit and two-qubit Hamiltonians, and derive an equation of motion for the nilpotential, which is distinct from the Schrödinger equation. For one important particular case able to support universal quantum computation [55], we show that this equation has a form of the classical $n$-dimensional Hamilton-Jacobi equation. Describing quantum dynamics in terms of nilpotentials suggests a computational algorithm for evaluating tanglemeters, which can be performed by dynamically reducing the polynomials to the forms canonic under either $\mathrm{SU}(2)$ or $\mathrm{SL}(2, \mathrm{C})$ local transformations. In the example of a fourqubit assembly, we explicitly illustrate how to identify the resulting entanglement classes. This technique yields entanglement classes consistent with the results of Ref. [24]. The explicit analysis of these classes as well as details of derivation of the equation of motion are given in Appendixes $\mathrm{B}$ and $\mathrm{C}$.

In Sec. IV, we extend our technique to assemblies of $d$-level elements-starting from qutrits [56,57]. The CartanWeyl decomposition of the su $(d)$ algebras suggests a natural choice of nilpotent variables for qudits. For each element, we have $d-1=r$ variables representing commuting root vectors from the corresponding Lie algebra, which has rank $r$. For the illustrative case of two and three qutrits, we discuss possible choices of the canonic forms of the nilpotent polynomials. We further extend the approach to the case where the assembly partition may change as a result of the merging of elements, such that the new assembly consists of fewer number of elements with $d_{i} \neq d_{j}$, and consider transformations of nilpotent polynomials associated with such a change. Finally, we address a situation encountered in the framework of generalized entanglement [58,59], where the rank $r$ of the algebras of allowed local transformations is less than $d-1$. In other words, while the assembly is still assumed to be composed of a number of distinct elements, the group of local operations need not involve all possible local transformations. In such a situation, the proper nilpotent variables are more complicated than $\sigma^{+}$. In particular, they may have nonvanishing squares, etc., with only $d_{i}$ powers vanishing. In addition, unlike in the conventional setting, entanglement relative to the physical observables may exist not only among different subsystems, but also within a single element.

We conclude by summarizing our results and discussing possible developments and future applications of nilpotent polynomials and the tanglemeter.

\section{ENTANGLEMENT CHARACTERIZATION VIA NILPOTENT POLYNOMIALS}

Consider $n$ qubits in a generic pure state $|\Psi\rangle$,

$$
\begin{aligned}
|\Psi\rangle= & \sum_{\left\{k_{i}\right\}=0,1} \psi_{k_{n} k_{n-1} \cdots k_{1}}\left|k_{n}, k_{n-1}, \ldots, k_{1}\right\rangle=\psi_{00 \cdots 0}|0,0, \ldots, 0\rangle \\
& +\psi_{10 \cdots 0}|1,0, \ldots, 0\rangle+\psi_{01 \cdots 0}|0,1, \ldots, 0\rangle+\cdots \\
& +\psi_{11 \cdots 1}|1,1, \ldots, 1\rangle,
\end{aligned}
$$

specified by $2^{n}$ complex amplitudes $\psi_{k_{n} k_{n-1} \cdots k_{1}}$, i.e., by $2^{n+1}$ real numbers. The index $k_{i}=0,1$ corresponds to the ground or excited state of the $i$ th qubit, respectively. When we take normalization into account and disregard the global phase, there are $2^{n+1}-2$ real parameters characterizing the assembly state.

It is natural to expect that any measure characterizing the intrinsic entanglement in the assembly state remains invariant under unitary transformations changing the state of each qubit. A generic SU (2) transformation is the exponential of an element of the su(2) algebra,

$$
U=\exp \left[i\left(\sigma^{x} P^{x}+\sigma^{y} P^{y}+\sigma^{z} P^{z}\right)\right],
$$

where $\sigma^{x}, \sigma^{y}$, and $\sigma^{z}$ are Pauli matrices. It depends on the three real parameters $P^{x}, P^{y}$, and $P^{z}$. Such a transformation changes the amplitudes $\psi_{k_{n} k_{n-1} \cdots k_{1}}$ in Eq. (1), but preserves 
some combinations of these amplitudes - the invariants of local transformations. Thus, local transformations move the state along an orbit $\mathcal{O}$, while the values of the invariants serve as markers of this orbit.

The first relevant question is: What is the maximum number of real invariants required for the orbit identification, hence, for entanglement characterization? A generic SU(2) transformation represented by Eq. (2) depends on three real parameters. Therefore, for $n$ qubits the dimension of the coset $\mathcal{H} / \mathcal{O}$, that is the number of different real parameters invariant under local unitary transformations, reads $[45,50]$

$$
D_{s u}=2^{n+1}-3 n-2 .
$$

Mathematically, the counting in Eq. (3) corresponds to the number of invariants of the group $\otimes_{i} \mathrm{SU}_{i}(2) \otimes \mathrm{U}(1)$, where the factor $\mathrm{U}(1)$ describes multiplication by a common phase factor and the usual normalization condition $\langle\Psi \mid \Psi\rangle=1$ for the state vector is imposed. It is more convenient for us to reformulate the same problem as seeking for the invariants of $\otimes_{i} \mathrm{SU}_{i}(2) \otimes \mathrm{C}^{*}$, where $\mathrm{C}^{*}$ is the group of multiplication by an arbitrary nonzero complex number and no normalization condition is imposed. This will allow us to choose a representative on the orbit of $\mathrm{O}$ with a nonstandard normalization choice $\left\langle\Psi_{C} \mid \mathrm{O}\right\rangle=1$.

To be precise, the counting Eq. (3) is true for $n>2$ while the case $n=2$ is special: in spite of the fact that $2^{3}-3 \times 2$ $-2=0$, there is a nontrivial invariant of local transformations for two qubits. It has the form

$$
I=\psi_{00} \psi_{11}-\psi_{01} \psi_{10} .
$$

For a three-qubit system, five independent local invariants exist [39], namely three real numbers

$$
\begin{aligned}
& I_{1}=\psi_{k i j} \psi^{* p i j} \psi_{p m n} \psi^{* k m n}, \\
& I_{2}=\psi_{i k j} \psi^{* i p j} \psi_{m p n} \psi^{* m k n}, \\
& I_{3}=\psi_{i j k} \psi^{* i j p} \psi_{m n p} \psi^{* m n k},
\end{aligned}
$$

and the real and the imaginary part of a complex number,

$$
I_{4}+i I_{5}=\psi_{i j k} \psi^{i j p} \psi_{m n p} \psi^{m n k} .
$$

Here, with the summation over repeated indexes taking values 0 and 1 implicit, $\psi^{* i j k}$ denotes the complex conjugate of $\psi_{i j k}$, and $\epsilon^{i i^{\prime}}$ is the antisymmetric tensor of rank 2 . The quantity $2\left|I_{4}+i I_{5}\right|$ is also known by the name residual entanglement or 3-tangle $\tau$ Refs. [29,60].

Similar invariants can still be found for a four-qubit system. However, with increasing $n$, the explicit form of the invariants becomes less and less tractable and convenient for practical use. Moreover, no explicit physical meaning can be attributed to such invariants. We therefore suggest an alternative strategy, which is based on: (i) Specifying the canonic form of the state that unambiguously marks an orbit; (ii) characterizing this state with the help of coefficients of a nilpotent polynomial; (iii) considering the logarithm of this polynomial, the tanglemeter. Thus, we construct extensive invariants of local transformations as the coefficients of the tanglemeter that is, nilpotential of the canonic state.
In this section, we proceed with illustrating the main technical advantages of our description within the qubit setting, deriving an entanglement criterion, and explaining how the invariants constructed by our method are related to existing entanglement measures. We also analyze a case important for certain applications involving indirect measurements, where it is natural to consider a broader class of local transformations constrained only by the requirement of unit determinant. Specifically, we focus on the set of stochastic local operations assisted by classical communication [43], which is widely employed in quantum communication studies and protocols. For qubits, such transformations are known as SLOCC maps [31]. The latter do not necessarily preserve the normalization of state vectors. However, as suggested by Theorem 1 of Ref. [33], after a proper renormalization they are described by the complexification $\operatorname{sl}(2, \mathrm{C})$ of the $\mathrm{su}(2)$ algebra, such that the parameters $\left(P_{i}^{x}, P_{i}^{y}, P_{i}^{z}\right)$ specifying the transformation of Eq. (2) on each qubit are now complex numbers. The corresponding real positive invariants of local $\mathrm{SL}(2, \mathrm{C})$ transformations are monotones.

\section{A. Canonic form of entangled states}

In order to unambiguously attribute a marker to each orbit, we specify a canonic form of an entangled assembly state. To this end, we first identify a reference state $|\mathrm{O}\rangle$ as a direct product of certain one-qubit states. The latter may be chosen in an arbitrary way, but the choice $|0\rangle$, with the lowest energy level occupied, is the most convenient for our purposes. Thus, the reference state reads $|\mathrm{O}\rangle=|0, \ldots, 0\rangle$. Drawing parallels with quantum field theories and spin systems, we will call $|\mathrm{O}\rangle$ the "ground" or "vacuum" state. Then, following a suggestion of Ref. [45], by applying local unitary operations to a generic quantum state $|\Psi\rangle$, we can bring it into the "canonic form" $\left|\Psi_{c}\right\rangle$ corresponding to the maximum possible population of the reference state $|\mathrm{O}\rangle$. In other words, we apply a direct product $U_{1} \otimes \cdots \otimes U_{n}$ of transformations as in Eq. (2) to the state vector $|\Psi\rangle$, and choose real parameters $\left(P_{i}^{x}, P_{i}^{y}, P_{i}^{z}\right)$ to maximize $\left|\left\langle\mathrm{O}\left|U_{1} \otimes \cdots \otimes U_{n}\right| \Psi\right\rangle\right|^{2}$. The transformation $U_{1} \otimes \cdots \otimes U_{n}$ satisfying this requirement can be seen to be unique up to phase factors multiplying the upper states of each qubit. Modulo this uncertainty, the canonic state $\left|\Psi_{C}\right\rangle=U_{1} \otimes \cdots \otimes U_{n}|\Psi\rangle$ can serve as a valid orbit marker.

A generic unitary transformation of the $m$ th qubit, chosen in the form $\exp \left(i \sigma^{z} \phi_{m}\right) \exp \left(i \sigma^{x} g_{m}\right) \exp \left(i \sigma^{z} \varphi_{m}\right), \phi_{m}, g_{m}, \varphi_{m}$ $\in \mathbb{R}$, equivalent to Eq. (2), results in

$$
\begin{aligned}
\psi \cdots k_{m}=0 \cdots \rightarrow & \psi \cdots k_{m}=0 \cdots e^{-i \phi_{m}-i \varphi_{m}} \cos g_{m} \\
& +i \psi \cdots k_{m}=1 \cdots e^{i \varphi_{m}-i \phi_{m}} \sin g_{m}, \\
\psi & \\
& \\
& +i k_{m}=1 \cdots \rightarrow k_{m}=0 \cdots e^{i \phi_{m}-i \varphi_{m}} \sin g_{m} .
\end{aligned}
$$

Let us consider a generic infinitesimal local transformation of the state in the canonic form. By expanding Eq. (7) in series in $g_{m}$ up to the second order, one obtains 


$$
\begin{aligned}
\left\langle\mathrm{O}\left|\otimes_{i} U_{i}\right| \Psi\right\rangle \rightarrow & {\left[\left(1-\sum_{m} \frac{g_{m}^{2}}{2}\right) \psi_{0 \cdots 0}+i \sum_{m} g_{m} e^{2 i \varphi_{m}} \psi_{0 \cdots k_{m}=1 \cdots 0}\right.} \\
& \left.-\sum_{m>l} g_{m} g_{l} e^{2 i\left(\varphi_{m}+\varphi_{l}\right)} \psi_{0 \cdots k_{m}=1 \cdots k_{l}=1 \cdots 0}\right] \\
& \times \prod_{r} e^{-i\left(\phi_{r}+\varphi_{r}\right)}
\end{aligned}
$$

for the amplitude of the ground state. Since the parameters of the transformation are arbitrary, the condition of maximum ground-state population $|\langle O \mid \Psi\rangle|^{2}$ implies that the linear term in Eq. (8) vanishes,

$$
\psi_{0} \cdots k_{m}=1 \cdots 0=0, \quad \forall m .
$$

This gives $n$ complex conditions, and specifies $2 n$ out of $3 n$ real parameters of the local transformation that maps a generic state to the canonic form. The remaining $n$ parameters may be identified in a generic case with the phase factors $e^{-i\left(\phi_{r}+\varphi_{r}\right)}$, where one can set $\phi_{r}=0$ without loss of generality. Two remarks are in order.

(i) Special families of states of measure zero in the assembly Hilbert space may exist, for which the system of Eqs. (9) is degenerate and specifies less than $2 n$ parameters. The simplest example for $n=2$ is a Bell state, with $\psi_{00}=\psi_{11}=1 / \sqrt{2}$ and $\psi_{01}=\psi_{10}=0$. The combination of two transformations of the form (7) gives a state with the amplitudes

$$
\begin{aligned}
\psi_{00}^{\prime}= & \frac{e^{-i\left(\phi_{1}+\phi_{2}\right)}}{\sqrt{2}}\left(e^{-i\left(\varphi_{1}+\varphi_{2}\right)} \cos g_{1} \cos g_{2}\right. \\
& \left.-e^{i\left(\varphi_{1}+\varphi_{2}\right)} \sin g_{1} \sin g_{2}\right), \\
\psi_{10}^{\prime}= & i \frac{e^{i\left(\phi_{1}-\phi_{2}\right)}}{\sqrt{2}}\left(e^{-i\left(\varphi_{1}+\varphi_{2}\right)} \sin g_{1} \cos g_{2}\right. \\
& \left.+e^{i\left(\varphi_{1}+\varphi_{2}\right)} \cos g_{1} \sin g_{2}\right), \\
\psi_{01}^{\prime}= & i \frac{e^{i\left(\phi_{2}-\phi_{1}\right)}}{\sqrt{2}}\left(e^{-i\left(\varphi_{1}+\varphi_{2}\right)} \cos g_{1} \sin g_{2}\right. \\
& \left.+e^{i\left(\varphi_{1}+\varphi_{2}\right)} \sin g_{1} \cos g_{2}\right), \\
\psi_{11}^{\prime}= & \frac{e^{i\left(\phi_{1}+\phi_{2}\right)}}{\sqrt{2}}\left(e^{i\left(\varphi_{1}+\varphi_{2}\right)} \cos g_{1} \cos g_{2}\right. \\
& \left.-e^{-i\left(\varphi_{1}+\varphi_{2}\right)} \sin g_{1} \sin g_{2}\right) .
\end{aligned}
$$

One can see that the conditions $\psi_{01}^{\prime}=0$ and $\psi_{10}^{\prime}=0$ are not independent: They both give $g_{1}+g_{2}=0$ and $\varphi_{1}+\varphi_{2}=0$ with arbitrary $\phi_{1,2}$ (or, equivalently, $g_{1}-g_{2}=0, \varphi_{1}+\varphi_{2}=\pi / 2$ with arbitrary $\left.\phi_{1,2}\right)$.

The orbit of this special state has four parameters. On the other hand, for a generic canonic state with $\psi_{11} / \psi_{00}=\alpha,|\alpha|$ $\neq 1$, the conditions $\psi_{01}^{\prime}=\psi_{10}^{\prime}=0$ imply $g_{1}=g_{2}=0$ : only two phases $\phi_{1,2}+\varphi_{1,2}$ are arbitrary, whereas the transformed state is independent of the differences $\phi_{1,2}-\varphi_{1,2}$ in this case. When $n$ grows, the pattern of such special classes of states becomes more and more complicated. These families resemble "catastrophe manifolds" where infinitesimal variation of the state amplitudes $\psi$ result in a finite change of the local transformations reducing the state to the canonic form. Here, we shall not discuss this further, and restrict ourselves to the generic case.

(ii) As noticed in Ref. [39], the conditions (9) are necessary but not sufficient in general for the state to have maximum ground-state population $|\langle\mathrm{O} \mid \Psi\rangle|^{2}$. For example, an $n$ $=2$ state with $\left|\psi_{11}\right|>\left|\psi_{00}\right|$ and $\psi_{01}=\psi_{10}=0$ does not have the maximum ground-state population, although it satisfies Eq. (9): when $\left|\psi_{11}\right|$ starts to exceed $\left|\psi_{00}\right|$, finite "spin-flip" operations must be applied to both qubits to reduce the state to the canonic form.

For a generic $n$-qubit assembly state, the canonic form is unique up to $n$ phase factors $\phi_{m}+\varphi_{m}$, and the state may be characterized by $2^{n}-n-1$ complex ratios $\alpha_{k_{n} k_{n-1} \cdots k_{1}}$ $=\psi_{k_{n} k_{n-1} \cdots k_{1}} / \psi_{0 \cdots 0}$ with $\Sigma_{m} k_{m}>1$, whereas the amplitude of the vacuum state $\psi_{0} \cdots 0$, after being factored out, specifies the global phase and the normalization. We disregard these factors and normalize the state vector such that the amplitude of the reference state $\psi_{0} \cdots 0$ is set to 1 . Then the parameters $\alpha_{k_{n} k_{n-1} \cdots k_{1}}$ correspond to the amplitudes of the assembly states where at least two elements are excited. The number of real parameters characterizing the canonic form equals $2^{n+1}$ $-2 n-2$. It is worth mentioning that all $\left|\alpha_{k_{n} k_{n-1} \cdots k_{1}}\right|$ are invariant and, moreover, in the case $n \geqslant 3$, the ratios $\alpha_{k_{n} k_{n-1} \cdots k_{1}} \alpha_{l_{n} l_{n-1} \cdots l_{1}} / \alpha_{k_{n}^{\prime} k_{n-1}^{\prime} \cdots k_{1}^{\prime}} \alpha_{l_{n}^{\prime} l_{n-1}^{\prime \prime} \cdots l_{1}^{\prime}}$ are invariant if for each $m$ one of two conditions $k_{m}^{\prime}=k_{m}, l_{m}^{\prime}=l_{m}$ or $l_{m}^{\prime}=k_{m}, k_{m}^{\prime}$ $=l_{m}$ are satisfied. By specifying $n$ factors, we arrive at Eq. (3) for the maximum number of invariants characterizing entanglement.

Indeed, by an appropriate choice of the phase factors in Eq. (7), one can make a set of $n$ nonzero amplitudes $\alpha_{k_{n} k_{n-1} \cdots k_{1}}$ real and positive. For example, for a generic orbit one can make $n$ amplitudes $\alpha_{k_{n} k_{n-1} \cdots k_{1}}$ corresponding to the next-to-highest excited states real and positive, with $\Sigma_{m} k_{m}$ $=n-1$. In Fig. 1(a) we illustrate this for the simplest case $n$ $=3$ where the coefficients $\alpha_{011}, \alpha_{011}$, and $\alpha_{011}$ are chosen to be real and positive. As mentioned, the case $n=2$ is special, since the next-to-highest excited state amplitudes coincide with the first excited ones that vanish due to the requirement of Eq. (9). Thus, a single parameter $\alpha_{11}$ characterizing entanglement can always be chosen real and positive, in accordance with Eq. (10), where we have only one free phase factor $\varphi_{1}+\varphi_{2}$. Some further discussion is given in Appendix A.

Note that the determination of the canonic state for the orbit specified by an arbitrary state vector $|\Psi\rangle$ of an $n$-qubit assembly may be formulated as a standard quantum control problem: the task is to find the global maximum of the vacuum state population given by the functional $\mid\langle\mathrm{O}| U_{1}$ $\left.\otimes \cdots \otimes U_{n}|\Psi\rangle\right|^{2}$, starting from the initial state $|\Psi\rangle$. The space of the control parameters $\left\{P_{i}^{x}, P_{i}^{y}, P_{i}^{z}\right\}$ is $3 n$ dimensional. Without taking advantage of additional structure, the complexity of this procedure is in general exponential in $n$ [61]. A possibility to improve the efficiency of this search is based on exploiting the solution of a set of differential equations which is discussed in Sec. III D 3.

Note that the requirement of maximum vacuum-state population alone is insufficient for determination of the 
a)

$$
\begin{aligned}
& \Psi_{011^{\text {real }}}\left|011>\quad{\Psi 101^{\text {-real }}}_{\mid 101>}^{\Psi 110^{\text {-real }}}\right| 110>
\end{aligned}
$$

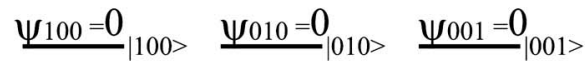

$$
\begin{aligned}
& \underline{\psi 000^{-r e a l}} \mid 000>
\end{aligned}
$$

b)

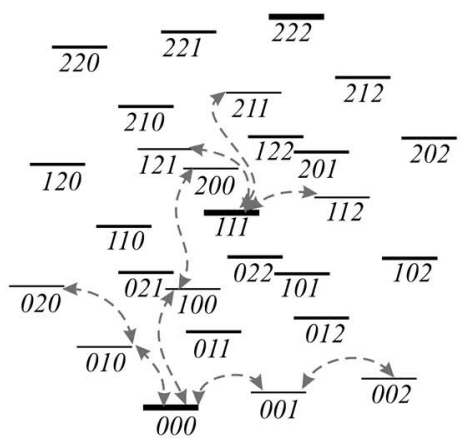

c)

$$
\underline{\beta_{1111}}=1_{111>}
$$

$\underline{\beta_{1110}=0}=1110>\underline{\beta_{1101}}=01101>\underline{\beta_{1011}=0}=1011>\quad \underline{\beta_{0111}=0}=0111>$

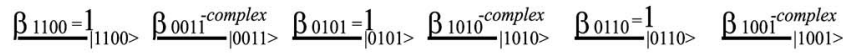

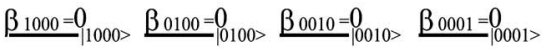

$$
\begin{aligned}
& \underline{\beta 0000=0}=000>
\end{aligned}
$$

FIG. 1. (a) Canonic form of the entangled state for three qubits. By a local transformation the amplitudes of the lowest excited states and the phases of the second highest exited states are set to zero. The amplitude of the lowest state is taken as the common factor determining the normalization and the global phase. (b) In the case of qudits, the group of local transformations is richer, and the canonic form can be chosen such that it corresponds to maximum population of the symmetric states $|k, k, k\rangle$ where $k=0, \ldots, d-1$. (c) The structure of the tanglemeter for a 4-qubit system in the case of $\mathrm{SL}(2, \mathrm{C})$ local transformations. The scaling factors $q_{i}$ can be chosen in many different ways, in particular such that some of the nonzero coefficients equal one.

canonic state of assemblies consisting of qudits with $d>2$. Indeed, a local unitary transformation not involving the vacuum state leaves this population intact, although it changes the amplitudes of other states. To eliminate such ambiguity, one needs to impose further constraints. As a possibility, one can maximize by a sequence of step the populations of $d-1$ states $|k, \ldots, k\rangle$, starting from $k=0$ and ending by $k=d-2$. In this sequential procedure, maximization of the amplitude of the state $|k, \ldots, k\rangle$ on the $(k+1)$ th step is done by a restricted class of local transformations belonging to the subgroup $\mathrm{SU}(d-k)$ that acts nontrivially only on the qudit states $|m\rangle$ with $m \geqslant k$. This algorithm leads to a generalization of the condition of Eq. (9): now, the amplitudes of all states coupled to the states $|k, \ldots, k\rangle$ by a single local transformation $\in \mathrm{SU}(d-k)$ such as $\psi_{0 \cdots 01}, \psi_{0 \cdots 02}, \ldots, \psi_{1 \cdots 12}, \ldots, \psi_{k \cdots k, m>k}$ but not $\psi_{k \cdots k, m<k}$ vanish. The action of these local transformations is indicated by dashed arrows in Fig. 1(b). The remaining nonzero amplitudes normalized to unit vacuum amplitude characterize entanglement in the assembly of qudits fairly unambiguously. One must still fix $n(d-1)$ phase factors of unitary transformations, but in analogy with the qubit case, this can be done by setting real and positive some $n(d-1)$ of $d^{n}-n d(d$ $-1) / 2-1$ nonvanishing amplitudes. In Sec. V we discuss this procedure in more detail.

\section{B. Nilpotent polynomials for entanglement characterization}

The amplitudes $\psi$ or $\alpha$ are not the most convenient quantities for characterizing entanglement, since they do not give an immediate idea about the entanglement structure. For instance, for two unentangled qubit pairs, each of which is in a Bell state, one finds

$$
\begin{aligned}
|\Psi\rangle= & \frac{1}{2}(|0,0\rangle+|1,1\rangle) \otimes(|0,0\rangle+|1,1\rangle)=\psi_{0000}|0,0,0,0\rangle \\
& +\psi_{1100}|1,1,0,0\rangle+\psi_{0011}|0,0,1,1\rangle+\psi_{1111}|1,1,1,1\rangle,
\end{aligned}
$$

where

$$
\begin{gathered}
\psi_{0000}=\psi_{1100}=\psi_{0011}=\psi_{1111}=1 / 2, \\
\alpha_{1100}=\alpha_{0011}=\alpha_{1111}=1 .
\end{gathered}
$$

In other words, though the system consists of two unentangled parts, each of which is characterized by only one parameter, three nonzero amplitudes $\alpha$ are present in the state vector. This is not convenient and a better description of the entanglement is desirable.

We now introduce a technique which serves this purpose. Consider a standard raising operator

$$
\sigma_{i}^{+}=\left(\begin{array}{ll}
0 & 1 \\
0 & 0
\end{array}\right),
$$

acting on the two-dimensional Hilbert space of the $i$ th qubit. Operators $\sigma_{i}^{+}$acting on different qubits commute. Since $\left(\sigma_{i}^{+}\right)^{2}=0$, these operators are nilpotent and they can be considered as nilpotent variables. Any quantum state $|\Psi\rangle$ as in Eq. (1) may be written in the form

$$
\begin{aligned}
|\Psi\rangle= & \left(\psi_{00 \cdots 0}+\psi_{00 \cdots 1} \sigma_{1}^{+}+\cdots+\psi_{01 \cdots 0} \sigma_{n-1}^{+}+\psi_{10 \cdots 0} \sigma_{n}^{+}\right. \\
& \left.+\psi_{00 \cdots 11} \sigma_{2}^{+} \sigma_{1}^{+} \cdots+\psi_{11 \cdots 0} \sigma_{n}^{+} \sigma_{n-1}^{+}+\cdots\right)|\mathrm{O}\rangle \\
= & \sum_{\left\{k_{i}\right\}=0,1} \psi_{k_{n} k_{n-1} \cdots k_{1}} \prod_{i=1}^{n}\left(\sigma_{i}^{+}\right)^{k_{i}}|\mathrm{O}\rangle
\end{aligned}
$$

where each nilpotent monomial $\Pi_{i=1}^{n}\left(\sigma_{i}^{+}\right)^{k_{i}}$ creates the basis state $\left|k_{n}, \ldots, k_{1}\right\rangle$ out of the vacuum state $|\mathrm{O}\rangle$. Let $F\left(\left\{\sigma_{i}^{+}\right\}\right)$be the nilpotent polynomial 


$$
\begin{aligned}
F\left(\left\{\sigma_{i}^{+}\right\}\right) & =\sum_{\left\{k_{i}\right\}=0,1} \alpha_{k_{n} k_{n-1} \cdots k_{1}} \prod_{i=1}^{n}\left(\sigma_{i}^{+}\right)^{k_{i}} \\
& =\sum_{\left\{k_{i}\right\}=0,1} \frac{\psi_{k_{n} k_{n-1} \cdots k_{1}}}{\psi_{00 \cdots 0}} \prod_{i=1}^{n}\left(\sigma_{i}^{+}\right)^{k_{i}}
\end{aligned}
$$

containing only the zeroth and first powers of each variable $\sigma_{i}^{+}$. A generic state $|\Psi\rangle$ normalized to unit vacuum amplitude $\psi_{0 \cdots 0}=1$ can thus be written as $F\left(\left\{\sigma_{i}^{+}\right\}\right)|\mathrm{O}\rangle$, with $F\left(\left\{\sigma_{i}^{+}\right\}\right)=1$ $+\alpha_{00 \cdots 1} \sigma_{1}^{+}+\cdots$.

Next, define the nilpotential $f\left(\left\{\sigma_{i}^{+}\right\}\right)$as given by the logarithm of $F\left(\left\{\sigma_{i}^{+}\right\}\right)$,

$$
f\left(\left\{\sigma_{i}^{+}\right\}\right)=\ln \left[F\left(\left\{\sigma_{i}^{+}\right\}\right)\right]=\sum_{\left\{k_{i}\right\}=0,1} \beta_{k_{n} k_{n-1} \cdots k_{1}} \prod_{i=1}^{n}\left(\sigma_{i}^{+}\right)^{k_{i}} .
$$

The coefficients $\beta_{k_{n} k_{n-1} \cdots k_{1}}$ and $\alpha_{k_{n} k_{n-1} \cdots k_{1}}$ can be explicitly related to each other by expanding $\ln F$ in a Taylor series around 1. This calculation requires at most $n$ operations consisting of multiplications of the polynomial $F-1$, which may generate an exponentially large $\left(\sim 2^{n}\right)$ number of terms. Note that $\beta_{00 \cdots 0}=0$ since $\alpha_{00 \cdots 0}=1$, so the nilpotential $f$ starts with the first-order terms. Both $F$ and $f$ contain a finite number of nilpotent terms, at most $2^{n}-1$, with the maximum-order term proportional to the monomial $\prod_{i=1}^{n} \sigma_{i}^{+}$given by the product of all the nilpotent variables. The canonic form of the state vector corresponds to a polynomial $F_{c}$,

$$
F_{c}=1+\alpha_{i j} \sigma_{i}^{+} \sigma_{j}^{+}+\cdots,
$$

which contains no linear monomials. The corresponding tanglemeter $f_{c}$, also contains no linear terms and reads

$$
f_{c}\left(\left\{\sigma_{i}^{+}\right\}\right)=\beta_{i j} \sigma_{i}^{+} \sigma_{j}^{+}+\cdots,
$$

with $\beta_{i j}=\alpha_{i j}$. The discussion in Sec. II A about the canonic form of the state vector applies to the tanglemeter as well. For most purposes, it suffices to employ the form which is unique up to phase changes of the nilpotent variables given by the local transformations $\sigma_{i}^{+} \mapsto \sigma_{i}^{+} e^{2 i \phi_{i}}$. The coefficients $\beta$ therefore remain invariant up to $n$ phase factors, unless these factors are specified by additional requirements. The phases $\phi_{i}$ may be chosen such that $n$ of nonzero coefficients $\beta$ are set real and positive. Should the tanglemeter of the generic state be defined unambiguously, we can require this for the coefficients $\beta_{k_{n} \cdots k_{1}}$ with $\Sigma_{i} k_{i}=n-1$, in the same way as it was done for $F$. In special cases, where one or several such coefficients equal zero, some other conditions on the phases may be imposed.

The tanglemeter $f_{c}\left(\left\{\sigma_{i}^{+}\right\}\right)$immediately allows one to check whether two groups $A$ and $B$ of qubits are entangled or not. The following criterion holds:

The entanglement criterion: The parts $A$ and $B$ of a binary partition of an assembly of $n$ qubits are unentangled iff

$$
\frac{\partial^{2} f_{c}\left(\left\{x_{i}\right\}\right)}{\partial x_{k} \partial x_{m}}=0, \quad \forall k \in A, \quad \forall m \in B .
$$

Thus, the subsystems $A$ and $B$ of the partition are disentangled iff $f_{A \cup B}=f_{A}\left(\left\{x_{\in A}\right\}\right)+f_{B}\left(\left\{x_{\in B}\right\}\right)$, and no cross terms are present in the tanglemeter. Note that the criterion of Eq.
(18) holds not only for the tanglemeter $f_{c}$, but for the nilpotential $f$ as well. However, we formulate the criterion in terms of $f_{c}$, because the coefficients $\beta$ of the tanglemeter are uniquely defined by construction.

\section{Examples: Canonic forms for two, three, and four qubits}

For two qubits, the result is immediate:

$$
f_{c}=\beta_{11} \sigma_{2}^{+} \sigma_{2}^{+}, \quad F_{c}=1+\alpha_{11} \sigma_{2}^{+} \sigma_{2}^{+}=1+\beta_{11} \sigma_{2}^{+} \sigma_{1}^{+},
$$

where the constant $\alpha_{11}=\beta_{11}$ may be chosen real. For three qubits, the canonic forms of $F$ and $f$ also differ only by the unity term,

$$
f_{c}=\beta_{3} \sigma_{2}^{+} \sigma_{1}^{+}+\beta_{5} \sigma_{3}^{+} \sigma_{1}^{+}+\beta_{6} \sigma_{3}^{+} \sigma_{2}^{+}+\beta_{7} \sigma_{3}^{+} \sigma_{2}^{+} \sigma_{1}^{+}=F_{c}-1 .
$$

Here, we have introduced a shorter notation by considering the indexes of $\beta$ as binary representation of decimal numbers, $011 \mapsto 3$, etc. One may use the fact that the variables $\sigma_{i}^{+}$ are defined up to phase factors to set $\beta_{3}, \beta_{5}$, and $\beta_{6}$ real. Expressing the invariants of Eqs. (5) and (6) via the parameters in the canonic form yields

$$
\begin{gathered}
I_{1}=1+2\left|\beta_{7}\right|^{2}\left(\beta_{3}^{2}+\beta_{5}^{2}+\beta_{6}^{2}\right)+\left|\beta_{7}\right|^{4}+2 \beta_{3}^{2}+\beta_{3}^{4}+2 \beta_{5}^{2} \beta_{6}^{2}, \\
I_{2}=1+2\left|\beta_{7}\right|^{2}\left(\beta_{3}^{2}+\beta_{5}^{2}+\beta_{6}^{2}\right)+\left|\beta_{7}\right|^{4}+2 \beta_{5}^{2}+\beta_{5}^{4}+2 \beta_{3}^{2} \beta_{6}^{2}, \\
I_{3}=1+2\left|\beta_{7}\right|^{2}\left(\beta_{3}^{2}+\beta_{5}^{2}+\beta_{6}^{2}\right)+\left|\beta_{7}\right|^{4}+2 \beta_{6}^{2}+\beta_{6}^{4}+2 \beta_{3}^{2} \beta_{5}^{2}, \\
I_{4}+i I_{5}=2\left(\beta_{7}^{2}+4 \beta_{3} \beta_{5} \beta_{6}\right) .
\end{gathered}
$$

This explicitly illustrates their linear independence. The tanglemeter for four qubits reads

$$
\begin{aligned}
f_{c}= & \beta_{3} \sigma_{2}^{+} \sigma_{1}^{+}+\beta_{5} \sigma_{3}^{+} \sigma_{1}^{+}+\beta_{9} \sigma_{4}^{+} \sigma_{1}^{+}+\beta_{6} \sigma_{3}^{+} \sigma_{2}^{+}+\beta_{10} \sigma_{4}^{+} \sigma_{2}^{+} \\
& +\beta_{12} \sigma_{4}^{+} \sigma_{3}^{+}+\beta_{7} \sigma_{3}^{+} \sigma_{2}^{+} \sigma_{1}^{+}+\beta_{13} \sigma_{4}^{+} \sigma_{3}^{+} \sigma_{1}^{+}+\beta_{11} \sigma_{4}^{+} \sigma_{2}^{+} a_{1}^{+} \\
& +\beta_{14} \sigma_{4}^{+} \sigma_{3}^{+} \sigma_{2}^{+}+\beta_{15} \sigma_{4}^{+} \sigma_{3}^{+} \sigma_{2}^{+} \sigma_{1}^{+},
\end{aligned}
$$

while the coefficients $\alpha_{i}$ of the polynomial $F_{c}$ differ from $\beta_{i}$ only at the last position

$$
\alpha_{15}=\beta_{15}+\beta_{3} \beta_{12}+\beta_{5} \beta_{10}+\beta_{9} \beta_{6} .
$$

One may note that the sums of the indexes of the factors in this expression are equal. The latter is a general feature for the relationship among the coefficients $\alpha$ and $\beta$ : the coefficients $\alpha$ are given by sums of terms, each of which contains a product of the coefficients $\beta$ where the sum of the indexes equals the index of $\alpha$. We also note that a proper set of invariants of $\otimes_{i=1}^{4} \mathrm{SU}_{i}(2) \otimes \mathrm{C}^{*}$ expressed via the components $\psi$ of the state vector can, in principle, be related to the tanglemeter coefficients, in analogy to the relation between Eqs. (5) and (6) and Eq. (21) for $\otimes_{i=1}^{4} \mathrm{SU}_{i}(2)$ invariants.

\section{Tanglemeter and entanglement classes for $\operatorname{SL}(2, \mathrm{C})$ operations}

We now take a larger class of local operations and consider arbitrary invertible linear transformations GL, instead 
of just the unitary transformations SU. Invertible transformations with nonzero determinant correspond in general to indirect measurements, i.e., measurements performed over an auxiliary system prepared in a certain quantum state after it has interacted with the system under consideration. Besides allowing the realization of measurements more general than projective von Neumann measurements [3], this procedure may serve as a tool for quantum control and quantum state engineering [62]. In the case where a single copy [63] of a quantum state is considered, the outcome of the measurement is not achieved with certainty. Therefore, a stochastic factor allowing for the outcome probability should be taken into account, where the resulting state vector must be renormalized accordingly. Since the normalization factor in the latter require an information about the initial state vector, such maps do not strictly speaking form a group. In our approach, we do not impose a normalization condition on the state vector whatsoever, and will be interested in finding the invariants [33] of the transformations belonging to the group $\mathcal{G}=\otimes_{i} \mathrm{SL}_{i}(2, \mathrm{C}) \otimes \mathrm{C}^{*}$, where $\mathrm{C}^{*}$ describes as before multiplication by an arbitrary nonzero complex number and the transformations $\in \mathrm{SL}_{i}(2, \mathrm{C})$ multiply the $i$ th qubit state by a $2 \times 2$ matrix of unit determinant. Another way to represent $\mathcal{G}$ is to express it as the product $\otimes_{i} \mathrm{GL}_{i}(2, \mathrm{C})$ and factorize it over $n-1$ redundant factors $\mathbb{C}^{*}$. The factors $\mathbb{C}^{*}$ in each $\mathrm{GL}_{i}(2, \mathrm{C})$ describe the same state transformation.

We emphasize that the change of the state vector renormalization does not result exclusively from the transformations belonging to $\mathrm{C}^{*}$, but from some $\operatorname{SL}(2, \mathrm{C})$ transformations as well-in particular, the transformations $\sim \exp q \sigma_{i}^{z}$, with complex $q$. Thus, considering just $\otimes_{i} \mathrm{SL}_{i}(2, \mathrm{C})$ instead of the full group $\mathcal{G}$ may not have an explicit physical sense. Still, we will do it sometimes to better reveal the mathematical structure of the results obtained. Since the local SL transformations comprise the key part of $\mathcal{G}$, following the established usage we will mainly refer to them and talk about sl-entanglement in assemblies subject to indirect local measurements.

Though local, SL operations can modify the set of quantities relevant for entanglement characterization. As SU $\subset$ SL, different su-orbits become equivalent under local SL transformations. In other words, the orbits of local SL transformations contain the su-orbits as subsets. Classification of sl-orbits reveals the entanglement which persists despite the indirect measurements. In order to distinguish this type of entanglement from the invariants under local unitary transformations, one may call it sl three-qubit assembly [23] shows that all generic quantum states belong to one orbit of $\mathcal{G}$, which includes the canonic Greenberger-Horne-Zeilinger (GHZ) state $(|000\rangle+|111\rangle) / \sqrt{2}[64]$. The invariant of Eq. (6), also known as 3-tangle $\tau$ [26], is different from zero only for this general orbit, and the value of $\tau$ calculated for the state amplitudes normalized to unit probability discriminates different su-orbits within this single general sl-orbit. States with $\tau=1$ can be reduced to GHZ state by local unitary transformations, while for other states, with $\tau<1$, indirect local measurements are required. Moreover, there are five singular orbits of $\mathcal{G}$ with $\tau=0$ that contain the states irreducible to the GHZ state. For four-qubit assemblies, the classification [24] becomes much more involved, but still it gives an idea about the types of entanglement and possible measures.

Each element of the $\mathrm{SL}_{i}(2, \mathrm{C})$ group, that is isomorphic to the Lorentz group SO $(3,1)$, involves six parameters. In the general case, the number of invariants,

$$
D_{\mathrm{sl}}=2^{n+1}-6 n-2,
$$

is less than that for unitary transformations Eq. (3). This counting is valid for $n \geqslant 4$, when the actions of different local operations are linearly independent. For $n=2,3$, where the number of the parameters in the group is larger than the number of the parameters in the state vector, and the result of some local SL are redundant, no invariants exist. In particular, for two qubits, any pure state is equivalent under $\mathcal{G}$ to a Bell state, and for three qubits to the GHZ state. For four qubits, there are six real invariants, for $n=5, D_{\mathrm{sl}}=32$, etc.

A smaller number of sl-invariants Eq. (24) as compared to that of su-invariants Eq. (3) implies that different su-orbits may belong to the same sl-orbit. In analogy to the su-canonic state, one must define a sl-canonic state as the marker of a sl-orbit. In contrast to the su case, where the canonic state has been defined by the condition of maximum reference state population, for SL transformations we introduce directly the canonic form of the tanglemeter. To this end, we impose the following conditions: in addition to the requirement of Eq. (9), i.e., all $n$ linear in $\sigma^{+}$terms of the nilpotential equal to zero, we require that all $n$ terms of $(n-1)$ th order vanish as well. Thus, the sl-tanglemeter takes the form

$$
f_{C}\left(\left\{\sigma_{i}^{+}\right\}\right)=\sum_{\Sigma_{i} k_{i} \neq\{1, n-1\}} \beta_{k_{n} k_{n-1} \cdots k_{1}} \prod_{i=1}^{n}\left(\sigma_{i}^{+}\right)^{k_{i}} .
$$

In this way, we have specified $4 n$ out of the $6 n$ real parameters of the local transformations that bring a given state to the sl-canonic form. We are left with $2 n$ parameters that must still be specified.

In contrast to the unitary case, where the nilpotent variables $\sigma^{+}$are defined up to arbitrary phase factors, for SL transformations the variables in Eq. (25) are defined up to a complex-valued scaling factor $\sigma_{i}^{+} \mapsto \sigma_{i}^{+} q_{i}$. One can then further specify the sl-tanglemeter by choosing these factors such that $n$ complex coefficients of the tanglemeter are set to unity. If convenient, however, one may impose another set of $n$ requirements.

As a first example, consider three qubits. The sltanglemeter for a generic three-qubit state reads

$$
f_{C}=\sigma_{3}^{+} \sigma_{2}^{+} \sigma_{1}^{+},
$$

where the coefficient is set to 1 by the scale freedom in the definition of the nilpotent variables. The corresponding state $F_{C}$ is nothing but the GHZ state. This shows again that all generic states belong to the same sl-orbit, which includes this state. There are, however, also three distinct singular classes of entangled states of measure zero [23] whose tanglemeters do not involve the product $\sigma_{1}^{+} \sigma_{2}^{+} \sigma_{3}^{+}$and have one of the following forms:

$$
f_{C}=\sigma_{2}^{+} \sigma_{1}^{+}+\sigma_{3}^{+} \sigma_{1}^{+},
$$




$$
\begin{aligned}
& f_{C}=\sigma_{3}^{+} \sigma_{1}^{+}+\sigma_{3}^{+} \sigma_{2}^{+}, \\
& f_{C}=\sigma_{2}^{+} \sigma_{1}^{+}+\sigma_{3}^{+} \sigma_{2}^{+} .
\end{aligned}
$$

In this classification, we have only taken into account the states whose tanglemeters involve all three $\sigma_{i}^{+}$such that no qubit is completely disentangled from the others.

For a generic four-qubit state one finds the sl-tanglemeter,

$$
\begin{aligned}
f_{C}= & \beta_{3} \sigma_{2}^{+} \sigma_{1}^{+}+\beta_{5} \sigma_{3}^{+} \sigma_{1}^{+}+\beta_{9} \sigma_{4}^{+} \sigma_{1}^{+}+\beta_{6} \sigma_{3}^{+} \sigma_{2}^{+}+\beta_{10} \sigma_{4}^{+} \sigma_{2}^{+} \\
& +\beta_{12} \sigma_{4}^{+} \sigma_{3}^{+}+\beta_{15} \sigma_{4}^{+} \sigma_{3}^{+} \sigma_{2}^{+} \sigma_{1}^{+},
\end{aligned}
$$

where the scaling factors $q_{i}$ of the variables $\sigma_{i}^{+}$can be specified such that this form becomes equivalent to the expression given in Theorem 2 of Ref. [24]:

$$
\begin{aligned}
f_{C}= & \beta_{3}\left(\sigma_{1}^{+} \sigma_{2}^{+}+\sigma_{3}^{+} \sigma_{4}^{+}\right)+\beta_{5}\left(\sigma_{1}^{+} \sigma_{3}^{+}+\sigma_{2}^{+} \sigma_{4}^{+}\right)+\beta_{6}\left(\sigma_{1}^{+} \sigma_{4}^{+}+\sigma_{2}^{+} \sigma_{3}^{+}\right) \\
& +\sigma_{1}^{+} \sigma_{2}^{+} \sigma_{3}^{+} \sigma_{4}^{+} .
\end{aligned}
$$

In Fig. 1(c), the structure of the four-qubit sl-tanglemeter is described with an alternative choice of the scaling factors.

It is worth mentioning that, though any generic nilpotential can be reduced to the canonic form of Eq. (25), this turns out to be impossible for some sets of states of measure zero, as it is already the case for three qubits. These sets, which play an important role for applications, can be grouped into special classes. Some of these classes are shown in Sec. III D 3 in the four-qubit example. There we also present an explicit algorithm for evaluating sl-tanglemeters based on the solution of dynamic equations with feedbacks imposed on the parameters of local transformations. This yields the special entanglement classes in a natural way, as singular stationary solutions.

We conclude this section by discussing the mathematical meaning of the canonic states. The renormalization of the state vector that follows the maximization of the reference state amplitudes by local SU transformations belongs to the group $\mathrm{C}^{*}$ of multiplication by a complex number $\kappa$. Therefore, strictly speaking, the applied transformations belong to the group $\otimes_{i} \mathrm{SU}_{i}(2) \otimes \mathrm{C}^{*}$. However, the group $\otimes_{i} \mathrm{SU}_{i}(2)$ does not affect the normalization of the state vector, while the requirement $\psi_{0}=1$ imposed on the canonic state uniquely specifies $\kappa$, thus allowing to introduce the tanglemeter as a characteristic of sl-orbits. In other words, once the condition $\psi_{\mathrm{O}}=1$ is satisfied, the group $\otimes_{i} \mathrm{SU}_{i}(2) \otimes \mathrm{C}^{*}$ becomes isomorphic to the group $\otimes_{i} \mathrm{SU}_{i}(2)$.

This is no longer the case for indirect measurements. Neither the group $\mathcal{G}$ nor its nontrivial part $\otimes_{i} \mathrm{SL}_{i}(2, \mathrm{C})$ conserves the state normalization. By imposing the requirement $\psi_{O}=1$, we mark an orbit of $\mathcal{G} / \mathrm{C}^{*}$, and thereby specify the structure of the canonic state given by the state amplitude ratios $\psi_{i} / \psi_{\mathrm{O}}$ expressed in terms of the sl-tanglemeter coefficients. However, a state of the same structure but with a different normalization may be physically achieved in many different ways - as a result of a single indirect measurement, or a sequence of two or more indirect measurements. The probability to obtain an outcome of the measurements that correspond to required $\mathcal{G} / \mathrm{C}^{*}$ transformation thus depends on the particular choice of the measurement procedure. Therefore, the complex factor $\kappa$ can be an arbitrary number, irrelevant to the values of the sl-tanglemeter coefficients.

However, when we consider just the nontrivial part $\otimes_{i} \mathrm{SL}_{i}(2, \mathrm{C})$ of $\mathcal{G}$, the factor $\kappa$ can bear certain physical significance. In fact, a transformation from $\otimes_{i} \mathrm{SL}_{i}(2, \mathrm{C})$ may bring a state initially normalized to unit probability to another one, which differs from the canonic state only by a factor $\kappa$. In this case the factor $\kappa$ is a uniquely defined function of the initial state [65]. When the transformation is unitary, $\kappa$ amounts to $1 / \sqrt{\sum_{i}\left|\psi_{i}\right|^{2}}$ where the amplitudes $\psi_{i}$ of the canonic state are normalized to unity reference state amplitude, as required. For nonunitary SL transformations, this quantity is different. Therefore, $\ln \left(\left|\kappa \sqrt{\sum_{i}\left|\psi_{i}\right|^{2}}\right|\right)$ can serve as a measure of nonunitarity of the transformation that discriminates different su-orbits belonging to the same $\mathcal{G}$-orbit.

\section{E. Relation to existing entanglement measures. Entanglement in four-qubit systems}

In general, there is no universal and precise definition of proper measures of entanglement [41], with the important exception of bipartite entanglement: as long as we are interested in entanglement between two parts $A$ and $B$ of a quantum system in a pure state, natural entanglement measures do exist. They are based on the reduced density operator $\rho_{A}$ of either part, obtained by tracing over the quantum numbers corresponding to the other part $B$. In particular, $S_{v N}$ $=-\operatorname{Tr}\left(\rho_{A} \ln \rho_{A}\right)$ and $S_{l}=1-\operatorname{Tr}\left(\rho_{A}^{2}\right)$, give the von Neumann and the linear entropies, respectively [3], as mentioned in the Introduction. Clearly, both characteristics can be directly related to the tanglemeter parameters. However, the explicit formulas giving these relations, which are simple for the case of two qubits

$$
\begin{gathered}
S_{l}=\frac{2\left|\beta_{11}\right|^{2}}{\left(1+\left|\beta_{11}\right|^{2}\right)^{2},} \\
S_{v N}=\ln \left(1+\left|\beta_{11}\right|^{2}\right)-\frac{\left|\beta_{11}\right|^{2}}{1+\left|\beta_{11}\right|^{2}} \ln \left(\left|\beta_{11}\right|^{2}\right),
\end{gathered}
$$

become awkward for larger numbers of qubits within the bipartition, as well as for higher-dimensional elements. This reflects the fact that the coefficients of nilpotent polynomials carry much more information about entanglement than the simple bipartite correlations captured by the entropy measures themselves.

Another useful entanglement measure, concurrence $C$, has been introduced in Ref. [26] in the context of mixed two-qubit states, and has been employed for constructing the residual entanglement $\tau$, as a measure characterizing threequbit pure-state entanglement and possibly beyond $[29,60]$. Both $C$ and $\tau$ may be expressed in terms of the amplitudes $\psi$ of the su-canonic state and in terms of the tanglemeter coefficients $\beta$, Eq. (20). The concurrence between the first and the second qubits reads 


$$
C_{12}=2|| \psi_{000} \psi_{110}|-| \psi_{101} \psi_{011}||=\frac{2|| \beta_{6}|-| \beta_{5} \beta_{3}||}{1+\beta_{6}^{2}+\beta_{5}^{2}+\beta_{3}^{2}+\left|\beta_{7}\right|^{2}} .
$$

The residual entanglement, or 3-tangle, has the form of a fourth-order polynomial in the amplitudes. For the canonic state, it reads

$$
\begin{aligned}
\tau_{3} & =4\left|\left(\psi_{000} \psi_{111}\right)^{2}+4 \psi_{000} \psi_{110} \psi_{101} \psi_{011}\right| \\
& =\frac{\left.4|| \beta_{7}\right|^{2}+4 \beta_{6} \beta_{5} \beta_{3} \mid}{\left(1+\beta_{6}^{2}+\beta_{5}^{2}+\beta_{3}^{2}+\left|\beta_{7}\right|^{2}\right)^{2}},
\end{aligned}
$$

which is proportional to the invariant $\left|I_{4}+i I_{5}\right|$ of Eqs. (6)-(21), divided by the normalization factor $\Sigma|\psi|^{2}=1+\beta_{6}^{2}$ $+\beta_{5}^{2}+\beta_{3}^{2}+\left|\beta_{7}\right|^{2}$. The presence of such a factor Eqs. (29) and (30) is due to the fact that these quantities are usually calculated for the state vector normalized to 1 , whereas the coefficients $\beta$ refer to the tanglemeter, which is the logarithm of the canonical state with the normalization $\psi_{000}=1$.

What are convenient measures for characterizing slentanglement? We have seen that all generic states of the assembly of three qubits belong to the same orbit of $\mathcal{G}$ and strictly speaking there are no invariant measures at all. However, the su-invariant $I_{4}+i I_{5}$ of Eq. (6) remains invariant under the restricted class of transformations $\otimes_{i=1}^{3} \mathrm{SL}_{i}(2, \mathrm{C})$, while the other SU-invariants $I_{1,2,3}$ of Eq. (5), which depend on both $\psi$ and $\psi^{*}$, change under SL transformations. Hence, in this restricted sense it may serve as a measure for slentanglement.

The measures characterizing the sl-entanglement for a four-qubit assembly can be constructed in a similar way. We take products of several factors $\sim \psi$ (but not the factors $\sim \psi^{*}$ ) and convolute it over $\mathrm{SU}(2)$-indexes with invariant tensors $\epsilon^{i i^{\prime}}$ [66]. The simplest combination,

$$
I^{(2)}=\psi_{i j k l} \psi^{i j k l},
$$

is sl-invariant and may be taken as a characteristic of slentanglement, remaining not invariant only with respect to the transformations $\in \mathbb{C}^{*}$. There are three different slinvariants $\sim \psi^{4}$,

$$
\begin{aligned}
& I_{13}^{(4)}=I_{24}^{(4)}=\psi_{i k j l} \psi^{j m j n} \psi_{o m p n} \psi^{\rho k p l}, \\
& I_{14}^{(4)}=I_{23}^{(4)}=\psi_{i k l j} \psi^{i m n j} \psi_{o m n p} \psi^{o k l p} .
\end{aligned}
$$

The ratios $I_{12}^{(4)} /\left(I^{(2)}\right)^{2}, I_{13}^{(4)} /\left(I^{(2)}\right)^{2}$, and $I_{14}^{(4)} /\left(I^{(2)}\right)^{2}$ are in addition invariant with respect to multiplication of the state vector by an arbitrary complex constant and thereby they are invariants of $\mathcal{G}$. Were these ratio linearly independent, they would give us a complete characterization of four-qubit entanglement, since the four-qubit sl-tanglemeter of Eq. (28) involves three complex parameters. However, they are not. The following identity,

$$
I_{12}^{(4)}+I_{13}^{(4)}+I_{14}^{(4)}=\frac{3}{2}\left(I^{(2)}\right)^{2}
$$

makes these quantities inconvenient for entanglement characterization.

We therefore turn to the sixth-order invariants and consider the following three independent combinations:

$$
\begin{aligned}
& I_{12}^{(6)}=\frac{1}{6}\left(\psi_{i n g d} \psi_{m r k o} \psi_{s j p h}-\psi_{i n g o} \psi_{m r k h} \psi_{s j p d}\right) \psi^{m r g d} \psi^{i n p h} \psi^{s j k o}, \\
& I_{23}^{(6)}=\frac{1}{6}\left(\psi_{i j p o} \psi_{m n g h} \psi_{s r k d}-\psi_{i j p d} \psi_{m n g o} \psi_{s r k h}\right) \psi^{m r g d} \psi^{i n p h} \psi^{s j k o}, \\
& I_{13}^{(6)}=\frac{1}{6}\left(\psi_{i j k h} \psi_{m n p d} \psi_{s r g o}-\psi_{i j g h} \psi_{m n k d} \psi_{s r p o}\right) \psi^{m r g d} \psi^{j n p h} \psi^{s j k o},
\end{aligned}
$$

whose differences give the invariants of Eq. (32) multiplied by $I^{(2)}$. The explicit form of these invariants for a generic state is awkward. However, they take a simple form for the canonic state, which allows us to explicitly relate them to the canonic amplitudes:

$$
\begin{aligned}
& \psi_{0000}=\frac{\sqrt{\sqrt{I_{13}^{(6)}+Q}+\sqrt{I_{23}^{(6)}+Q}+\sqrt{I_{12}^{(6)}+Q}-\left(I^{(2)}\right)^{3 / 2}}}{\sqrt{2}\left(I^{(2)}\right)^{1 / 4}}, \\
& \psi_{1100}=\psi_{0011}=\frac{\sqrt{\sqrt{I_{13}^{(6)}+Q}-\sqrt{I_{23}^{(6)}+Q}-\sqrt{I_{12}^{(6)}+Q}+\left(I^{(2)}\right)^{3 / 2}}}{2\left(I^{(2)}\right)^{1 / 4}}, \\
& \psi_{1001}=\psi_{1001}=\frac{\sqrt{\sqrt{I_{23}^{(6)}+Q}-\sqrt{I_{13}^{(6)}+Q}-\sqrt{I_{12}^{(6)}+Q}+\left(I^{(2)}\right)^{3 / 2}}}{2\left(I^{(2)}\right)^{1 / 4}}, \\
& \psi_{0101}=\psi_{1010}=\frac{\sqrt{\sqrt{I_{12}^{(6)}+Q}-\sqrt{I_{23}^{(6)}+Q}-\sqrt{I_{13}^{(6)}+Q}+\left(I^{(2)}\right)^{3 / 2}}}{2\left(I^{(2)}\right)^{1 / 4}}, \\
& \psi_{1111}=\frac{\sqrt{I_{13}^{(6)}+Q}+\sqrt{I_{23}^{(6)}+Q}+\sqrt{I_{12}^{(6)}+Q}+\left(I^{(2)}\right)^{3 / 2}}{2 \sqrt{2}\left(I^{(2)}\right)^{1 / 4} \sqrt{\sqrt{I_{13}^{(6)}}+Q}+\sqrt{I_{23}^{(6)}+Q}+\sqrt{I_{12}^{(6)}+Q}-\left(I^{(2)}\right)^{3 / 2}},
\end{aligned}
$$


where $Q$ is a root of a cubic equation

$$
\left(I_{13}^{(6)}+Q\right)\left(I_{23}^{(6)}+Q\right)\left(I_{12}^{(6)}+Q\right)=\left(I^{(2)}\right)^{3} Q^{2} .
$$

Equation (35) defines the canonic state vector form with respect to pure SL transformations. The canonic form for the whole group $\mathcal{G}$ is obtained from Eq. (35) by dividing by $\psi_{0000}$, with the ratios $\psi_{1100} / \psi_{0000}, \quad \psi_{1001} / \psi_{0000}$, and $\psi_{0101} / \psi_{0000}$ yielding the sl-tanglemeter coefficients $\beta_{3}, \beta_{5}$, and $\beta_{6}$, respectively. Different roots of the cubic equation (36) yield different sl-canonic states related by SL transformations. We can choose one particular root by minimizing the difference between the normalization of the canonic state and the initial normalization. Thus, the sl-entanglement in the four-qubit assembly can be completely characterized by three independent scale-invariant complex ratios,

$$
\begin{aligned}
& \beta_{3}=\frac{\sqrt{\sqrt{I_{13}^{(6)}+Q}-\sqrt{I_{23}^{(6)}+Q}-\sqrt{I_{12}^{(6)}+Q}+\left(I^{(2)}\right)^{3 / 2}}}{\sqrt{2} \sqrt{\sqrt{I_{13}^{(6)}+Q}+\sqrt{I_{23}^{(6)}+P}+\sqrt{I_{12}^{(6)}+Q}-\left(I^{(2)}\right)^{3 / 2}}}, \\
& \beta_{5}=\frac{\sqrt{\sqrt{I_{23}^{(6)}+Q}-\sqrt{I_{13}^{(6)}+Q}-\sqrt{I_{12}^{(6)}+Q}+\left(I^{(2)}\right)^{3 / 2}}}{\sqrt{2} \sqrt{\sqrt{I_{13}^{(6)}+Q}+\sqrt{I_{23}^{(6)}+Q}+\sqrt{I_{12}^{(6)}+Q}-\left(I^{(2)}\right)^{3 / 2}}}, \\
& \beta_{6}=\frac{\sqrt{\sqrt{I_{12}^{(6)}+Q}-\sqrt{I_{23}^{(6)}+Q}-\sqrt{I_{13}^{(6)}+Q}+\left(I^{(2)}\right)^{3 / 2}}}{\sqrt{2} \sqrt{\sqrt{I_{13}^{(6)}+Q}+\sqrt{I_{23}^{(6)}+Q}+\sqrt{I_{12}^{(6)}+Q}-\left(I^{(2)}\right)^{3 / 2}}},
\end{aligned}
$$

emerging from the invariants of Eqs. (31)-(34).

Let us ask now whether one can suggest a simple measure for characterizing sl-entanglement in four qubits, an analog of the 3-tangle for the three-qubit assembly. Two natural candidates may be considered. The first is the sum $\mathcal{S}_{1}=\Sigma|\psi|^{2}$ over the probabilities from Eq. (35), which gives the standard normalization of the canoniclike state. Once the invariants of Eqs. (31)-(34) are calculated for a state normalized to 1 , this sum shows the extent by which the SL transformation required for setting the state to the canonic form differs from a unitary transformation, whence $\left.|\ln \Sigma| \psi\right|^{2} \mid$ provides us with a measure of this nonunitary. This quantity discriminates different su-orbits that belong to the same sl-orbit in analogy to the 3-tangle, which differentiates between su-orbits within a single generic $\mathcal{G}$ orbit of 3-qubit assembly. Another natural measure, the sum of moduli squared of the sl-tanglemeter coefficients $\beta, \mathcal{S}_{2}=\Sigma|\beta|^{2}$, characterizes different $\mathcal{G}$ orbits. Now, $\mathcal{S}_{2}=0$ for the GHZ canonic state, whereas $\mathcal{S}_{2} \neq 0$ for all other states. Thus, this measure shows how close the orbit is to the GHZ orbit. The quantity $\Sigma\left|\beta-\beta^{\prime}\right|^{2}$ may serve as a measure characterizing the distance between two different $\mathcal{G}$-orbits.

The relations between the invariants $I^{(2)}, I^{(6)}$ and the measures $\mathcal{S}_{1}, \mathcal{S}_{2}$ are rather complicated. One may look for simpler measures that would not involve the exact calculation of the canonic state amplitudes Eqs. (35)-(37), which rely on the solution of a nonlinear equation (36). The simplest such measure is $I^{(2)}$. We have performed a numerical analysis by calculating $\left|I^{(2)}\right|$ for a variety of $\sim 10^{2}$ randomly chosen as- sembly states normalized to unity and comparing it with the nonunitarity measure $\mathcal{S}_{1}$. Interestingly, we observe a strong correlation between these quantities (see Fig. 2), whereas no marked correlation between $\mathcal{S}_{2}$ and any simple symmetric combination of $I^{(2)}$ and $I^{(6)}$ is found (data not shown).

\section{QUANTUM STATE OPERATIONS AND DYNAMICS IN TERMS OF THE NILPOTENT POLYNOMIALS}

In this section, we first describe the effects of local and gate transformations on the assembly state vector as algebraic manipulations of the corresponding polynomials $F$ and $f$. In principle, by applying a properly chosen sequence of finite local transformations, one may reduce a nilpotent polynomial to the canonic form, thereby specifying the tanglemeter. However, straightforwardly applying these transformations is not a very practical way to proceed, since it usually requires lengthy calculations.

We therefore turn to infinitesimal transformations, and derive the equations of motion describing the dynamics of the nilpotential under continuous local and gate operations. We show that, for an important class of Hamiltonians supporting universal quantum computation, the dynamic equation for the nilpotential acquires a well-known Hamilton-Jacobi form.

Next, we show how to determine the tanglemeter with the help of such an equation. To this end, a proper feedback is required, ensuring that the parameters of infinitesimal SU(2) or $\mathrm{SL}(2, \mathrm{C})$ transformations are adjusted to track current values of the nilpotential coefficients. The tanglemeter appears as a stable stationary solution, that is, a focus of the resulting equation. We illustrate this method for a four qubits system, by showing how to determine the sl-tanglemeter for a generic four-qubit state and how to identify a number of special classes that cannot be reduced to this form. For these classes, alternative natural tanglemeters are suggested.

\section{A. Local operations}

A local unitary transformation as in Eq. (2), acting on the $i$ th qubit may also be given in the equivalent form

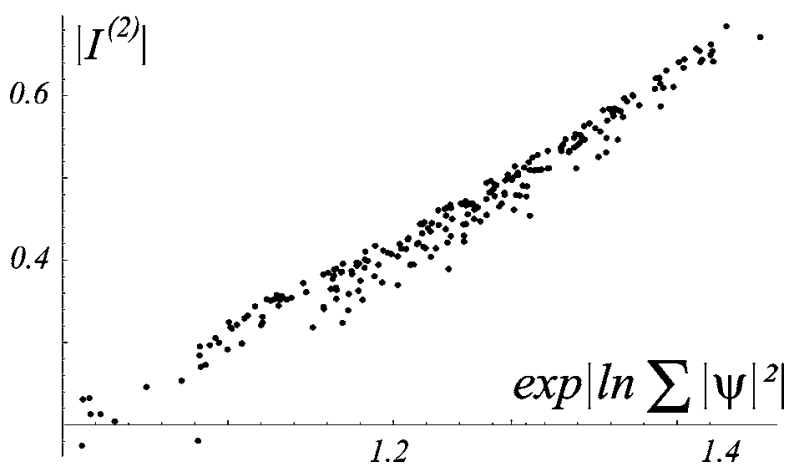

FIG. 2. The polynomial invariant $\left|I^{(2)}\right|$ plotted versus the nonunitarity measure $\left.\exp |\Sigma \ln | \psi\right|^{2} \mid$ for a set of $\sim 10^{2}$ randomly chosen pure states in a $n=4$ qubit assembly. 


$$
U_{i}=e^{A_{i} \sigma_{i}^{-}} e^{B_{i} \sigma_{i}^{z}} e^{C_{i} \sigma_{i}^{+}},
$$

which better suits the nilpotential polynomials formalism, since each step of the local transformation may be expressed as an operation linear in $\sigma$, that is,

$$
\begin{gathered}
e^{C_{i} \sigma_{i}^{+}}=1+C_{i} \sigma_{i}^{+}, \\
e^{B_{i} \sigma_{i}^{z}}=\cos \left(B_{i}\right)+\sin \left(B_{i}\right) \sigma_{i}^{z}, \\
e^{A_{i} \sigma_{i}^{-}}=1+A_{i} \sigma_{i}^{-} .
\end{gathered}
$$

The explicit expressions

$$
\begin{gathered}
A_{i}=\frac{\left(i P_{i}^{x}-P_{i}^{y}\right) \sin P}{P \cos P+i P_{i}^{z} \sin P}, \quad B_{i}=\ln \left(\cos P+\frac{i P_{i}^{z} \sin P}{P}\right), \\
C_{i}=\frac{\left(i P_{i}^{x}+P_{i}^{y}\right) \sin P}{P \cos P+i P_{i}^{z} \sin P}, \quad P=\sqrt{\sum_{\kappa=x, y, z}\left(P_{i}^{\kappa}\right)^{2}}
\end{gathered}
$$

relate the parameters in Eq. (2) and Eq. (38).

The transformations Eq. (38) act on the state vector $|\Psi\rangle$ $=F\left(\left\{\sigma_{i}^{+}\right\}\right)|\mathrm{O}\rangle$ and yield a transformed state $F^{\prime}\left(\left\{\sigma_{i}^{+}\right\}\right)|\mathrm{O}\rangle$. One can formalize the rules allowing one to obtain $F^{\prime}$ from $F$. Bearing in mind that $\sigma^{-}|0\rangle=0$ and $\sigma^{z}|0\rangle=-|0\rangle$, one can represent the action of $\sigma_{i}^{+}, \sigma_{i}^{z}, \sigma_{i}^{-}$as appropriate differential operations for the nilpotent variable $\sigma_{i}^{+}$. The application of the operator $\sigma_{i}^{+}$is straightforward-it is a direct multiplication: this operation eliminates the terms that were proportional to $\sigma_{i}^{+}$prior to the multiplication. The application of $\sigma_{i}^{-}$is a kind of inverse: it can be considered as a derivative with respect to the variable $\sigma_{i}^{+}$, which eliminates the terms independent of $\sigma_{i}^{+}$and makes the terms linear in $\sigma_{i}^{+}$independent of this variable [67]. Finally, the application of $\sigma_{i}^{z}$ changes the signs of the terms independent of $\sigma_{i}^{+}$, and leaves intact terms linear in $\sigma_{i}^{+}$. These actions are summarized by the following formulas:

$$
\begin{gathered}
\sigma_{i}^{+} F=\sigma_{i}^{+} F, \\
\sigma_{i}^{-} F=\frac{\partial F}{\partial \sigma_{i}^{+}}, \\
\sigma_{i}^{z} F=-F+2 \sigma_{i}^{+} \frac{\partial F}{\partial \sigma_{i}^{+}},
\end{gathered}
$$

while each unitary operation $U\left(\sigma_{i}^{x}, \sigma_{i}^{y}, \sigma_{i}^{z}\right)$ may be represented in terms of a differential operator $U\left(\sigma_{i}^{+}, \partial / \partial \sigma_{i}^{+}, 2 \sigma_{i}^{+} \partial / \partial \sigma_{i}^{+}-1\right)$.

By sequentially applying the three transformations of Eq. (38) to $F$, a local transformation may be interpreted as multiplication by an exponential function of $\sigma_{i}^{+}$, followed by a linear transformation $\sigma_{i}^{+} \mapsto e^{2 B_{i}}\left(A_{i}+\sigma_{i}^{+}\right)$of the variable $\sigma_{i}^{+}$ and multiplication by $e^{-B_{i}}$, leading to

$$
\begin{array}{rl}
U_{i} & F\left(\sigma_{1}^{+}, \ldots, \sigma_{i}^{+}, \ldots, \sigma_{n}^{+}\right) \\
& =e^{A_{i} \sigma_{i}^{-}} e^{B_{i} \sigma_{i}^{z}} F\left(\sigma_{1}^{+}, \ldots, \sigma_{i}^{+}, \ldots, \sigma_{n}^{+}\right) e^{C_{i} \sigma_{i}^{+}} \\
& =e^{A_{i} \sigma_{i}^{-}} e^{B_{i} \sigma_{i}^{z}} G\left(\sigma_{1}^{+}, \ldots, \sigma_{i}^{+}, \ldots, \sigma_{n}^{+}\right)
\end{array}
$$

$$
=e^{-B_{i}} G\left(\sigma_{1}^{+}, \ldots, e^{2 B_{i}} A_{i}+e^{2 B_{i}} \sigma_{i}^{+}, \ldots, \sigma_{n}^{+}\right) .
$$

As operations on different qubits commute, the Eq. (41) transformation straightforwardly generalizes to $n$ qubits.

Note that in order to cast $F$ into the canonic form,

$$
\begin{aligned}
F\left(\left\{\sigma_{i}^{+}\right\}\right) & =\sum_{\left\{k_{i}\right\}=0,1} \alpha_{k_{n} k_{n-1} \cdots k_{1}} \prod_{i=1}^{n}\left(\sigma_{i}^{+}\right)^{k_{i}} \mapsto F_{c}\left(\left\{\sigma_{i}^{+}\right\}\right) \\
& =\sum_{\left\{k_{i}\right\}=0,1 ; k_{1}+\cdots+k_{n} \neq 1} \alpha_{k_{n} k_{n-1} \cdots k_{1}} \prod_{i=1}^{n}\left(\sigma_{i}^{+}\right)^{k_{i}},
\end{aligned}
$$

one must solve a set of nonlinear equations for the parameters $A_{i}, B_{i}$, and $C_{i}$. This may be done explicitly only for at most four qubits, while for a larger system an efficient numerical technique is required. This task may be accomplished by an iterative procedure in the spirit of the Newton algorithm, that is, by consecutively applying a series $U_{n} \cdots U_{2} U_{1}$ of linear transformations $U_{i}$, each of which eliminates the terms linear in $\sigma_{i}^{+}$. However, this procedure may require infinitely many iterations, since a linear transformation applied to one of the $\sigma_{i}^{+}$may (and usually does) generate terms linear in other $\sigma_{j \neq i}^{+}$. In Sec. III D 3, we show how dynamic equations describing the evolution of the nilpotential $f$ under local transformations offer a better tool to solve this problem.

\section{B. Two-qubit gate operations}

Quantum gates are unitary transformations acting on finite subsets of qubits in the assembly. In particular, two-qubit gates $U_{i j}$ operate nontrivially on the pair $\{i, j\}$. Thanks to general universality results $[3,55]$, an arbitrary nonlocal transformation on $n$ qubits may be expressed as a finite sequence of arbitrary single-qubit operations and two-qubits operations drawn from a standard set, applied to both individual and pairs of qubits according to a certain quantum network. Thus, starting from an initial computational state, any state may be reached through the application of a quantum circuit built from gates in the set. We consider here the simplest choice for the standard two-qubit gate operation,

$$
\begin{aligned}
U_{i j} & =\exp \left[i t\left(\sigma_{i}^{+} \sigma_{j}^{-}+\sigma_{i}^{-} \sigma_{j}^{+}\right)\right]=\exp \left(i \frac{t\left(\sigma_{i}^{x} \sigma_{j}^{x}+\sigma_{i}^{y} \sigma_{j}^{y}\right)}{2}\right) \\
& =\cos ^{2} \frac{t}{2}+\sigma_{i}^{z} \sigma_{j}^{z} \sin ^{2} \frac{t}{2}+i \frac{\sigma_{i}^{x} \sigma_{j}^{x}+\sigma_{i}^{y} \sigma_{j}^{y}}{2} \sin t,
\end{aligned}
$$

depending on the single parameter $t \in \mathbb{R}$, where the tensor product symbol, $\sigma_{i}^{\kappa} \sigma_{j}^{\varkappa}=\sigma_{i}^{\kappa} \otimes \sigma_{j}^{\varkappa}$, is implicit.

Only the terms of $F$ that contain $\sigma_{i}^{+}$or $\sigma_{j}^{+}$are affected by the transformation Eq. (42). The terms that either do not contain these variables or are proportional to their product are left unchanged. Thus, the nilpotent polynomials $A_{i}$ $=A_{i}\left(\left\{\sigma_{k \neq i, j}^{+}\right\}\right)$undergo a unitary rotation,

$$
A_{i} \sigma_{i}^{+} \mapsto A_{i} \sigma_{i}^{+} \cos t+i A_{j} \sigma_{j}^{+} \sin t,
$$

in the same way as the components of a qubit state vector do under an SU(2) transformation. 


\section{Local and gate operations in terms of the nilpotential}

Equations (41)-(43) are special instances of general expressions for transforming the nilpotent polynomial $F$ under unitary operations - as we now consider.

As mentioned, using Eqs. (38)-(40), we may express the action of a unitary operation $U\left(\left\{\sigma_{i}^{+}, \sigma_{i}^{-}, \sigma_{i}^{z}\right\}\right)$ as a differential operator acting on $F$,

$$
F^{\prime}=U\left(\left\{\sigma_{i}^{+}, \frac{\partial}{\partial \sigma_{i}^{+}}, 2 \sigma_{i}^{+} \frac{\partial}{\partial \sigma_{i}^{+}}-1\right\}\right) F,
$$

whereas for the nilpotential one finds

$$
f^{\prime}=\log \left[U\left(\left\{\sigma_{i}^{+}, \frac{\partial}{\partial \sigma_{i}^{+}}, 2 \sigma_{i}^{+} \frac{\partial}{\partial \sigma_{i}^{+}}-1\right\}\right) e^{f}\right] .
$$

Note that a generic transformation Eq. (45) of an initially canonic polynomial does not necessarily result in another canonic polynomial.

Let us consider two particular cases of the general transformation of Eq. (45): (i) A local unitary operation as in Eq. (2), with $P_{i}^{x}=P \cos \phi, P_{i}^{y}=P \sin \phi, P^{z}=0$; (ii) the two-qubit gate in Eq. (42). They, respectively, transform the nilpotential according to

$$
\begin{gathered}
f^{\prime}=f+\ln \left(\cos P+i e^{i \phi} \frac{\partial f}{\partial \sigma_{i}^{+}} \sin P\right) \\
-i \sigma_{i}^{+} \frac{e^{i \phi}\left(\frac{\partial f}{\partial \sigma_{i}^{+}}\right)^{2}-e^{-i \phi}}{1+i e^{i \phi} \frac{\partial f}{\partial \sigma_{i}^{+}} \tan P} \tan P,
\end{gathered}
$$

and

$$
\begin{aligned}
f^{\prime}= & f+2 \sigma_{i}^{+} \sigma_{j}^{+}(1-\cos t) \frac{\partial^{2} f}{\partial \sigma_{i}^{+} \partial \sigma_{j}^{+}}-\left(\sigma_{j}^{+}-\sigma_{j}^{+} \cos t\right. \\
& \left.-i \sigma_{i}^{+} \sin t\right) \frac{\partial f}{\partial \sigma_{j}^{+}}-\left(\sigma_{i}^{+}-\sigma_{i}^{+} \cos t-i \sigma_{j}^{+} \sin t\right) \frac{\partial f}{\partial \sigma_{i}^{+}} \\
& -i \sigma_{i}^{+} \sigma_{j}^{+}\left[\frac{\sin 2 t}{2}\left(\frac{\partial f}{\partial \sigma_{i}^{+}}\right)^{2}+2 i \sin ^{2} t \frac{\partial f}{\partial \sigma_{i}^{+}} \frac{\partial f}{\partial \sigma_{j}^{+}}\right. \\
& \left.+\frac{\sin 2 t}{2}\left(\frac{\partial f}{\partial \sigma_{j}^{+}}\right)^{2}\right] .
\end{aligned}
$$

\section{Equations of motion for the nilpotential}

Consider now an infinitesimal unitary transformation $U$ $=1-i d t H$ not necessarily local. The increment $\Delta f$ of the nilpotential $f$ suggested by the Eq. (46) reads

$$
\Delta f=\ln \left(U e^{f}\right)-\ln \left(e^{f}\right)=\ln \left(1-i d t e^{-f} H e^{f}\right) .
$$

This yields the following dynamic equation for $f$ :

$$
i \frac{\partial f}{\partial t}=e^{-f} H e^{f}
$$

which we discuss in detail in the rest of this section.

\section{Local Hamiltonians}

We begin with the case of a local Hamiltonian,

$$
\begin{aligned}
H & =\sum_{i} P_{i}^{x}(t) \sigma_{i}^{x}+P_{i}^{y}(t) \sigma_{i}^{y}+P_{i}^{z}(t) \sigma_{i}^{z} \\
& =\sum_{i} P_{i}^{-}(t) \sigma_{i}^{+}+P_{i}^{+}(t) \sigma_{i}^{-}+P_{i}^{z}(t) \sigma_{i}^{z},
\end{aligned}
$$

where $P_{i}^{ \pm}=P_{i}^{x} \pm i P_{i}^{y}$, and we first separately consider only the term $H_{i}=P_{i}^{-}(t) \sigma_{i}^{+}+P_{i}^{+}(t) \sigma_{i}^{-}+P_{i}^{z}(t) \sigma_{i}^{z}$ in the sum. Upon substituting it in Eq. (49) and splitting the nilpotential $f$ on the right-hand side in two parts, the part $f_{0}=f-\sigma_{i}^{+} \partial f / \partial \sigma_{i}^{+}$independent of $\sigma_{i}^{+}$, and the part $f_{1}=\sigma_{i}^{+} \partial f / \partial \sigma_{i}^{+}$linear in $\sigma_{i}^{+}$, we obtain

$$
i \frac{\partial f}{\partial t}=e^{-\sigma_{i}^{+} \partial f / \partial \sigma_{i}^{+}} H_{i} e^{\sigma_{i}^{+} \partial f / \partial \sigma_{i}^{+}}
$$

The part $f_{0}$ commutes with the derivatives entering the Hamiltonian, and therefore cancels. Substitution of Eq. (40) into the Hamiltonian of Eq. (50), followed by expansion over the nilpotent variable $\sigma_{i}^{+}$, results in

$$
i \frac{\partial f}{\partial t}=-P_{i}^{z}+P_{i}^{-} \sigma_{i}^{+}+\left(2 P_{i}^{z} \sigma_{i}^{+}+P_{i}^{+}\right) \frac{\partial f}{\partial \sigma_{i}^{+}}-P_{i}^{+} \sigma_{i}^{+}\left(\frac{\partial f}{\partial \sigma_{i}^{+}}\right)^{2} .
$$

Straightforward generalization of this equation to the case of the Hamiltonian Eq. (50) yields

$$
i \frac{\partial f}{\partial t}=\sum_{i=1}^{n}\left[-P_{i}^{z}+P_{i}^{-} \sigma_{i}^{+}+\left(2 P_{i}^{z} \sigma_{i}^{+}+P_{i}^{+}\right) \frac{\partial f}{\partial \sigma_{i}^{+}}-P_{i}^{+} \sigma_{i}^{+}\left(\frac{\partial f}{\partial \sigma_{i}^{+}}\right)^{2}\right] .
$$

Another equivalent form of the same equation reads

$$
\begin{aligned}
i \frac{\partial f}{\partial t}= & \sum_{i=1}^{n}\left[P_{i}^{z}\left(2 f_{i} \sigma_{i}^{+}-1\right)+P_{i}^{x}\left(f_{i}+\sigma_{i}^{+}-f_{i}^{2} \sigma_{i}^{+}\right)\right. \\
& \left.+i P_{i}^{y}\left(f_{i}-\sigma_{i}^{+}-f_{i}^{2} \sigma_{i}^{+}\right)\right],
\end{aligned}
$$

where we denote $f_{i}=\partial f / \partial \sigma_{i}^{+}$. Note that the coefficients $P_{i}^{x, y, z}$ can explicitly depend on time. Also, note that the right-hand side of Eqs. (52)-(54) does not depend on the constant term in $f$. In other words, the latter, though evolving with time by itself, does not affect the evolution of the "essential" coefficients in the nilpotential in front of the nilpotent variables and their products.

\section{Binary interactions}

We now consider the two-qubit interaction

$$
H=\sum_{i, j, \kappa, \varkappa} G_{i j}^{\kappa \varkappa}(t) \sigma_{i}^{\kappa} \sigma_{j}^{\varkappa}, \quad \kappa, \varkappa=+,-, z .
$$

Note that the local transformations Eq. (54) may be absorbed into the time dependence of the coupling coefficients $G_{i j}^{\kappa \varkappa}(t)$ by simply passing to the interaction representation. In order to achieve universal evolution in this representation, one needs to consider all nine coefficients $G_{i j}^{\kappa \varkappa}$ characterizing the 
interaction of Eq. (55) between a pair $\{i, j\}$ of qubits as being different from zero. An alternative way is to choose a representation where the tensor $G_{i j}^{\kappa \varkappa}(t)$ in Eq. (55) takes the form of a diagonal spherical tensor with respect to the upper indexes. In this representation, the Hamiltonian

$$
\begin{aligned}
H= & \sum_{i, \kappa} P_{i}^{\kappa}(t) \sigma_{i}^{\kappa}+\sum_{i, j} G_{i j}^{+-}(t)\left(\sigma_{i}^{+} \sigma_{j}^{-}+\sigma_{i}^{-} \sigma_{j}^{+}\right)+\sum_{i, j} G_{i j}^{z z}(t) \sigma_{i}^{z} \sigma_{j}^{z} \\
& +\sum_{i, j} G_{i j}^{++}(t)\left(\sigma_{i}^{+} \sigma_{j}^{+}+\sigma_{i}^{-} \sigma_{j}^{-}\right)
\end{aligned}
$$

apart of the local operations of Eq. (53) involves also the binary interactions determined by the three real parameters $G_{i j}^{z z}(t), G_{i j}^{+-}(t)=G_{i j}^{-+}(t)$, and $G_{i j}^{++}(t)=G_{i j}^{--}(t)$.

The explicit forms of the equations of motion Eq. (49) for the Hamiltonians Eqs. (55) and (56) are rather awkward. We note, however, that universal evolution is achieved [55] with an even simpler Hamiltonian

$$
H=\sum_{i} P_{i}^{+}(t) \sigma_{i}^{+}+\sum_{i} P_{i}^{-}(t) \sigma_{i}^{-}+\sum_{i<j} G_{i j}(t)\left(\sigma_{i}^{+} \sigma_{j}^{-}+\sigma_{i}^{-} \sigma_{j}^{+}\right),
$$

with $P_{i}^{+}(t)=P_{i}^{-}(t)^{*}$, which depends on a smaller set of operators, $\sigma_{i}^{+}, \sigma_{i}^{-}$, and $\left(\sigma_{i}^{+} \sigma_{j}^{-}+\sigma_{i}^{-} \sigma_{j}^{+}\right)$. Repeated commutators of these operators satisfy the Lie-algebraic bracket generation condition for complete controllability, that is, all-order commutators span the full space of Hermitian operators for the assembly, and thus ensures universal evolution. It therefore suffices to specify the form of Eq. (49) for the Hamiltonian of Eq. (57).

In Appendix $\mathrm{C}$, we derive the corresponding equation of motion for $f$. It reads

$$
\begin{aligned}
i \frac{\partial f}{\partial t}= & \sum_{i}\left[P_{i}^{-}(t) \sigma_{i}^{+}+P_{i}^{+}(t) \frac{\partial f}{\partial \sigma_{i}^{+}}\left(1-\sigma_{i}^{+} \frac{\partial f}{\partial \sigma_{i}^{+}}\right)\right] \\
& +\sum_{i \neq j} G_{i j}(t) \sigma_{j}^{+} \frac{\partial f}{\partial \sigma_{i}^{+}}\left(1-\sigma_{i}^{+} \frac{\partial f}{\partial \sigma_{i}^{+}}\right) .
\end{aligned}
$$

Note that Eq. (58) formally resembles the Hamilton-Jacobi equation for the mechanical action of classical systems with the Hamiltonian

$H=\sum_{i}\left[P_{i}^{-}(t) x_{i}+P_{i}^{+}(t) p_{i}\left(1-x_{i} p_{i}\right)\right]+\sum_{i \neq j} G_{i j}(t)\left[x_{j} p_{i}\left(1-x_{i} p_{i}\right)\right]$,

where $p_{i}=\partial f / \partial \sigma_{i}^{+}$play the role of momenta, while $x_{i}=\sigma_{i}^{+}$are the coordinates. Comparing with the standard classical Hamilton-Jacobi equation, the only essential difference is the factor $\mathbf{i}$ multiplying the time derivative and the presence of complex parameters that may be interpreted as timedependent forces and masses. After cumbersome calculations which take into account the fact that the constants of motions entering the action function are nilpotent variables, one may reproduce the finite transformations of Eqs. (46) and (47).

\section{Dynamic equations for the nilpotential and construction of $S U(2)$ and $\mathrm{SL}(2, \mathrm{C})$ tanglemeters}

The dynamic equation, Eq. (58), suggests an algorithm for evaluating the tanglemeter. The basic idea is to properly adjust the parameters $P_{i}$ of the local transformations in Eq. (58) so to track the current values of the tanglemeter's coefficients. To this end, we fix the terms linear in $\sigma_{i}^{+}$in the local Hamiltonian of Eq. (50) as

$$
P_{i}^{-}=\left(P_{i}^{+}\right)^{*}=-i \beta_{i},
$$

where

$$
\beta_{i}=\left.\frac{\partial f}{\partial \sigma_{i}^{+}}\right|_{\sigma \rightarrow 0},
$$

are the coefficients of the linear terms in the nilpotential at a given time. From Eqs. (58)-(60), we find the evolution of these coefficients under local transformations,

$$
\frac{\partial \beta_{i}}{\partial t}=-\beta_{i}+\sum_{i=1}^{n}\left(\left.\beta_{j}^{*} \frac{\partial^{2} f}{\partial \sigma_{j}^{+} \partial \sigma_{i}^{+}}\right|_{\sigma \rightarrow 0}-\beta_{i}\left|\beta_{i}\right|^{2}\right) .
$$

When $f$ is close to $f_{c}$, the matrix of the second derivatives $M_{i j}=\left.\left(\partial^{2} f / \partial \sigma_{j}^{+} \partial \sigma_{i}^{+}\right)\right|_{\sigma \rightarrow 0}$ can be explicitly expressed in terms of the "second excited state amplitudes," $\psi_{0} \cdots k_{i}=1, k_{j}=1, \cdots, 0$, which enter Eq. (8), and were introduced when discussing the su-canonic form of states. According to Eq. (17),

$$
M_{i j}^{C}=\left.\frac{\partial^{2} f_{c}}{\partial \sigma_{j}^{+} \partial \sigma_{i}^{+}}\right|_{\sigma \rightarrow 0}=\beta_{i j}=\alpha_{i j}=\psi_{0 \cdots k_{i}=1, k_{j}=1, \cdots, 0} / \psi_{0, \cdots, 0} .
$$

The condition of the maximum reference state population for a state in the canonic form suggested by Eqs. (8) and (9) implies that the population increment,

$$
\begin{aligned}
\delta \rho_{|\mathrm{O}\rangle} & =\left|\psi_{0 \cdots 0}\right|^{2}\left(\left|1-\frac{1}{2} \sum_{i, j} g_{i}\left(e^{2 i\left(\varphi_{i}+\varphi_{j}\right)} M_{i j}+\delta_{i j}\right) g_{j}\right|^{2}-1\right) \\
& =-\operatorname{Re}\left|\psi_{0} \cdots\right|^{2} \sum_{i, j} g_{i}\left(e^{2 i\left(\varphi_{i}+\varphi_{j}\right)} M_{i j}+\delta_{i j}\right) g_{j},
\end{aligned}
$$

is always negative. As the phases $\varphi_{i}$ are arbitrary, the eigenvalues of $M_{i j}$ lie within the unit circle, hence their real parts lie in the interval $(-1,1)$. Therefore, Eq. (62) linearized in the vicinity of the canonic state,

$$
\begin{aligned}
& \frac{\partial \beta_{i}}{\partial t}=-\beta_{i}+\sum_{j} M_{i j} \beta_{j}^{*}, \\
& \frac{\partial \beta_{i}^{*}}{\partial t}=-\beta_{i}^{*}+\sum_{j} M_{i j}^{*} \beta_{j},
\end{aligned}
$$

has a stable stationary point at $\left\{\beta_{i}\right\}=0$. This implies that the coefficients $\beta_{i}$ standing in front of the linear terms $\beta_{i} \sigma_{i}^{+}$in the nilpotential tend to zero exponentially. The presence of the nonlinear terms $-\beta_{i}\left|\beta_{i}\right|^{2}$ further accelerates this trend, as negative quantities $-\left|\beta^{2}\right|$ are added to the negative eigenvalues of the matrix $M_{i, j}-\delta_{i, j}$ [68]. Therefore, an arbitrary nilpotential $f$ subject to the local transformation with the parameters of the Hamiltonian Eq. (50) chosen according to 
the feedback conditions of Eqs. (60) and (61), rapidly converges to the tanglemeter $f_{c}$. The problem of finding an efficient numerical algorithm for determining the tanglemeter for large assemblies is thereby solved. Verification that the outcome indeed corresponds to the global maximum of the reference state population should finalize the procedure. Note, however, that the maximum vacuum state population obtained with local transformations corresponds to the maximum population of the ground state for each qubit. On the other hand, for a given set of single-qubit density matrices, the local operations maximizing the ground state population of each qubits are uniquely defined. Therefore, the only maximum of the reference state population is the global one. This ensures that, no matter what the initial state is, the procedure indeed converges to the canonic state, and no verification is required.

A procedure of reducing the nilpotential to the canonic form may be carried out also for SL transformations. At the first stage of this procedure, we reduce it to the su-canonic form, so that the terms linear in $\sigma_{i}^{+}$vanish. Then we apply SL operations. An element of the $\mathrm{SL}(2, \mathrm{C})$ group can be represented as $\exp \left\{i t\left(P_{i}^{-} \sigma_{i}^{+}+P_{i}^{+} \sigma_{i}^{-}+P_{i}^{z} \sigma_{i}^{z}\right)\right\}$, where $P_{i}^{-}$and $P_{i}^{+}$are no longer complex conjugates and $P_{i}^{z}$ is also a complex number.

Finding the sl-canonic state may also be formulated as a control problem, based on feedback. We choose the parameters $P_{i}$ in the Hamiltonian of Eq. (53) in such a way that the terms in the nilpotential involving the monomials of order one and of order $n-1$ in $\sigma_{i}^{+}$would decrease exponentially with time. To this end, we may choose at this stage $P_{i}^{z}=0$, and impose two conditions: (i) the condition

$$
P_{j}^{-}=-\left.\sum_{i=1}^{n} P_{i}^{+} \frac{\partial^{2} f}{\partial \sigma_{i}^{+} \partial \sigma_{j}^{+}}\right|_{\sigma \rightarrow 0}=-\sum_{i=1}^{n} P_{i}^{+} \beta_{i, j},
$$

expressing $P_{i}^{-}$via $P_{i}^{+}$, which is preserving the nilpotential in the form of a tanglemeter; and (ii) the condition

$$
\begin{aligned}
\left.i \frac{\partial^{n-1} f}{\prod_{i \neq j} \partial \sigma_{i}^{+}}\right|_{\sigma \rightarrow 0}= & -P_{j}^{+} \frac{\partial^{n} f}{\prod_{i} \partial \sigma_{i}^{+}} \\
& +\left.\sum_{m=1}^{n} P_{k}^{+} \frac{\partial^{n-1}}{\prod_{i \neq m} \partial \sigma_{i}^{+}}\left[\sigma_{i}^{+}\left(\frac{\partial f}{\partial \sigma_{i}^{+}}\right)^{2}\right]\right|_{\sigma \rightarrow 0},
\end{aligned}
$$

which ensures the exponential decrease of all $n$ coefficients in front of the second-highest order terms.

Unfortunately, no immediate physical meaning seems to be attributable to the requirements of vanishing of the sltanglemeter coefficients in front of $(n-1)$ th order terms, in contrast to the case of SU transformations, where vanishing of the first-order terms indicates maximum ground state population. This requirement is suggested by symmetry reasons: $n$ complex conditions are imposed on $n$ complex coefficients of the same type. After having eliminated the monomials of orders 1 and $n-1$, we can specify the scaling parameters $P_{i}^{z}$ such that $n$ additional conditions are imposed on the tangemeter coefficients. For example, we can set to unity the coefficients in front of the highest order term and set $(n-1)$ coefficients in front of certain monomials equal to $(n-1)$ coefficients of other monomials. Within the group $\mathcal{G}$, multiplication by a complex number $\kappa \in \mathrm{C}^{*}$ allows one to normalize the canonic state to unit vacuum-state amplitude, thereby removing the constant term in the sl-tanglemeter $f_{C}$ Eq. (25).

The condition in Eq. (65) for $P_{j}^{+}$is written implicitly as a set of $n$ linear equations. These equations can be solved for generic states as we will show in the next section in the four-qubit case, Eq. (66). However, they have no solution when the determinant of the system vanishes. Such singularities correspond to singular classes of entangled states, and require special consideration.

\section{Example: Classes and sl-tanglemeters for 4 qubits}

In order to explicitly illustrate the procedure for evaluating the sl-tanglemeter via the dynamic equation, the starting point is provided by the su-tanglemeter, given in Eq. (22). As the latter has 11 complex coefficients $\beta_{i}$, one must solve a system of 11 first-order nonlinear differential equations. Instead of presenting this awkward system explicitly, we schematically depict in Fig. 3 contributions to the time derivatives of the $\beta_{i}$ which are either linear or bilinear in the tanglemeter coefficients, and can be interpreted as "entanglement fluxes" [21]. One notices that the coupling of the second-order terms $\beta_{i j} \sigma_{i}^{+} \sigma_{j}^{+}$to the fourth-order term $\beta_{15} \sigma_{4}^{+} \sigma_{3}^{+} \sigma_{2}^{+} \sigma_{1}^{+}$occur via the third-order terms $\beta_{7} \sigma_{3}^{+} \sigma_{2}^{+} \sigma_{1}^{+}$, $\beta_{13} \sigma_{4}^{+} \sigma_{3}^{+} \sigma_{1}^{+}, \beta_{11} \sigma_{4}^{+} \sigma_{2}^{+} \sigma_{2}^{+}, \beta_{14} \sigma_{2}^{+} \sigma_{3}^{+} \sigma_{4}^{+}$, and thus the time evolution of all $\beta_{i}$ stops when this third-order coefficients $\beta_{7}$, $\beta_{13}, \beta$, and $\beta_{14}$ vanish. Therefore, by setting the time dependence of the parameters $P_{1}^{+}, P_{2}^{+}, P_{3}^{+}$, and $P_{4}^{+}$such that they drive all four third-order coefficients to zero, we gradually reduce the $\mathrm{SU}(2)$ canonic form of Eq. (22) to the $\mathrm{SL}(2, \mathrm{C})$ form of Eq. (27). This control process results in an exponentially fast vanishing of the coefficients $\beta_{7}, \beta_{13}, \beta_{11}$, and $\beta_{14}$.

From the differential equations for these coefficients,

$$
\begin{gathered}
i \dot{\beta}_{14}=-P_{1}^{+} \beta_{15}+2 P_{2}^{+} \beta_{6} \beta_{10}+2 P_{3}^{+} \beta_{6} \beta_{12}+2 P_{4}^{+} \beta_{10} \beta_{12}, \\
i \dot{\beta}_{13}=2 P_{1}^{+} \beta_{5} \beta_{9}-P_{2}^{+} \beta_{15}+2 P_{3}^{+} \beta_{5} \beta_{12}+2 P_{4}^{+} \beta_{9} \beta_{12}, \\
i \dot{\beta}_{11}=2 P_{1}^{+} \beta_{3} \beta_{9}+2 P_{2}^{+} \beta_{3} \beta_{10}-P_{3}^{+} \beta_{15}+2 P_{4}^{+} \beta_{9} \beta_{10}, \\
i \dot{\beta}_{7}=2 P_{1}^{+} \beta_{3} \beta_{5}+2 P_{2}^{+} \beta_{3} \beta_{6}+2 P_{3}^{+} \beta_{5} \beta_{6}-P_{4}^{+} \beta_{15},
\end{gathered}
$$

we see that, in the general case, by a proper choice of the parameters $P_{i}^{+}$feedback conditions may be imposed such that these equations take the form

$$
\dot{\beta}_{7}=-\beta_{7}, \quad \dot{\beta}_{11}=-\beta_{11}, \quad \dot{\beta}_{13}=-\beta_{13}, \quad \dot{\beta}_{14}=-\beta_{14} .
$$

The evolution implied by these equations brings the nilpotential in the form of sl-tanglemeter $f_{C}$. We note that the coefficients $P_{i}^{-}$should satisfy the requirement of Eq. (64), which ensures that the nilpotential always remains in the 

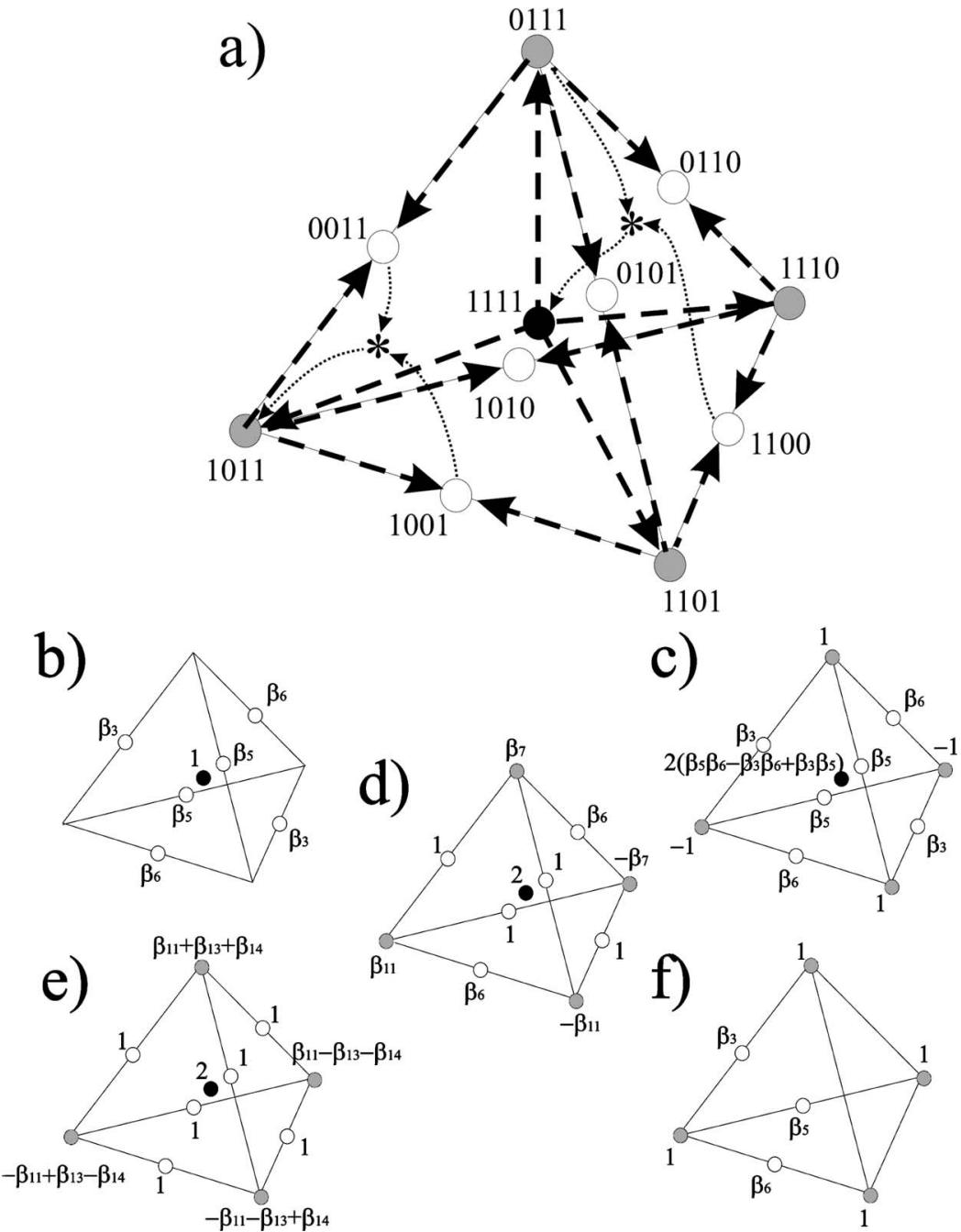

FIG. 3. Diagram illustrating the dynamics of the coefficients $\beta_{i}$ of the nilpotential $f$ for a four-qubit system subject to SL(2, C) transformations. The coefficients corresponding to quadric, cubic, and bilinear terms are shown by black, gray, and white circles, respectively. (a) Flux directions. The SL $(2, \mathrm{C})$ transformations are applied in such a way, that the polynomials remain in the canonic form with respect to $\mathrm{su}(2)$ transformations, with all linear terms $\beta_{i} \sigma_{i}^{+}=0$. The dashed arrows show the linear contributions of different states to the time derivatives of the neighboring states, and the dotted arrows jointed by asterisk depict two types of bilinear contributions. The other, similar terms may be constructed by symmetry. (b) Canonic form for the generic state and for singular classes corresponding to one (c), two (d), three (e), and four (f) vanishing eigenvalues of the determinant Eq. (69).

form of the su-tanglemeter $f_{c}$ during this evolution, even if the state does not remain in the same su-orbit. The choice of the feedback conditions is not unique, since not only Eq. (67), but any equation of the form $\dot{\beta}_{i}=-C_{i j} \beta_{j}$, with positive eigenvalues of $C_{i j}$, will cause the coefficients in front of the $(n-1)$ th order monoms to vanish. Such a choice just modifies the trajectory along which the chosen nilpotential coefficients tend to zero. We thus arrive at the tanglemeter $f_{C}$ of Eq. (27), defined up to the scaling factors. Now we can invoke the scaling of the nilpotent variables $\sigma_{i}^{+}$, and further reduce it to the sl-canonic form $f_{C}$ of Eq. (28), unless one of the bilinear coefficients vanish. The latter case corresponds to a measure-zero manifold where the canonic form may be chosen as

$$
\begin{aligned}
f_{C}= & \sigma_{2}^{+} \sigma_{1}^{+}+\beta_{5}\left(\sigma_{3}^{+} \sigma_{1}^{+}+\sigma_{4}^{+} \sigma_{2}^{+}\right)+\beta_{6}\left(\sigma_{4}^{+} \sigma_{1}^{+}+\sigma_{3}^{+} \sigma_{2}^{+}\right) \\
& +\sigma_{4}^{+} \sigma_{3}^{+} \sigma_{2}^{+} \sigma_{1}^{+},
\end{aligned}
$$

or in any equivalent form resulting from a the permutation of the indexes.

Reducing $f$ to the canonic forms of Eqs. (28)-(68) is unattainable when the determinant

$$
\left|\begin{array}{cccc}
-\beta_{15} & 2 \beta_{6} \beta_{10} & 2 \beta_{6} \beta_{12} & 2 \beta_{10} \beta_{12} \\
2 \beta_{5} \beta_{9} & -\beta_{15} & 2 \beta_{5} \beta_{12} & 2 \beta_{9} \beta_{12} \\
2 \beta_{3} \beta_{9} & 2 \beta_{3} \beta_{10} & -\beta_{15} & 2 \beta_{9} \beta_{10} \\
2 \beta_{3} \beta_{5} & 2 \beta_{3} \beta_{6} & 2 \beta_{5} \beta_{6} & -\beta_{15}
\end{array}\right|
$$

vanishes-which makes it impossible to impose the required feedback conditions, regardless of the choice of $C_{i j}$. In this 
situation, we lose the functional independence of the righthand sides of Eq. (66) that provides complete control over the dynamics of $\beta_{7}, \beta_{13}, \beta_{11}$, and $\beta_{14}$ in the generic case. In turn, this means that some linear combinations of these coefficients cannot be set to zero by any choice of $P_{i}^{+}$. In Ref. [33], a number of singular sl-entanglement classes of a fourqubit assembly have been identified. We now demonstrate how these classes arise naturally in our approach. They correspond to situations, where the system of dynamic equations (66) is degenerate and complete controllability is lost. To this end, consider the case where the determinant of Eq. (69) vanishes in more detail. This determinant is equal to zero when one or more of the eigenvalues,

$$
\begin{aligned}
& \gamma_{1}=\beta_{15}-2 \sqrt{\beta_{5} \beta_{6} \beta_{9} \beta_{10}}+2 \sqrt{\beta_{3} \beta_{6} \beta_{9} \beta_{12}}-2 \sqrt{\beta_{3} \beta_{5} \beta_{10} \beta_{12}}, \\
& \gamma_{2}=\beta_{15}+2 \sqrt{\beta_{5} \beta_{6} \beta_{9} \beta_{10}}-2 \sqrt{\beta_{3} \beta_{6} \beta_{9} \beta_{12}}-2 \sqrt{\beta_{3} \beta_{5} \beta_{10} \beta_{12}}, \\
& \gamma_{3}=\beta_{15}-2 \sqrt{\beta_{5} \beta_{6} \beta_{9} \beta_{10}}-2 \sqrt{\beta_{3} \beta_{6} \beta_{9} \beta_{12}}+2 \sqrt{\beta_{3} \beta_{5} \beta_{10} \beta_{12}}, \\
& \gamma_{4}=\beta_{15}+2 \sqrt{\beta_{5} \beta_{6} \beta_{9} \beta_{10}}+2 \sqrt{\beta_{3} \beta_{6} \beta_{9} \beta_{12}}+2 \sqrt{\beta_{3} \beta_{5} \beta_{10} \beta_{12}},
\end{aligned}
$$

vanish. We consider different cases separately.

(i) Only the first eigenvalue is zero. This implies that six coefficients in front of the bilinear terms and the coefficient $\beta_{15}$ in front of the fourth-order term are no longer independent parameters - the last one being the function of the first ones explicitly given by $\gamma_{1}=0$. The eigenvector corresponding to $\gamma_{1}$,

$$
\left(-\sqrt{\beta_{6} \beta_{10} \beta_{12}}, \sqrt{\beta_{5} \beta_{9} \beta_{12}},-\sqrt{\beta_{3} \beta_{9} \beta_{10}}, \sqrt{\beta_{3} \beta_{5} \beta_{6}}\right),
$$

gives the combination of the cubic terms that cannot be eliminated,

$$
\begin{aligned}
& -\lambda \sqrt{\beta_{6} \beta_{10} \beta_{12}} \sigma_{4}^{+} \sigma_{3}^{+} \sigma_{2}^{+}+\lambda \sqrt{\beta_{5} \beta_{9} \beta_{12}} \sigma_{4}^{+} \sigma_{3}^{+} \sigma_{1}^{+} \\
& -\lambda \sqrt{\beta_{3} \beta_{9} \beta_{10}} \sigma_{4}^{+} \sigma_{2}^{+} \sigma_{1}^{+}+\lambda \sqrt{\beta_{3} \beta_{5} \beta_{6}} \sigma_{3}^{+} \sigma_{2}^{+} \sigma_{1}^{+} .
\end{aligned}
$$

Clearly, this combination is determined up to a scaling factor $\lambda$. The nilpotential of Eq. (22) thus takes the form

$$
\begin{aligned}
f= & \beta_{3} \sigma_{2}^{+} \sigma_{1}^{+}+\beta_{5} \sigma_{3}^{+} \sigma_{1}^{+}+\beta_{9} \sigma_{4}^{+} \sigma_{1}^{+}+\beta_{6} \sigma_{3}^{+} \sigma_{2}^{+}+\beta_{10} \sigma_{4}^{+} \sigma_{2}^{+} \\
& +\beta_{12} \sigma_{4}^{+} \sigma_{3}^{+}+\lambda\left(\sqrt{\beta_{3} \beta_{5} \beta_{6}} \sigma_{3}^{+} \sigma_{2}^{+} \sigma_{1}^{+}-\sqrt{\beta_{3} \beta_{9} \beta_{10}} \sigma_{4}^{+} \sigma_{2}^{+} \sigma_{1}^{+}\right. \\
& \left.+\sqrt{\beta_{5} \beta_{9} \beta_{12}} \sigma_{4}^{+} \sigma_{3}^{+} \sigma_{1}^{+}-\sqrt{\beta_{6} \beta_{10} \beta_{12}} \sigma_{4}^{+} \sigma_{3}^{+} \sigma_{2}^{+}\right) \\
& +2\left(\sqrt{\beta_{5} \beta_{6} \beta_{9} \beta_{10}}-\sqrt{\beta_{3} \beta_{6} \beta_{9} \beta_{12}}\right. \\
& \left.+\sqrt{\beta_{3} \beta_{5} \beta_{10} \beta_{12}}\right) \sigma_{4}^{+} \sigma_{3}^{+} \sigma_{2}^{+} \sigma_{1}^{+},
\end{aligned}
$$

specified in terms of seven complex parameters $\left(\lambda, \beta_{i}\right)$. We can eliminate four of these complex parameters by setting the scaling factors of $\sigma_{i}^{+}$, and arrive at the form

$$
\begin{aligned}
f_{C}= & \beta_{3}\left(\sigma_{2}^{+} \sigma_{1}^{+}+\sigma_{4}^{+} \sigma_{3}^{+}\right)+\beta_{5}\left(\sigma_{3}^{+} \sigma_{1}^{+}+\sigma_{4}^{+} \sigma_{2}^{+}\right)+\beta_{6}\left(\sigma_{4}^{+} \sigma_{1}^{+}+\sigma_{3}^{+} \sigma_{2}^{+}\right) \\
& +\sigma_{3}^{+} \sigma_{2}^{+} \sigma_{1}^{+}-\sigma_{4}^{+} \sigma_{2}^{+} \sigma_{1}^{+}+\sigma_{4}^{+} \sigma_{3}^{+} \sigma_{1}^{+}-\sigma_{4}^{+} \sigma_{3}^{+} \sigma_{2}^{+}+2\left(\beta_{5} \beta_{6}\right. \\
& \left.-\beta_{3} \beta_{6}+\beta_{3} \beta_{5}\right) \sigma_{4}^{+} \sigma_{3}^{+} \sigma_{2}^{+} \sigma_{1}^{+},
\end{aligned}
$$

which depends only on three complex parameters. This com- bination may be considered as a class of the polynomials that cannot be reduced to the canonic forms of Eqs. (28) and (68) by the sequential application of infinitesimal transformations preserving the canonic SU(2) form. Permutation of indexes of $\sigma_{i}^{+}$give equivalent classes.

(ii) Two of the eigenvalues, say $\gamma_{1}$ and $\gamma_{2}$, in Eq. (70) are zero, that is,

$$
\beta_{15}=2 \beta_{3} \beta_{12}, \quad \beta_{5} \beta_{10}=\beta_{3} \beta_{12} .
$$

The corresponding eigenvectors,

$$
\begin{aligned}
& \left(-\sqrt{\beta_{6} \beta_{10} \beta_{12}}, \sqrt{\beta_{5} \beta_{9} \beta_{12}},-\sqrt{\beta_{3} \beta_{9} \beta_{10}}, \sqrt{\beta_{3} \beta_{5} \beta_{6}}\right), \\
& \left(-\sqrt{\beta_{6} \beta_{10} \beta_{12}},-\sqrt{\beta_{5} \beta_{9} \beta_{12}}, \sqrt{\beta_{3} \beta_{9} \beta_{10}}, \sqrt{\beta_{3} \beta_{5} \beta_{6}}\right),
\end{aligned}
$$

suggest the form of the nilpotential

$$
\begin{aligned}
f= & \beta_{3} \sigma_{2}^{+} \sigma_{1}^{+}+\beta_{5} \sigma_{3}^{+} \sigma_{1}^{+}+\beta_{9} \sigma_{4}^{+} \sigma_{1}^{+}+\beta_{6} \sigma_{3}^{+} \sigma_{2}^{+}+\beta_{10} \sigma_{4}^{+} \sigma_{2}^{+} \\
& +\beta_{12} \sigma_{4}^{+} \sigma_{3}^{+}+\lambda\left(\sqrt{\beta_{3} \beta_{5} \beta_{6}} \sigma_{3}^{+} \sigma_{2}^{+} \sigma_{1}^{+}-\sqrt{\beta_{3} \beta_{9} \beta_{10}} \sigma_{4}^{+} \sigma_{2}^{+} \sigma_{1}^{+}\right. \\
& \left.+\sqrt{\beta_{5} \beta_{9} \beta_{12}} \sigma_{4}^{+} \sigma_{3}^{+} \sigma_{1}^{+}-\sqrt{\beta_{6} \beta_{10} \beta_{12}} \sigma_{4}^{+} \sigma_{3}^{+} \sigma_{2}^{+}\right) \\
& +\mu\left(\sqrt{\beta_{3} \beta_{5} \beta_{6}} \sigma_{3}^{+} \sigma_{2}^{+} \sigma_{1}^{+}+\sqrt{\beta_{3} \beta_{9} \beta_{10}} \sigma_{4}^{+} \sigma_{2}^{+} \sigma_{1}^{+}\right. \\
& \left.-\sqrt{\beta_{5} \beta_{9} \beta_{12}} \sigma_{4}^{+} \sigma_{3}^{+} \sigma_{1}^{+}-\sqrt{\beta_{6} \beta_{10} \beta_{12}} \sigma_{4}^{+} \sigma_{3}^{+} \sigma_{2}^{+}\right) \\
& +2 \beta_{3} \beta_{12} \sigma_{4}^{+} \sigma_{3}^{+} \sigma_{2}^{+} \sigma_{1}^{+},
\end{aligned}
$$

which after a proper scaling may be simplified

$$
\begin{aligned}
f_{C}= & \sigma_{2}^{+} \sigma_{1}^{+}+\sigma_{3}^{+} \sigma_{1}^{+}+\sigma_{4}^{+} \sigma_{2}^{+}+\sigma_{4}^{+} \sigma_{3}^{+}+\beta_{6}\left(\sigma_{4}^{+} \sigma_{1}^{+}+\sigma_{3}^{+} \sigma_{2}^{+}\right) \\
& +\beta_{7}\left(\sigma_{3}^{+} \sigma_{2}^{+} \sigma_{1}^{+}-\sigma_{4}^{+} \sigma_{3}^{+} \sigma_{2}^{+}\right)+\beta_{11}\left(\sigma_{4}^{+} \sigma_{2}^{+} \sigma_{1}^{+}-\sigma_{4}^{+} \sigma_{3}^{+} \sigma_{1}^{+}\right) \\
& +2 \sigma_{4}^{+} \sigma_{3}^{+} \sigma_{2}^{+} \sigma_{1}^{+} .
\end{aligned}
$$

Again, this depends on three complex parameters and leads to equivalent classes under index permutations.

(iii) Three eigenvalues vanish, say $\gamma_{1}=\gamma_{2}=\gamma_{3}=0$. This yields

$$
\beta_{15} / 2=\sqrt{\beta_{5} \beta_{10}}=\sqrt{\beta_{3} \beta_{12}}=\sqrt{\beta_{6} \beta_{9}},
$$

which after scaling results in

$$
\begin{aligned}
f_{C}= & \sigma_{2}^{+} \sigma_{1}^{+}+\sigma_{3}^{+} \sigma_{1}^{+}+\sigma_{4}^{+} \sigma_{2}^{+}+\sigma_{4}^{+} \sigma_{3}^{+}+\sigma_{4}^{+} \sigma_{1}^{+}+\sigma_{3}^{+} \sigma_{2}^{+} \\
& +\beta_{14}\left(\sigma_{3}^{+} \sigma_{2}^{+} \sigma_{1}^{+}-\sigma_{4}^{+} \sigma_{2}^{+} \sigma_{1}^{+}+\sigma_{4}^{+} \sigma_{3}^{+} \sigma_{1}^{+}-\sigma_{4}^{+} \sigma_{3}^{+} \sigma_{2}^{+}\right) \\
& +\beta_{13}\left(\sigma_{3}^{+} \sigma_{2}^{+} \sigma_{1}^{+}+\sigma_{4}^{+} \sigma_{2}^{+} \sigma_{1}^{+}-\sigma_{4}^{+} \sigma_{3}^{+} \sigma_{1}^{+}-\sigma_{4}^{+} \sigma_{3}^{+} \sigma_{2}^{+}\right) \\
& +\beta_{11}\left(\sigma_{3}^{+} \sigma_{2}^{+} \sigma_{1}^{+}-\sigma_{4}^{+} \sigma_{2}^{+} \sigma_{1}^{+}-\sigma_{4}^{+} \sigma_{3}^{+} \sigma_{1}^{+}+\sigma_{4}^{+} \sigma_{3}^{+} \sigma_{2}^{+}\right) \\
& +2 \sigma_{4}^{+} \sigma_{3}^{+} \sigma_{2}^{+} \sigma_{1}^{+} .
\end{aligned}
$$

(iv) The last case, where all four $\gamma_{i}=0$, may be realized by setting to zero just three parameters, say $\beta_{15}=\beta_{10}=\beta_{12}=0$. This enables us to dynamically eliminate one more of the bilinear coefficients, say $\beta_{9}$, and to set all the third-order coefficients equal to one by scaling. This yields a singular canonic form,

$$
\begin{aligned}
f_{C}= & \sigma_{3}^{+} \sigma_{2}^{+} \sigma_{1}^{+}+\sigma_{4}^{+} \sigma_{3}^{+} \sigma_{1}^{+}+\sigma_{4}^{+} \sigma_{2}^{+} \sigma_{1}^{+}+\sigma_{4}^{+} \sigma_{3}^{+} \sigma_{2}^{+}+\beta_{3} \sigma_{2}^{+} \sigma_{1}^{+} \\
& +\beta_{5} \sigma_{3}^{+} \sigma_{1}^{+}+\beta_{6} \sigma_{3}^{+} \sigma_{2}^{+},
\end{aligned}
$$

which still depends on three parameters and allows permutations. This form corresponds to the situation where none of 
the four third-order terms of the nilpotential can be dynamically eliminated by local transformations, since the derivatives $\dot{\beta}_{7,11,13,14}$ are zeros regardless of the sizes of the transformation parameters $P_{i}^{ \pm}$. The only available local transformation is scaling, which allows one to set all these parameters to unity.

All five sl-tanglemeters obtained from the dynamic equations and dependent upon three complex parameters are depicted in Fig. 3. More singular classes are discussed in Appendix $\mathrm{B}$, where the relation to the singular classes of Ref. [33] is further elucidated.

\section{ENTANGLEMENT BEYOND QUBITS}

As anticipated, the nilpotent polynomials approach may be extended to describe systems more general than assemblies of qubits. Due to its increasing conceptual and practical relevance $[69,70]$, the case of qutrits is given special emphasis. With each qutrit being transformed by $\mathrm{SU}(3)$ or $\mathrm{SL}(3, \mathrm{C})$ groups, we construct appropriate nilpotent polynomials $F$ and $f=\ln F$, and thereby define the associated canonic form $F_{c}$ and tanglemeter $f_{c}$. Next, we generalize this technique to qudits.

Remarkably, the nilpotent polynomials formalism allows us to also establish contact with the framework of generalized entanglement, introduced in Refs. [58,59]. While the latter provides a notion of entanglement which relies directly on physical observables and, as such, is meaningful even in the absence of an underlying system partition (see also Ref. [71]), an important special case arises in situations where an element structure is specified, but, due to some operational or fundamental constraint, the rank $r$ of the algebra of local transformations is smaller than $d-1$, where $d$ is the element dimension. In this context, special attention is devoted to spin-1 systems, namely three-level systems restricted to evolve under the action of spin operators living in the so(3) $\equiv \operatorname{su}(2)$ subalgebra of su(3). In particular, we show how to introduce nilpotent polynomials for characterizing generalized entanglement within a single element of an assembly. In such a case, one encounters a new kind of the nilpotent variables whose squares do not vanish and only some higher powers do. We also consider entanglement among different elements of such an assembly.

We conclude by extending the nilpotent formalism to multipartite entanglement among groups of elements comprising the assembly, that is, to the case where different elements of an assembly merge, thereby creating a new assembly with elements of higher dimension.

\section{A. Qutrits and qudits}

In order to describe qudit entanglement, one needs to invoke the Lie algebras of higher rank su(d) and their complex versions $\operatorname{sl}(d, \mathrm{C})$. The construction of nilpotent polynomials for such systems is based on the so-called Cartan-Weyl decomposition. We illustrate this for qutrits, $d=3$, and then generalize to arbitrary $d$.

\section{Nilpotent variables for qutrits}

Let us start by reminding some basic facts about group theory and Lie algebra representation theory $[72,73]$. The $d^{2}-1$ generators of the algebra $\mathrm{sl}(d)$, the complexified $\operatorname{su}(d)$, may be decomposed into three sets.

(i) A set $\mathcal{H}$ of $r=d-1$ linearly independent, mutually commuting generators of a Cartan subalgebra $\operatorname{span}(\mathcal{H})[r$ is the rank of the algebra and $\operatorname{span}(\mathcal{H})$ is the vector space spanned by $\mathcal{H}$ ]. In a faithful matrix representation, the most natural choice for Cartan generators are traceless $d \times d$ diagonal matrices.

(ii) A set $\{\mathcal{E}\}$ of $d(d-1) / 2$ "raising" generators spanning a nilpotent subalgebra $\operatorname{span}(\mathcal{E}) \subset \operatorname{su}(d)$. The elements of $\{\mathcal{E}\}$ and of $\operatorname{span}(\mathcal{E})$ can be represented by the matrices with nonzero elements only above the main diagonal.

(iii) $d(d-1) / 2$ Hermitian conjugate "lowering" generators spanning a nilpotent subalgebra $\operatorname{span}\{\mathcal{F}\}$ represented by matrices with nonzero elements only below the diagonal.

In the case of su(2), each of the above sets contains a single generator: $\mathcal{H}=\left\{\sigma^{3}=\sigma_{z}\right\}, \mathcal{E}=\left\{\sigma^{+}\right\}$, and $\mathcal{F}=\left\{\sigma^{-}\right\}$. For $\mathrm{su}(3)$ having eight generators represented by the eight GellMann $\lambda^{a}$ matrices [72], the Cartan subalgebra involves two generators usually chosen as

$$
\lambda^{3}=\left(\begin{array}{ccc}
1 & 0 & 0 \\
0 & -1 & 0 \\
0 & 0 & 0
\end{array}\right), \quad \lambda^{8}=\frac{1}{\sqrt{3}}\left(\begin{array}{ccc}
1 & 0 & 0 \\
0 & 1 & 0 \\
0 & 0 & -2
\end{array}\right) .
$$

The basis $\mathcal{E}$ for the raising nilpotent subalgebra is comprised of three elements,

$$
\begin{aligned}
& s^{+}=\frac{\lambda^{1}+i \lambda^{2}}{2}=\left(\begin{array}{lll}
0 & 1 & 0 \\
0 & 0 & 0 \\
0 & 0 & 0
\end{array}\right), \\
& u^{+}=\frac{\lambda^{4}+i \lambda^{5}}{2}=\left(\begin{array}{lll}
0 & 0 & 1 \\
0 & 0 & 0 \\
0 & 0 & 0
\end{array}\right), \\
& t^{+}=\frac{\lambda^{6}+i \lambda^{7}}{2}=\left(\begin{array}{lll}
0 & 0 & 0 \\
0 & 0 & 1 \\
0 & 0 & 0
\end{array}\right),
\end{aligned}
$$

while $\mathcal{F}$ includes their Hermitian conjugates.

The elements of $\mathcal{E}$ and $\mathcal{F}$ are the root vectors of su(3). It means that for $e \in \mathcal{E}, f \in \mathcal{F}$, and $h \in \operatorname{span}(\mathcal{H})$, the commutator $[e, h]$ is proportional to $e$, and the commutator $[f, h]$ is proportional to $f$. The subalgebra $\mathcal{E}$ is nilpotent, meaning that multiple commutators $\left[e_{1}, \cdots,\left[e_{p-1}, e_{p}\right] \cdots\right]$ vanish starting at some level $p-1$. For su(3) with only one nontrivial commutator $\left[s^{+}, t^{+}\right]=u^{+}$, double commutators already vanish. For $\mathrm{su}(d)$, they vanish at the level $d-1$, coinciding with the rank of the algebra $r$.

A generic pure state of a qutrit may be represented as

$$
\left.|\psi\rangle=\psi_{0}|0\rangle+\psi_{1}|1\rangle+\psi_{2}\left|2=\left(\psi_{0}+\psi_{1} t^{+}+\psi_{2} u^{+}\right)\right| 0\right\rangle,
$$

with 


$$
|0\rangle \equiv\left(\begin{array}{l}
0 \\
0 \\
1
\end{array}\right), \quad|1\rangle \equiv\left(\begin{array}{l}
0 \\
1 \\
0
\end{array}\right), \quad|2\rangle \equiv\left(\begin{array}{l}
1 \\
0 \\
0
\end{array}\right) .
$$

Equation (80) generalizes a similar representation for the qubit pure states extensively discussed so far. The operators $t^{+}, u^{+}$belong to $\mathcal{E}$ and commute. Note that even for larger $d$, such a set of $d-1=r$ commuting nilpotent operators always exists [74], allowing one to express a generic qudit state as a first-order polynomial of commuting nilpotent variables.

The state $|0\rangle$, which is annihilated by all "lowering" generators of $\mathcal{F}$, plays the special role of the Fock vacuum or reference state. But the choice Eq. (81) is not unique. Actually, any qutrit state can serve as a valid reference state. For each $|\widetilde{0}\rangle=U|0\rangle, U \in \mathrm{SU}(3)$, the analysis above applies if one merely changes (conjugates) accordingly the Cartan-Weyl decomposition and defines

$$
\widetilde{\mathcal{H}}=U \mathcal{H} U^{-1}, \quad \widetilde{\mathcal{E}}=U \mathcal{E} U^{-1}, \quad \widetilde{\mathcal{F}}=U \mathcal{F} U^{-1}
$$

$\operatorname{Again}, \operatorname{span}(\widetilde{\mathcal{E}})$ and $\operatorname{span}(\widetilde{\mathcal{F}})$ are nilpotent; again, $|\widetilde{0}\rangle$ are annihilated by the elements of $\widetilde{\mathcal{F}}$, etc. For example, if the choice $|\widetilde{0}\rangle=|1\rangle$ is made, the elements of $\mathcal{E}$ are $\widetilde{s}^{+}=-u^{+}, \widetilde{t}^{+}=$ $-t^{-}, \widetilde{u}^{+}=s^{+}$. In this case, a generic qutrit state would be expressed as

$$
|\psi\rangle=\left(-\psi_{0} \tilde{t}^{+}+\psi_{1}+\psi_{2} \tilde{u}^{+}\right)|1\rangle=\left(\psi_{0} t^{-}+\psi_{1}+\psi_{2} s^{+}\right)|1\rangle .
$$

The choice of Eq. (80) is more natural for discussing qutrits, whereas the choice made in Eq. (82) allows one to associate $\lambda^{3}$ with the spin projection operator $S_{z}$ for a spin-1 system, with the vacuum state $|\widetilde{0}\rangle=|1\rangle$ naturally corresponding to the lowest eigenvalue -1 of this operator. The state $|1\rangle$ is socalled extremal weight state for the representation and is discussed in Sec. IV C in more details. In any case, this choice is mainly a matter of state labeling, which in most cases is dictated by convenience and can then be done accordingly for each particular physical problem.

In analogy with Eq. (13), the state of $n$ qutrits may then be written as

$$
\begin{aligned}
|\Psi\rangle & =\sum_{\left\{\nu_{i}, \eta_{i}=0,1 / \nu_{i} \eta_{i}=0\right\}} \psi_{2 v_{n}+\eta_{n}, \ldots, 2 \nu_{1}+\eta_{1}} \prod_{i=1}^{n}\left(u_{i}^{+}\right)^{\nu_{i}}\left(t_{i}^{+}\right)^{\eta_{i}|\mathrm{O}\rangle} \\
& =F\left(u_{i}^{+}, t_{i}^{+}\right)|\mathrm{O}\rangle
\end{aligned}
$$

where $|\mathrm{O}\rangle=|0, \ldots, 0\rangle$ denotes the reference state. Our next steps are (i) to consider the nilpotential $f=\ln F$; (ii) to bring it into the canonic form specified by the requirement of maximum population of the state $|\mathrm{O}\rangle$, followed by the requirement of maximum population of the state $|1\rangle$ $=|1, \ldots, 1\rangle$, as discussed at the end of Sec. II A; and (iii) to normalize $\left|\Psi_{C}\right\rangle$ by the condition $\left\langle\mathrm{O} \mid \Psi_{C}\right\rangle=1$. The tangle meter $f_{C}$ thereby obtained provides one with simple entanglement characteristics and relevant insights.

\section{Entanglement of two qutrits}

We select $|\mathrm{O}\rangle=|0,0\rangle$ as the reference state. The relevant nilpotent variables are $u_{i}^{+}$and $t_{i}^{+}$, where the index $i=1,2$ labels the qutrits. An arbitrary assembly state may then be written as

$$
\begin{aligned}
|\Psi\rangle= & F\left(u_{i}^{+}, t_{i}^{+}\right)|\mathrm{O}\rangle=\left(\psi_{00}+\psi_{01} t_{1}^{+}+\psi_{02} u_{1}^{+}+\psi_{10} t_{2}^{+}+\psi_{20} u_{2}^{+}\right. \\
& \left.+\psi_{11} t_{1}^{+} t_{2}^{+}+\psi_{12} t_{2}^{+} u_{1}^{+}+\psi_{21} u_{2}^{+} t_{1}^{+}+\psi_{22} u_{2}^{+} u_{1}^{+}\right)|\mathrm{O}\rangle
\end{aligned}
$$

The requirement of maximum population of $|\mathrm{O}\rangle$ implies that the linear terms in $F\left(u_{i}^{+}, t_{i}^{+}\right)$vanish. This is proved by inspecting the variation of the population under local unitary transformations, similarly to the qubit case [cf. Eq. (8)]. Imposing our normalization constraint, dictating that the expansion of $F$ starts with 1, we get

$$
F_{c}=1+\alpha_{11} t_{2}^{+} t_{1}^{+}+\alpha_{12} t_{2}^{+} u_{1}^{+}+\alpha_{21} u_{2}^{+} t_{1}^{+}+\alpha_{22} u_{2}^{+} u_{1}^{+} .
$$

Thus, we are left with the reference state and four excited states. The form in Eq. (85) is invariant with respect to the subgroup $\mathrm{SU}_{2}(2) \otimes \mathrm{SU}_{1}(2)$ of local transformations which can mix the levels $|1\rangle$ and $|2\rangle$ for each qutrit, but preserve the population of the state $|\mathrm{O}\rangle$. Using this freedom, we further restrict the canonic form by acting on $|\Psi\rangle$ with the transformations of $\mathrm{SU}_{2}(2) \otimes \mathrm{SU}_{1}(2)$ that maximizes the population of the state $|11\rangle=t_{2}^{+} t_{1}^{+}|0\rangle$, while the population of the reference state $|O\rangle$ is already maximized. This eliminates the terms $\propto t_{2}^{+} u_{1}^{+}$and $\propto u_{2}^{+} t_{1}^{+}$in $F_{c}$. Thus, we finally obtain

$$
F_{c}\left(u_{2}^{+}, t_{2}^{+}, u_{1}^{+}, t_{1}^{+}\right)=1+\alpha_{11} t_{2}^{+} t_{1}^{+}+\alpha_{22} u_{2}^{+} u_{1}^{+} .
$$

The polynomial $F_{c}$ depends only on two complex parameters $\alpha_{i i}$, which can be set real and positive by a local phase transformation,

$$
\exp \left(i \gamma_{2} \lambda_{2}^{3}+i \delta_{2} \lambda_{2}^{8}+i \gamma_{1} \lambda_{1}^{3}+i \delta_{1} \lambda_{1}^{8}\right) .
$$

Thus, two real parameters suffice for characterizing entanglement between two qutrits, as depicted in Fig. 1(b). This is consistent with the result given by a straightforward application of the bipartite Schmidt decomposition.

When counting the number of invariants $D_{\text {su }}$ for a twoqutrit state with the help of the expression

$$
D_{\text {su }}=2 \times 3^{n}-8 n-2,
$$

we find $D_{\text {su }}=0$ for $n=2$. As for the two-qubit case, this number differs from the actual number of independent parameters because the phase transformation of Eq. (87) has more parameters than the number of the coefficients in Eq. (85), and some of them act on the coefficients in the same way [cf. Eq. (10) and the discussion thereabout]. The counting given by Eq. (88) holds for $n \geqslant 3$.

\section{Entanglement in a generic qudit assembly}

The above analysis suggests the following generalization to an assembly of $n$ qudits. Let $d_{i}$ denote the local dimension of the $i$ th element with the associated $\operatorname{su}\left(d_{i}\right)$ algebra. For each element $i$, we choose a reference state $|0\rangle_{i}$ and perform the corresponding Cartan-Weyl decomposition $\mathcal{H}_{i} \oplus \mathcal{E}_{i} \oplus \mathcal{F}_{i}$ for the generators of this algebra, in such a way that $f|0\rangle_{i}$ $=0$ for all $f \in \mathcal{F}_{i}$. The most convenient choice for $|0\rangle_{i}$ is the 
state with only the lowest level occupied. One may choose a basis in the qudit Hilbert space involving the vacuum state $|0\rangle_{i}$ and the "excited states," such that each basis state represents a joint eigenstate of all generators $\lambda_{i}^{\kappa_{i}}$ in the Cartan subalgebra $\left(\kappa_{i}=1, \cdots, d_{i}-1\right)$. The eigenvalues of $\left\{\lambda_{i}^{\kappa_{i}}\right\}$ thus provide good quantum numbers labeling the qudit state.

Next, we choose a set of commuting nilpotent generators $\left\{\nu_{i}^{\kappa_{i}}\right\} \subseteq \mathcal{E}_{i}$ that may be employed as nilpotent variables. To be specific, let us enumerate these variables such that $|1\rangle_{i}$ $=\nu_{i}^{1}|0\rangle_{i}$ corresponds to the first excited state of $i$ th element, $|2\rangle_{i}=\nu_{i}^{2}|0\rangle_{i}$ to the second, etc. It is convenient to add the value 0 to the allowed range of $\kappa_{i}$ with the convention $\nu_{i}^{0}$ $=1$. In other words, the indexes are defined by the condition $\nu_{i}^{\kappa_{i}}\left|0_{i} k_{i}\right\rangle$. A polynomial $F\left\{\nu_{i}^{\kappa_{i}}\right\}$ of these nilpotent arguments characterizes a generic quantum state of the assembly as the latter may be obtained by acting by the operator $F$ on the corresponding assembly reference state $|\mathrm{O}\rangle=\Pi_{i}|0\rangle_{i}$.

In analogy with the procedure for two qutrits described in Sec. IV A 2, we can, using local $\mathrm{SU}\left(d_{i}\right)$ operations, maximize the population of the vacuum state and eliminate the terms linear in the nilpotent operators. The function $F$ acquires the form generalizing Eq. (85),

$$
F=\alpha_{\left\{\kappa_{i}\right\}} \prod_{i=1}^{n} \nu_{i}^{\kappa_{i}},
$$

where repeated indexes imply summation, $n$ is the number of assembly elements, and $\Sigma_{i} \kappa_{i} \neq 1$. The state is normalized to unit amplitude of the reference state, $\alpha_{\{0 \cdots 0\}}=1$, as earlier. As a next step, we maximize the population of the symmetric state $|1, \ldots, 1\rangle$ using the transformations of the subgroup $\otimes_{i} \mathrm{SU}\left(d_{i}-1\right)$, where the tensor product is taken over all $i$ with $d_{i}>2$, which preserve the reference state. At the third step, we maximize the population of the state $\left|\left\{k_{i}\right\}\right\rangle$, where $k_{i}=2$ for the elements with $d_{i}>2$ while for qubits the label $k_{i}$ is "frozen" at the value $k_{i}=1$, and maximization is done using the transformations of the subgroup $\otimes_{i} \mathrm{SU}\left(d_{i}-2\right)$ that affect neither the reference state nor the first excited state, and the tensor product involves now the elements with $d_{i}>3$. If the assembly involves five-level elements, we are allowed at the next step to maximize the population of the state $\left|\left\{k_{i}\right\}\right\rangle$, where $k_{i}=1$ for qubits, $k_{i}=2$ for qutrits and $k_{i}=3$ for the elements with $d_{i}>3$, etc.

For example, for an assembly of a five-level system, a four-level system, and a qutrit, one should consecutively maximize (see also Fig. 4):

(i) the population of the state $|0,0,0\rangle$ by the transformations from $\mathrm{SU}(5) \otimes \mathrm{SU}(4) \otimes \mathrm{SU}(3)$;

(ii) the population of the state $|1,1,1\rangle$ by the transformations from $\mathrm{SU}(4) \otimes \mathrm{SU}(3) \otimes \mathrm{SU}(2)$;

(iii) the population of the state $|2,2,2\rangle$ by the transformations from $\mathrm{SU}(3) \otimes \mathrm{SU}(2)$;

(iv) the population of the state $|3,3,2\rangle$ by the transformations from the remaining $\mathrm{SU}(2)$ mixing the threedimensional and the 4th excited states of the five-level system.

This procedure eventually reduces the nilpotent polynomial $F$ to the canonic form of Eq. (85), where some coeffi-
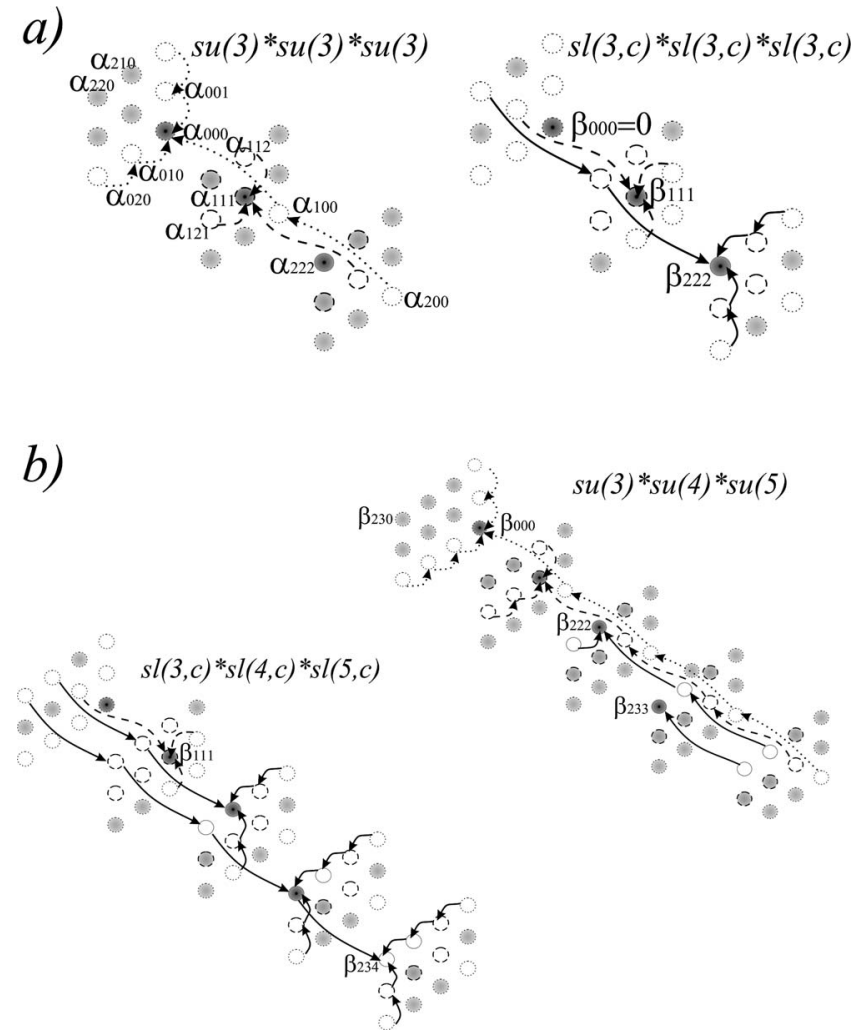

FIG. 4. (a) A possible strategy for identifying the tanglemeter $f_{c}$ for three qutrits. The choice of the parameters $P_{i}^{\kappa}$ of local unitary transformations is done in such a way that the transformations sequentially eliminate the terms in $F_{c}$ that are coupled to the states $|k, k, k\rangle$ by just one local operator. The $\mathrm{SU}(3)$ transformations maximize the population of the vacuum $|\mathrm{O}\rangle=|0,0,0\rangle$. Next, the $\mathrm{SU}(2)$ transformations not acting on the states $\left|0_{i}\right\rangle$ shown by dotted circles, and only the states shown by dashed and solid circle are mixed, maximize the population of the state $|1,1,1\rangle$. The population of the state $|2,2,2\rangle$ cannot be further maximized by local unitary operations. However, SL(3) and SL(2) dynamic transformations can eliminate some of the coefficients $\beta$ directly connected to such states. (b) The same diagrams for the case of elements with different dimensions. The arrows show the tanglemeter coefficients that "absorb" the coefficients vanishing in the course of the controlled dynamics.

cients $\alpha_{\left\{\kappa_{i}\right\}}$ now vanish. In order to better understand the pattern, consider two examples: an assembly of three qutrits, and of two qutrits and a qubit. In the first case, the canonic form is

$$
\begin{aligned}
F_{c}= & 1+\alpha_{011} t_{2}^{+} t_{1}^{+}+\alpha_{012} t_{2}^{+} u_{1}^{+}+\alpha_{021} u_{2}^{+} t_{1}^{+}+\alpha_{022} u_{2}^{+} u_{1}^{+}+\alpha_{101} t_{3}^{+} t_{1}^{+} \\
& +\alpha_{102} t_{3}^{+} u_{1}^{+}+\alpha_{201} u_{3}^{+} t_{1}^{+}+\alpha_{202} u_{3}^{+} u_{1}^{+}+\alpha_{110} t_{3}^{+} t_{2}^{+}+\alpha_{120} t_{3}^{+} u_{2}^{+} \\
& +\alpha_{210} u_{3}^{+} t_{2}^{+}+\alpha_{220} u_{3}^{+} u_{2}^{+}+\alpha_{111} t_{3}^{+} t_{2}^{+} t_{1}^{+}+\alpha_{122} t_{3}^{+} u_{2}^{+} u_{1}^{+} \\
& +\alpha_{212} u_{3}^{+} t_{2}^{+} u_{1}^{+}+\alpha_{221} u_{3}^{+} u_{2}^{+} t_{1}^{+}+\alpha_{222} u_{3}^{+} u_{2}^{+} u_{1}^{+},
\end{aligned}
$$

whereas for two qutrits and a qubit (labeled by the index 3), it looks as follows: 


$$
\begin{aligned}
F_{c}= & 1+\alpha_{4} t_{2}^{+} t_{1}^{+}+\alpha_{5} t_{2}^{+} u_{1}^{+}+\alpha_{7} u_{2}^{+} t_{1}^{+}+\alpha_{8} u_{2}^{+} u_{1}^{+}+\alpha_{10} t_{3}^{+} t_{1}^{+} \\
& +\alpha_{11} t_{3}^{+} u_{1}^{+}+\alpha_{12} t_{3}^{+} t_{2}^{+}+\alpha_{15} t_{3}^{+} u_{2}^{+}+\alpha_{13} t_{3}^{+} t_{2}^{+} t_{1}^{+}+\alpha_{17} t_{3}^{+} u_{2}^{+} u_{1}^{+},
\end{aligned}
$$

with the identification $\nu_{i}^{1}=t_{i}^{+}, \nu_{i}^{2}=u_{i}^{+}$. In Eq. (90), the indexes of $\alpha$ label the individual qutrit states, while in Eq. (91) the notation relies on the decimal representation of the base- 3 numbers associated with these indexes, $112 \rightarrow 14$, etc. The canonic forms are defined, as before, up to phase factors of the nilpotent variables $\nu_{j}^{\kappa_{j}}$.

The forms of Eqs. (90) and (91) do not involve linear terms. Neither do they involve any term proportional to $t_{3}^{+} t_{2}^{+} u_{1}^{+}, t_{3}^{+} u_{2}^{+} t_{1}^{+}$, and (for three qutrits) $u_{3}^{+} t_{2}^{+} t_{1}^{+}$. In other words, the amplitudes of the states $|1,1,2\rangle,|1,2,1\rangle$ and, for three qutrits, $|2,1,1\rangle$ vanish. This vanishing is achieved at the second stage of our procedure when the population of the state $|1,1,1\rangle$ is maximized by the transformations from $\otimes_{i=1}^{3} \mathrm{SU}(2)_{i}$ for three qutrits, and from $\mathrm{SU}_{2}(2) \otimes \mathrm{SU}_{1}(2)$ for two quadrats and a qubit. Indeed, from the viewpoint of the remaining $\mathrm{SU}(2)$ transformations, the state $|1\rangle$ may be regarded as a reference state, and amplitudes like $|1,1,2|$ vanish for the same reason why the amplitudes $|1,0, \ldots, 0\rangle$ vanished when the population of $|O\rangle$ were maximized. It is clear that, e.g., for an assembly of three four-level systems, where the procedure involves three steps, the canonic state has vanishing coefficients of the basis states $|2,1,1\rangle,|1,2,1\rangle,|1,1,2\rangle$, $|3,1,1\rangle,|1,3,1\rangle,|1,1,3\rangle,|3,2,2\rangle,|2,3,2\rangle$ and $|2,2,3\rangle$. Generally, if the procedure involves the maximization of the population of the state $\left|\left\{k_{i}\right\}\right\rangle$, the vanishing amplitudes are $\left|\left\{k_{i}^{\prime}\right\}\right\rangle$, where the sets $\left\{k_{i}\right\}$ and $\left\{k_{i}^{\prime}\right\}$ differ only in one position $i_{0}$ and $k_{i_{0}}^{\prime}$ $>k_{i_{0}}$.

One can give a general definition of the canonic state which applies to the case of an assembly with elements of different dimensions by marking the element states by a formal index $\mathbf{k}_{i}$ which takes the value $\mathbf{k}_{i}=\mathbf{k}_{i}$ for $\mathbf{k}_{i} \leqslant \mathbf{d}_{i}$ and $\mathbf{k}_{i}$ $=\mathbf{d}_{i}$ for $\mathbf{k}_{i}>\mathbf{d}_{i}$. For example, for a qubit, the state $|\mathbf{0}\rangle$ with $\mathbf{k}=\mathbf{0}$ is the vacuum state $|0\rangle$, the state $|\mathbf{1}\rangle$ is the excited state $|1\rangle$ of the qubit, while $|\mathbf{2}\rangle=|\mathbf{3}\rangle=\cdots=|1\rangle$. For a qutrit $|\mathbf{0}\rangle$ $=|0\rangle,|\mathbf{1}\rangle=|1\rangle,|\mathbf{2}\rangle=|2\rangle$, and $|\mathbf{3}\rangle=|\mathbf{4}\rangle=\cdots=|2\rangle$. The su-canonic state corresponds to maximization of the populations of the states $|\mathbf{k}, \ldots, \mathbf{k}, \ldots, \mathbf{k}\rangle$ with identical formal index $\mathbf{k}$ $=\mathbf{0}, 1, \ldots, d_{\max }$, while the states $\left|\mathbf{k}_{1}, \ldots, \mathbf{k}_{i}, \ldots, \mathbf{k}_{n}\right\rangle$ with $\mathbf{k}_{i}$ $\geqslant \mathbf{k}$ are eliminated.

Going back to Eq. (90), we observe that the canonic polynomial depends in this case on 17 complex parameters, six of which can be set real by a proper choice of the phase factors of the nilpotent variables $u_{i}^{+}$and $t_{i}^{+}$. This coincides with the number $D_{\mathrm{su}}=28$ suggested by the formula given in Eq. (88) valid for $n>2$.

The coefficients $\alpha_{\left\{\kappa_{i}\right\}}$ can be treated as orbit markers characterizing the qudit entanglement. But the coefficients of the tanglemeter $f_{c}=\ln F_{c}$ provide, as we already saw for qubits, a more direct and physical description. In particular, the criterion of Eq. (18), indicating whether two groups, $A$ and $B$, of a bipartition are entangled, may be straightforwardly generalized. We have

$$
\frac{\partial^{2} f\left(\left\{\nu_{i}^{\kappa_{i}}\right\}\right)}{\partial \nu_{i}^{\kappa_{i}} \partial \nu_{m}^{\kappa_{m}}}=0, \quad i \in A, \quad m \in B .
$$

As before, this criterion holds for a noncanonic $f$.
The construction of the sl-tanglemeter may be accomplished by analogy to the qubit case. One can derive and directly employ the dynamic equations for $f$, and by a proper choice of the transformation parameters $P_{i}^{\kappa}$ eliminate the coefficients $\beta_{\left\{\kappa_{i}\right\}}$ corresponding to the states adjacent to $|\mathbf{k}, \ldots, \mathbf{k}\rangle$, that are the states $\left|\mathbf{k}, \ldots, \mathbf{k}^{\prime} \neq \mathbf{k}, \ldots, \mathbf{k}\right\rangle$ different from the state $|\mathbf{k}, \ldots, \mathbf{k}\rangle$ only in a single position. In other words, the coefficients $\beta_{\left\{\kappa_{i}\right\}}$ to be eliminated contain the indexes $\left\{\kappa_{i}\right\}=\kappa_{1}, \ldots, \kappa_{n}$ that correspond to the most symmetric state $|\mathbf{k}, \ldots, \mathbf{k}\rangle$ in all but one position. Thereby one gets

$$
f_{C}=\beta_{\left\{\kappa_{i}\right\}} \prod_{i=1}^{n} \nu_{i}^{\kappa_{i}},
$$

where many of the coefficients $\beta_{\left\{\kappa_{i}\right\}}$ vanish. Indeed, as SL transformations have more parameters than SU transformations, we can now bring to zero more coefficients than in the SU case. Namely, the sum on the right-hand side of Eq. (92) contains no terms corresponding to the states directly coupled to $|\mathbf{k}, \ldots, \mathbf{k}\rangle$ by any single local transformation, not only those involving "higher" states with $k^{\prime} \geqslant k$.

Without explicitly presenting the corresponding dynamic equations, we illustrate this idea in Fig. 4 for the case of (a) three qutrits and for the case (b) an assembly comprising three different elements with dimensions $d_{1}=3, d_{2}=4$, and $d_{3}=5$. For qutrits, nine coefficients $\beta_{001}, \beta_{010}, \beta_{100}, \beta_{002} \beta_{020}$, $\beta_{200}, \beta_{112} \beta_{211}$, and $\beta_{121}$ of the nilpotential vanish, in accordance with Eq. (90), and give the tanglemeter in the su case. For the sl-tanglemeter, nine other coefficients $\beta_{011} \beta_{110}, \beta_{101}$, $\beta_{202} \beta_{220}, \beta_{022}$, and $\beta_{122} \beta_{212}, \beta_{221}$ can be set to zero as well. As one sees, we are left with $\beta_{012} \beta_{201}, \beta_{120}, \beta_{102} \beta_{210}, \beta_{021}$, $\beta_{111}$, and $\beta_{222}$, that is the generic $\operatorname{SL}(3, \mathrm{C})$ canonic form of $f$ contains these eight complex parameters, while six of these parameters can be specified by a proper choice of the scaling factors. One thus obtains

$$
\begin{aligned}
f_{C}= & \beta_{g}\left(u_{3}^{+} t_{2}^{+}+u_{2}^{+} t_{1}^{+}+t_{3}^{+} u_{1}^{+}\right)+t_{3}^{+} t_{2}^{+} t_{1}^{+}+\beta_{u}\left(t_{2}^{+} u_{1}^{+}+u_{3}^{+} t_{1}^{+}+t_{3}^{+} u_{2}^{+}\right) \\
& +u_{3}^{+} u_{2}^{+} u_{1}^{+} .
\end{aligned}
$$

In other words, the sl-tanglemeter is characterized in this case by two complex parameters $\beta_{g}$ and $\beta_{u}$-consistent with the counting $D=2\left(3^{3}-3 \times 8-1\right)=4$ of the coset dimension.

In case (b), the maximum symmetric states $|\mathbf{k}, \mathbf{k}, \mathbf{k}\rangle$, with $\mathbf{k}=0,1,2,3,4$, are the states $|0,0,0\rangle,|1,1,1\rangle,|2,2,2\rangle,|2,3,3\rangle$, and $|2,3,4\rangle$, respectively. The tanglemeter coefficients vanish when in one of the positions the index takes a larger value. These coefficients are $\beta_{00 k}$, with $k=1,2,3,4, \beta_{0 k 0}$, with $k$ $=1,2,3$ and $\beta_{k 00}$ with $k=1,2$ along with the coefficients $\beta_{112}, \beta_{113}, \beta_{114}, \beta_{223}, \beta_{224}, \beta_{334}, \beta_{121}, \beta_{131}, \beta_{232}$, and $\beta_{211}$. For the sl-tanglemeter this list is complimented by the coefficients that have one of the indexes smaller. These are the coefficients $\beta_{011}, \beta_{101}, \beta_{110}$, the coefficients $\beta_{k 22}, \beta_{2 k 2}, \beta_{22 k}$, with $k=0,1$, and the coefficients $\beta_{133}, \beta_{033}, \beta_{213}, \beta_{203}, \beta_{231}$, $\beta_{230}, \beta_{134}, \beta_{034}, \beta_{214}, \beta_{204}, \beta_{231}$, and $\beta_{230}$. Note that the coefficient $\beta_{233}$ is not in this list, since though its indexes differ from the indexes $\{\mathbf{4}, \mathbf{4}, \mathbf{4}\}$ of the state $|2,3,4\rangle$ in one position, this coefficient corresponds to the symmetric state $|\mathbf{3}, \mathbf{3}, \mathbf{3}\rangle=|2,3,3\rangle$ and hence cannot be elliminated. We are therefore left with 21 complex coefficients that can be scaled 
by choosing of nine complex factors in front of nilpotent variables. Thus the sl-entanglement is characterized by 24 real parameters, which is again consistent with the direct counting of the coset dimension,

$$
D=2\left[d_{1} d_{2} d_{3}-\left(d_{1}^{2}-1\right)-\left(d_{2}^{2}-1\right)-\left(d_{3}^{2}-1\right)-1\right] .
$$

\section{B. Generalized entanglement and generating functions}

We now consider a situation where the local operations available for qudits are restricted by some operational or fundamental constraint, so that they cannot ensure universal local transformations. In particular, we focus on the case where the restricted local operations form a subgroup $\mathrm{SU}(m) \subset \mathrm{SU}(d)$, with $m<d$, of the full unitary transformation set. As mentioned, this is a special relevant instance of a more general, subsystem-independent entanglement setting (generalized entanglement) formalized in Refs. [58,59]. One of the main implications of the latter approach is, in turn, to point to an intimate connection between entanglement and so-called generalized coherent states (GCSs) [75,76], which is also useful to place our current analysis in a broader context.

GCSs may be constructed for quantum systems described by a dynamic-symmetry Lie group, which typically is assumed to be reductive or semisimple: a family of GCSs may be thought of as an orbit resulting from application of the Lie group to a reference state, which is identified with an extremal-weight state in the irreducible representation of the underlying dynamical Lie algebra. Within this general setting, the canonical coherent states of a quantum harmonicoscillator [77] may be recovered as resulting from the application of the displacement operator $\left[\exp \left(a z^{*}-a^{\dagger} z\right)\right]$ to the ground state $|0\rangle$. Here, as usual, $a$ and $a^{\dagger}$ denote annihilation and creation operators, respectively, and $z=x+i p \in \mathrm{C}$ stands for displacement in the phase space $(x, p)$. The relevant dynamic groups are generated by the Heisenberg-Weyl algebras, either $\mathfrak{h}_{3}=\left\{\mathbb{I}, a, a^{\dagger}\right\}$ that allows for arbitrary displacements, or $\mathfrak{h}_{4}=\left\{\mathbb{I}, a, a^{\dagger}, a^{\dagger} a\right\}$ that in addition involves the phase space rotation $\exp \left(i \varphi a^{\dagger} a\right)$ by an angle $\varphi$ [78]. Physically, the most representative manifestation of the coherent states is the fact that external interventions realized via $a \dagger$ and $a$ cannot change the structure of the wave function expressed in terms of the Wigner function, but just displace and rotate it in the phase space. One can also include the operation $\left\{\exp i\left[s\left(a^{\dagger}\right)^{2}+s^{*} a^{2}\right]\right\}$ of squeezing $s \in \mathrm{C}$ by remaining within a finite-dimensional (so-called "two-photon") dynamic algebra. Clearly, the algebra describing arbitrary transformation on the oscillator Hilbert space is infinite dimensional, and destroys the manifestations typical of coherent states.

Interestingly, the mathematical techniques employed to describe GCSs are extremely close to the ones we use for nilpotent polynomials: any GCS is constructed from a minimum-weight reference state of a semisimple Lie algebra by application of a function of the appropriate Cartan-Weyl raising operators which, for a finite-dimensional Hilbert space, are indeed nilpotent. One can always construct GCSs of a qudit, relative to its full su(d) algebra of transformations.
The resulting family is, of course, identical with the set of possible pure states of the system. For a qudit assembly with $n$ elements, it was shown in Ref. [58] that conventional unentangled states are identical with the set of GCSs under arbitrary local transformations, that is, with respect to the algebra $\oplus_{i=1}^{n} \operatorname{su}\left(d_{i}\right)$, with rank $R=\sum_{i}\left(d_{i}-1\right)$. Geometrically, such GCSs are extremal (in the convex sense) in the set of states which may specified only through expectation values of arbitrary local observables. However, such extremality property may no longer be fulfilled upon restricting the transformations on each element to a proper subalgebra, $m_{i}<d_{i}$-implying the possibility of generalized entanglement relative to the restricted observable set. Here, we further develop the connection between entanglement and GCSs in terms of the nilpotent polynomials approach. In a way, we can say that we consider entanglement related to GCSs both within each element as well as entanglement among GCSs of different elements of the assembly, and construct in each case appropriate characteristics based on nilpotent variables.

The first step toward accomplishing the above goal is to obtain a proper description of the group of restricted local transformations, by embedding it as a subgroup into the fullrank $r_{i}=d_{i}-1$ group of local transformations. In other words, among the generators of full group we must specify the linear combinations that correspond to the generators of the restricted local transformations. To this end, it turns out that so-called generating function technique offers a convenient tool. Let $|\Psi\rangle$ be a state of the assembly normalized to the unit amplitude of the vacuum state, and apply the operator $\exp \left[\sum_{i} x_{i}^{\kappa_{i}}\left(\nu_{i}^{\kappa_{i}}\right)^{\dagger}\right]$, where the nilpotent operators $\nu_{i}^{\kappa_{i}}$ correspond to the Cartan-Weyl decomposition of the full $\operatorname{su}\left(d_{i}\right)$ algebra of the $i$ th element, and $x_{i}^{\kappa_{i}} \in \mathrm{C}$. We finally project the result onto the vacuum state and obtain

$$
F\left(\left\{x_{i}^{\kappa_{i}}\right\}\right)=\left\langle 0\left|\exp \left(\sum x_{i}^{\kappa_{i}}\left(\nu_{i}^{\kappa_{i}}\right)^{\dagger}\right)\right| \Psi\right\rangle,
$$

which is the generating function for the set of variables $\left\{x_{i}^{\kappa_{i}}\right\}$. This coincides with the function $F\left(\left\{\nu_{i}^{\kappa_{i}}\right\}\right)$ given by the nilpotent polynomial $F$ where all the operators $\sigma^{+}$are replaced by the corresponding variables $x$.

As long as the set $\nu_{i}^{\kappa_{i}}$ corresponds to the full algebra, introducing the generating function adds nothing new to the characterization of entanglement. The situation changes when we consider subalgebras of rank $m_{i}<r_{i}$. The generating function takes then the form

$$
F\left(\left\{x_{i}^{\kappa_{i}}\right\}\right)=\left\langle 0\left|\exp \left(\sum x_{i}^{\kappa_{i}}\left(\mu_{i}^{\kappa_{i}}\right)^{\dagger}\right)\right| \Psi\right\rangle,
$$

where the commuting nilpotent operators $\left\{\mu_{i}^{\kappa_{i}}\right\}$ belong to an algebra of rank $m_{i}$. The elements $\mu_{i}^{\kappa_{i}}$ are linear combinations $\left\{\mu_{i}^{\kappa_{i}}\right\} \subset \operatorname{span}\left\{\nu_{i}^{\kappa_{i}}\right\}$ of the nilpotent elements in the CartanWeyl decomposition $L^{z} \oplus L^{+} \oplus L^{-}$. Among the Cartan generators $\left\{\lambda^{\kappa}\right\}$, we also have to single out a subset $\left\{v^{\kappa}\right\} \subset L^{z}$ of the operators corresponding to the choice of $\left\{\mu_{i}^{\kappa_{i}}\right\}$. Note that linear combinations $\mu_{i}^{\kappa_{i}}$ of the nilpotent operators $\nu_{i}^{\kappa_{i}}$ are also nilpotent, however of higher order, that is, such that $\left(\mu_{i}^{\kappa_{i}}\right)^{p_{i}}=0$ for some integer $p_{i}^{\kappa_{i}}>1$. For example, for $\mu=t^{+}$ $+s^{+}$of Eqs. (77)-(79), one finds $\mu^{2}=u^{+} \neq 0, \mu^{3}=0$. Therefore, unlike all cases discussed so far, the generating function 
$F\left(\left\{x_{i}^{\kappa_{i}}\right\}\right)$ may contain some higher powers of the variables $x_{i}^{\kappa_{i}}$, along with the terms linear in $x_{i}^{\kappa_{i}}$.

The generating function $F\left(\left\{x_{i}^{\kappa_{i}}\right\}\right)$ may also be reduced to canonic form. This must be accomplished, however, only by resorting to local unitary operations which belong to the subalgebra of restricted local transformations,

$$
\begin{aligned}
F_{c}\left(\left\{x_{i}^{\kappa_{i}}\right\}\right)= & \langle 0| \exp \left(\sum x_{i}^{\kappa_{i}}\left(\mu_{i}^{\kappa_{i}}\right)^{\dagger}\right) \exp \left(i \sum Z_{i}^{\kappa_{i}} v_{i}^{\kappa_{i}}+R_{i}^{\kappa_{i}}\left(\mu_{i}^{\kappa_{i}}\right)^{\dagger}\right. \\
& \left.+\left(R_{i}^{\kappa_{i}}\right)^{*} \mu_{i}^{\kappa_{i}}\right)|\Psi\rangle .
\end{aligned}
$$

One may generalize the above approach to the case of restricted SL transformations, where in place of $Z_{i}^{\kappa_{i}}, R_{i}^{\kappa_{i}}$, and $\left(R_{i}^{\kappa_{i}}\right)^{*}$, one must substitute in Eq. (95) independent complex parameters. The corresponding nilpotent polynomial $F_{c}\left(\left\{\mu_{i}^{\kappa_{i}}\right\}\right)$ may then contain powers of the nilpotent variables, which is a signature of a very important fact: Entanglement is no longer necessarily associated with different subsystems, but may occur within each single element as well.

\section{Examples: Generalized entanglement for one and two spin-1 systems}

We first consider generalized entanglement in the simplest example of a three-level system (see Refs. [19,58]) and show how this result may be interpreted in terms of nilpotent variables. Though a three-level system corresponds to a full su(3) algebra, we consider it here as a spin-1 system that is, concentrate on a situation where the physical observables are restricted to the subalgebra su(2) of spin operators, $S_{ \pm}$ $=S_{x} \pm i S_{y}$ and $S_{z}$. Note that the latter are equivalent to the $u^{ \pm}+t^{\mp}$, and $\lambda^{3}$ generators of su(3), respectively. The spin states are characterized by the eigenvalues of $v=\lambda^{3}$, which is the only Cartan generator of the su(2) subalgebra. The spindown, lowest-weight state $|-1\rangle$ is chosen as the reference state. The operators $s^{+}$and $t^{-}$form the commuting nilpotent subalgebra $\{\nu\}$ of su(3) and give two other "excited" states $|0\rangle=t^{-}|-1\rangle$ and $|1\rangle=s^{+}|-1\rangle$. They characterize the quantum states of the three-level system according to the relation $|\Psi\rangle=\left(1+\alpha_{s} s^{+}+\alpha_{t} t^{-}\right)|-1\rangle$. The operator $S_{+}=u^{+}+t^{-}$is the only element of the nilpotent $\left\{\mu_{i}^{\kappa_{i}}\right\}$-subalgebra su(2) $\subset \mathrm{su}(3)$.

Now we show that the state $|0\rangle$ is generalized entangled with respect to $\mathrm{SU}(2)$. Indeed, by the unitary matrix $\mathrm{e}^{-\mathrm{i} \pi S_{x} / \sqrt{2}}$ the state $|0\rangle$ may be transformed to the state with the maximum vacuum population $\left|\psi_{C}\right\rangle=(|-1\rangle+|1\rangle) / \sqrt{2}$, which is evidently different from the reference state $|-1\rangle$. The corresponding canonic state normalized to unit reference state amplitude reads

$$
\left|\Psi_{c}\right\rangle=\left(1+s^{+}\right)|-1\rangle=\exp s^{+}|-1\rangle,
$$

hence $f_{c}=s^{+}$and $F_{c}=1+s^{+}$. We now construct the generating function of Eq. (93) by employing $S_{+}=u^{+}+t^{-}$as the only element of the nilpotent $\left\{\mu_{i}^{\kappa_{i}}\right\}$-subalgebra of su(2) for our system. This yields

$$
\begin{aligned}
F(x) & =\left\langle-1\left|\exp \left[x\left(u^{+}+t^{-}\right)^{\dagger}\right] \exp s^{+}\right|-1\right\rangle \\
& =\left\langle-1\left|\exp \left[x\left(u^{-}+t^{+}\right)\right] \exp s^{+}\right|-1\right\rangle=1+x^{2} / 2 .
\end{aligned}
$$

The presence of the quadratic term $x^{2} / 2$ is the signature of generalized entanglement.
One way to understand the meaning of this "selfentanglement" in the state $|0\rangle$ is to see it as a consequence of the fact that the operators $S_{ \pm, z}$ of su(2) cannot lift the degeneracy of the two eigenstates of the operator $\lambda_{8}$, which together with $\lambda_{3}=S_{z}$ labels the states in the unrestricted su(3) algebra. In other words, within the group of restricted local transformations, the transition from the state $|0\rangle$ can only access the state $(|-1\rangle+|1\rangle) / \sqrt{2}$ but not $(|-1\rangle-|1\rangle) / \sqrt{2}$, hence the amplitudes of the reference state $|-1\rangle$ and that of the state $|1\rangle$ are fully correlated. Alternatively, one can say that no SU(2) transformation is able to connect the state $|-1\rangle$, which is a SU(2)-GCS and is unentangled, to the state $|0\rangle$ which is a SU(3)-GCS but not a SU(2)-GCS.

For a generic $\mathrm{SU}(3)$ state $\left(1+\alpha_{s} s^{+}+\alpha_{t} t^{-}\right)|-1\rangle$, the nilpotent $\mathrm{SU}(2)$ polynomial

$$
F\left(s^{+}\right)=\left.F(x)\right|_{x \rightarrow s^{+}}
$$

is given by the generating function

$$
\begin{aligned}
F(x) & =\left\langle-1\left|\exp \left[x\left(u^{+}+t^{-}\right)^{\dagger}\right] \exp \left(\alpha_{s} s^{+}+\alpha_{t} t^{-}\right)\right|-1\right\rangle \\
& =1+\alpha_{t} x+\frac{\alpha_{s} x^{2}}{2} .
\end{aligned}
$$

The corresponding canonic nilpotent polynomial of Eq. (95) and the tanglemeter take the form

$$
F_{c}\left(S_{+}\right)=1+\alpha_{s}^{\prime} S_{+}^{2} / 2, \quad f_{c}\left(S_{+}\right)=\alpha_{s}^{\prime} S_{+}^{2} / 2,
$$

respectively, where the phase of $\alpha_{s}^{\prime}$ can be set to 0 . Therefore, generalized entanglement of a single spin-1 is characterized by a single real parameter.

Consider now a second example, that is, entanglement between two spin-1 $[19,58]$, which we describe as two threelevel systems subject to the action of $\mathrm{SU}(2) \oplus \mathrm{SU}(2)$ local operations. By analogy to the single spin-1 case, we chose nilpotent variables $\left\{\mu_{i}^{\kappa_{i}}\right\}=\left\{S_{1,2}\right\}$ in $\operatorname{su}(2) \oplus \operatorname{su}(2)$, where $S_{1,2}$ $\equiv\left(S_{+}\right)_{1,2}=u_{1,2}^{+}+t_{1,2}^{-}$, and $\left\{v^{\kappa}\right\}=\left\{\left(S_{z}\right)_{1,2}\right\}$. The state $|-1,-1\rangle$ is chosen as the SU(2) reference state. As before, the operators $s_{1}^{+}, t_{1}^{-}, s_{1,2}^{+}$, and $t_{1,2}^{-}$are the nilpotent variables in the full $\operatorname{su}(3) \oplus \operatorname{su}(3)$ algebra. A generic quantum state for a twoqutrit assembly

$$
\begin{aligned}
|\Psi\rangle= & \left(1+\alpha_{s ; 1} s_{1}^{+}+\alpha_{t ; 1} t_{1}^{-}+\alpha_{s ; 2} s_{2}^{+}+\alpha_{t ; 2} t_{2}^{-}+\alpha_{s, s} s_{2}^{+} s_{1}^{+}+\alpha_{t, s} s_{2}^{+} t_{1}^{-}\right. \\
& \left.+\alpha_{t, s} t_{2}^{-} s_{1}^{+}+\alpha_{t, t} t_{2}^{-} t_{1}^{-}\right)|-1,-1\rangle,
\end{aligned}
$$

is characterized by the nilpotent polynomials

$$
\begin{aligned}
F\left(\left\{s^{+}, t^{-}\right\}\right)= & 1+\alpha_{s ; 1} s_{1}^{+}+\alpha_{t ; 1} t_{1}^{-}+\alpha_{s ; 2} s_{2}^{+}+\alpha_{t ; 2} t_{2}^{-}+\alpha_{s, s} s_{2}^{+} s_{1}^{+} \\
& +\alpha_{t, s} s_{2}^{+} t_{1}^{-}+\alpha_{t, s} t_{2}^{-} s_{1}^{+}+\alpha_{t, t} t_{2}^{-} t_{1}^{-}, \\
f\left(\left\{s^{+}, t^{-}\right\}\right)= & \alpha_{s ; 1} s_{1}^{+}+\alpha_{t ; 1} t_{1}^{-}+\alpha_{s ; 2} s_{2}^{+}+\alpha_{t ; 2} t_{2}^{-}+\left(\alpha_{s, s}\right. \\
& \left.-\alpha_{s ; 2} \alpha_{s ; 1}\right) s_{2}^{+} s_{1}^{+}+\left(\alpha_{t, s}-\alpha_{s ; 2} \alpha_{t ; 1}\right) s_{2}^{+} t_{1}^{-}+\left(\alpha_{s, t}\right. \\
& \left.-\alpha_{t ; 2} \alpha_{s ; 1}\right) t_{2}^{-} s_{1}^{+}+\left(\alpha_{t, t}-\alpha_{t ; 2} \alpha_{t ; 1}\right) t_{2}^{-} t_{1}^{-}
\end{aligned}
$$

whereas for the SU(2) characterization, Eq. (94) gives the generating function 


$$
\begin{aligned}
F(x, y)= & \langle-1,-1| \exp \left[x\left(u_{1}^{+}+t_{1}^{-}\right)^{\dagger}+y\left(u_{2}^{+}+t_{2}^{-}\right)^{\dagger}\right] \\
& \times F\left(\left\{s^{+}, t^{-}\right\}\right)|-1,-1\rangle .
\end{aligned}
$$

Direct calculation yields

$$
\begin{aligned}
F(x, y)= & 1+\alpha_{s ; 1} \frac{x^{2}}{2}+\alpha_{t ; 1} x+\alpha_{s ; 2} \frac{y^{2}}{2}+\alpha_{t ; 2} y+\alpha_{t, t} x y+\alpha_{t, s} x \frac{y^{2}}{2} \\
& +\alpha_{s t} \frac{x^{2}}{2} y+\alpha_{s, s} \frac{x^{2}}{2} \frac{y^{2}}{2} .
\end{aligned}
$$

This finally results in the nilpotent polynomial

$$
\begin{aligned}
F\left(S_{1}, S_{2}\right)= & 1+\alpha_{s ; 1} \frac{S_{1}^{2}}{2}+\alpha_{t ; 1} S_{1}+\alpha_{s ; 2} \frac{S_{2}^{2}}{2}+\alpha_{t ; 2} S_{2}+\alpha_{s, s} \frac{S_{2}^{2}}{2} \frac{S_{1}^{2}}{2} \\
& +\alpha_{t, s} \frac{S_{2}^{2}}{2} S_{1}+\alpha_{s t} S_{2} \frac{S_{1}^{2}}{2}+\alpha_{t, t} S_{2} S_{1},
\end{aligned}
$$

where the subscripts + of the nilpotent variables $S$ are implicit. In the canonic form maximizing the population of the reference state, the population of the states $|0,-1\rangle$ and $|-1,0\rangle$ vanish, whereby one obtains

$$
\begin{aligned}
F_{c}\left(S_{1}, S_{2}\right)= & 1+\alpha_{s ; 1} \frac{S_{1}^{2}}{2}+\alpha_{s ; 2} \frac{S_{2}^{2}}{2}+\alpha_{s, s} \frac{S_{2}^{2}}{2} \frac{S_{1}^{2}}{2}+\alpha_{t, s} \frac{S_{2}^{2}}{2} S_{1} \\
& +\alpha_{s t} S_{2} \frac{S_{1}^{2}}{2}+\alpha_{t, t} S_{1} S_{2}, \\
f_{c}\left(S_{1}, S_{2}\right)= & \beta_{s ; 1} \frac{S_{1}^{2}}{2}+\beta_{t, t} S_{1} S_{2}+\beta_{s t} \frac{S_{1}^{2}}{2} S_{2}+\beta_{s ; 2} \frac{S_{2}^{2}}{2}+\beta_{t, s} S_{1} \frac{S_{2}^{2}}{2} \\
& +\beta_{s, s} \frac{S_{2}^{2}}{2} \frac{S_{1}^{2}}{2},
\end{aligned}
$$

where $\beta_{s, s}=\alpha_{s, s}-2 \alpha_{t, t}{ }^{2}$, and all other $\beta=\alpha$. As before, by exploiting the freedom of phase transformations on the nilpotent variables, the parameters $\alpha_{s ; 1}$ and $\alpha_{s ; 2}$ (or $\beta_{s ; 1}$ and $\beta_{s ; 2}$ ) characterizing generalized entanglement within each of the three-level systems can be set real, and we are left with four complex numbers $\alpha_{s, s}, \alpha_{t, s}, \alpha_{s t}$, and $\alpha_{t, t}$ characterizing the generalized interspin entanglement.

The following question may also be relevant: How can we characterize generalized entanglement under SL transformations? For qubits, the resulting classification is based on the dynamic equation (53), and the conditions Eqs. (64) and (65) of the exponential decrease of the chosen coefficients in the course of controlled local $\operatorname{SL}(2, \mathrm{C})$ dynamics. In order to suggest a strategy for characterizing an ensemble of spin-1 elements, we note that an analog of Eq. (53) may be derived for nilpotent polynomials on $S_{i}$ with the help of the analogs of Eqs. (40). A requirement of eliminating certain terms in $f$ by a proper choice of the local $\operatorname{SL}(2, \mathrm{C})$ control parameters may also be imposed by analogy. In particular, the part of $f$ containing only multilinear terms in $S_{i}$ may be reduced to the SL(2) canonic form which, up to the replacement $\sigma_{i}^{+} \mapsto S_{i}$, is identical to that of qubits. This determines the canonic form of the entire $f$, whereas the terms containing at least one $S_{i}^{2}$ factor comprise a generic polynomial with the coefficient specified in the process of reducing the multilinear part to the canonic form.

\section{Transformation of the nilpotential under change of partition}

Last, consider a situation which, in a sense, is dual to the above-discussed scenario of generalized entanglement, whereby the ranks of the algebras employed for the entanglement classification exceed the dimensions of the local Hilbert space of the elements. This is the case of a partition of a composite system, where each part may itself contain multiple elements. Thus, from an initial assembly we compose a new one by considering groups of elements as new elements and by describing the quantum state of each group by a single, collective quantum number. One can say that the new assembly results from merging of elements in the old one.

We begin with the example of the $n$-qubit system characterized by the nilpotential of Eq. (15),

$$
f\left(\left\{\sigma_{i}^{+}\right\}\right)=\sum_{\left\{k_{i}\right\}=0,1} \beta_{k_{n}, k_{n-1} \cdots k_{1}} \prod_{i=1}^{n}\left(\sigma_{i}^{+}\right)^{k_{i}}
$$

partitioned in three parts $A, B$, and $C$, each of which contains $n_{A}, n_{B}$, and $n_{C}$ qubits, respectively. The particular case $C$ $=\varnothing$ recovers the bipartite setting. The new assembly thus consists of three elements with $d_{A}=2^{n_{A}}, d_{B}=2^{n_{B}}$, and $d_{C}$ $=2^{n C}$.

By exploiting a standard Hubbard-Stratonovich procedure [79], we may represent the polynomial $F=e^{f}$ corresponding to Eq. (108) in the form of an integral

$$
F\left(\left\{\sigma_{i}^{+}\right\}\right)=\int e^{f\left(\left\{z_{i}\right\}\right)} \prod_{i=1}^{n} e^{-\left|z_{i}\right|^{2}+z_{i}^{*} \sigma_{i}^{+}} \frac{d^{n} z_{i} d^{n} z_{i}^{*}}{\pi},
$$

where the integration must be performed independently over both the complex variables $z_{i}$ and their complex conjugates $z_{i}^{*}$. This suggests a straightforward separation of the system into three parts,

$$
\begin{aligned}
F|0\rangle= & \int \exp \left(\sum_{i=n_{A}+n_{B}+1}^{n} z_{i}^{*} \sigma_{i}^{+}\right)|0\rangle_{C} \exp \left(\sum_{i=n_{A}+1}^{n_{A}+n_{B}} z_{i}^{*} \sigma_{i}^{+}\right) \\
& \times|0\rangle_{B} \exp \left(\sum_{i=0}^{n_{A}} z_{i}^{*} \sigma_{i}^{+}\right)|0\rangle_{A} e^{f\left(\left\{z_{i}\right\}\right)} \prod_{i=1}^{n} e^{-\left|z_{i}\right|^{2}} \frac{d^{n} z_{i} d^{n} z_{i}^{*}}{\pi},
\end{aligned}
$$

where $|0\rangle_{A ; B ; C}$ denote vacuum states of the new elements, which we still can choose as product states of qubits included within the new elements.

Let the Cartan subalgebras and the commuting nilpotent elements of the $\operatorname{su}\left(2^{n_{A}}\right), \operatorname{su}\left(2^{n_{B}}\right)$, and $\operatorname{su}\left(2^{n_{C}}\right)$ algebras be denoted by $\left\{\lambda_{A}^{\kappa_{A}}\right\}\left\{\nu_{A}^{\kappa_{A}}\right\},\left\{\lambda_{B}^{\kappa_{B}}\right\}\left\{\nu_{B}^{\kappa_{B}}\right\}$, and $\left\{\lambda_{C}^{\kappa_{C}}\right\}\left\{\nu_{C}^{\kappa_{C}}\right\}$, respectively, and let the state of the assembly be characterized by a nilpotent polynomial on $\nu^{\kappa}$ upon noticing that $\left(\nu^{\kappa}\right)^{2}=0$. This yields 


$$
\begin{aligned}
F\left(\left\{\nu_{A}^{\kappa_{A}}, \nu_{B}^{\kappa_{B}}, \nu_{C}^{\kappa_{C}}\right\}\right)= & \int\left(1+\sum_{\kappa_{A}}\left\langle\left. 0\right|_{A}\left(\nu_{A}^{\kappa_{A}}\right)^{\dagger} e^{\left.\sum_{i=1}^{n_{A}} z_{i}^{*} \sigma_{i}^{+}|0\rangle_{A} \nu_{A}^{\kappa_{A}}\right)}\right.\right. \\
& \times\left(1+\sum_{\kappa_{B}}\left\langle\left. 0\right|_{B}\left(\nu_{B}^{\kappa_{B}}\right)^{\dagger} e^{\left.\sum_{i=n_{A}+n^{+}+n_{B}}^{*} z_{i}^{*} \sigma_{i}^{+}|0\rangle_{B} \nu_{B}^{\kappa_{B}}\right)}\right.\right. \\
& \times\left(1+\sum_{\kappa C}\left\langle\left. 0\right|_{C}\left(\nu_{C}^{\kappa_{C}}\right)^{\dagger} e^{\left.\sum_{i=n_{A}+n_{B}+1}^{n} z_{i}^{*} \sigma_{i}^{+}|0\rangle_{C} \nu_{C}^{\kappa_{C}}\right)}\right.\right. \\
& \times e^{f\left(\left\{z_{i}\right\}\right)} \prod_{i=1}^{n} e^{-\left|z_{i}\right|^{2}} \frac{d^{n} z_{i} d^{n} z_{i}^{*}}{\pi} .
\end{aligned}
$$

One may rewrite this in the form

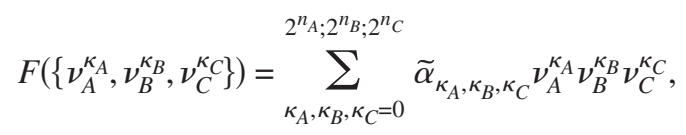

where the expression

$$
\begin{aligned}
\tilde{\alpha}_{\kappa_{A}, \kappa_{B}, \kappa_{C}}= & \int\left\langle\left. 0\right|_{A}\left(\nu_{A}^{\kappa_{A}}\right)^{\dagger} \exp \left(\sum_{i=0}^{n_{A}} z_{i}^{*} \sigma_{i}^{+}\right)\right. \\
& \times|0\rangle_{A}\left\langle\left. 0\right|_{B}\left(\nu_{B}^{\kappa_{B}}\right)^{\dagger} \exp \left(\sum_{i=n_{A}+1}^{n_{A}+n_{B}} z_{i}^{*} \sigma_{i}^{+}\right)\right. \\
& \times|0\rangle_{B}\left\langle\left. 0\right|_{C}\left(\nu_{C}^{\kappa_{C}}\right)^{\dagger} \exp \left(\sum_{i=n_{A}+n_{B}+1}^{n} z_{i}^{*} \sigma_{i}^{+}\right)\right. \\
& \times|0\rangle_{C} e^{f\left(\left\{z_{i}\right\}\right)} \prod_{i=1}^{n} e^{-\left|z_{i}\right|^{2}} \frac{d^{n} z_{i} d^{n} z_{i}^{*}}{\pi},
\end{aligned}
$$

explicitly gives the coefficients of the new nilpotent polynomials characterizing entanglement in the new assembly. Here, the identity operators $\nu_{A}^{0}, \nu_{B}^{0}$, and $\nu_{C}^{0}$ are included in the sets $\left\{\nu_{A}^{\kappa_{A}}\right\},\left\{\nu_{B}^{\kappa_{B}}\right\}$, and $\left\{\nu_{C}^{\kappa_{C}}\right\}$, respectively. Note that for bipartite and tripartite entanglement, the expressions for $\tilde{\alpha}$ and for $\widetilde{\beta}$ are identical, provided that one eliminates terms of $F$ linear in $\nu$ by local transformations $\mathrm{SU}\left(2^{n_{A}}\right) \otimes \mathrm{SU}\left(2^{n_{B}}\right)$ $\otimes \mathrm{SU}\left(2^{n_{C}}\right)$, and normalizes the reference state population to unity. Also note that for a bipartite case, the sum $\rho_{\kappa_{A}, \kappa_{A}^{\prime}}$ $=\sum_{\kappa_{B}} \widetilde{\alpha}_{\kappa_{A}, \kappa_{B}} \widetilde{\alpha}_{\kappa_{A}^{\prime}, \kappa_{B}}^{*}$ gives the density matrix of part $A$ normalized to unit population of the reference state.

The generalization of expressions Eq. (112) and (113) to a larger number of new elements is straightforward,

$$
\begin{aligned}
& F\left(\left\{\nu_{A}^{\kappa_{A}}, \ldots, \nu_{W}^{\kappa_{W}}\right\}\right)=\sum_{\kappa_{A}, \ldots, \kappa_{W}=0}^{2^{n_{A} ; \cdots ; 2^{n_{W}}}} \tilde{\alpha}_{\kappa_{A}, \ldots, \kappa_{W}} \nu_{A}^{\kappa_{A}} \cdots \nu_{W}^{\kappa_{W}}, \\
& f\left(\left\{\nu_{A}^{\kappa_{A}}, \ldots, \nu_{W}^{\kappa_{W}}\right\}\right)=\sum_{\kappa_{A}, \ldots, \kappa_{W}=0}^{2^{n_{A}, \cdots, 2^{n_{W}}}} \tilde{\beta}_{\kappa_{A}, \ldots, \kappa_{W}} \nu_{A}^{\kappa_{A}} \cdots \nu_{W}^{\kappa_{W}},
\end{aligned}
$$

where the integrand of Eq. (113) for $\widetilde{\alpha}_{\kappa_{A}, \ldots, \kappa_{W}}$ now contains more factors

$$
\left\langle\left. 0\right|_{K}\left(\nu_{K}^{\kappa_{A}}\right)^{\dagger} \exp \left(\sum z_{i}^{*} \sigma_{i}^{+}\right) \mid 0\right\rangle_{K}
$$

with $i$ running over all qubits included in the new element set $K$. Once the reference state amplitude is normalized to 1 , the nilpotential $f$ may be found by direct calculation of $\ln F$. This yields relations among $\widetilde{\alpha}$ and $\widetilde{\beta}$ that even for the canonic state do not coincide when the assembly comprises more than three new elements.

Finally, one may raise the following question: What could be a reasonable choice of a state marking an orbit, in the case where the dimensions of elements become large, and the exponential complexity makes the identification of a global maximum of the reference state population an intractable problem? One possibility is to rely on dynamics and manipulations exclusively with the nilpotential $f$, aiming at eliminating the coefficients $\beta$ of all the states connected to the states $|k, \cdots, k\rangle$ by a single local transformation, as illustrated in Fig. 4. However, we note that such a choice, though providing a unique characterization of entanglement, may yield canonic forms not corresponding to the maximum population of the reference state. Hence it may be ambiguous, leading to different polynomials for the same orbit, as it is the case of the example presented in Eq. (25) of Ref. [39]. Still, such a choice might have some advantages in view of "operational compatibility" important for large ensembles, since it relies exclusively on the manipulations with the extensive polynomial $f$ and does not invoke $F$, thereby avoiding the need for the exponentially long procedure of the $F \Leftrightarrow f$ conversion. Another advantage is that the generalization to the SL case is straightforward.

\section{SUMMARY AND OUTLOOK}

We conclude by summarizing the general idea of our entanglement description in composite quantum systems, based on the selection of a product reference state and on the introduction of appropriate local nilpotent operators. We also summarized the results achieved so far and mention possible future applications of the nilpotent formalism to other physical problems relevant to entanglement theory and quantum information science.

Dealing with a physical system composed of distinguishable parts, we need to specify among which subsets of the parts we wish to consider. In order to avoid confusion, we consider each of these subsets as a single element and characterize it by a single quantum number. We call assembly the collection of all the elements.

The main motivation for introducing the nilpotent variables technique is to obtain a characterization of entanglement by extensive quantities, i.e., sums of the characteristics of the unentangled parts of the system. This approach critically relies on an important property of nilpotent polynomials: any analytical function of a polynomial is also a polynomial. Thus, a key role is played by the logarithm function, which enables one to relate the product state to a sum of independent terms, each representing a part of the system unentangled with the rest. The extensive characteristic, nilpotential $f$, that emerges, for a product quantum state is indeed a sum of nilpotentials of the unentangled parts, 
whereas the presence of entanglement among the elements of different parts is represented by the corresponding cross terms. Verifying whether or not such terms are present in the nilpotential thus serves as the entanglement criterion.

By the very meaning of entanglement among elements of an assembly, these characteristics should be insensitive to local transformations, which result from arbitrary reversible (both unitary and not unitary) individual manipulations of the elements. Therefore, the entire orbit of states, that is the manifold of all states of the assembly that can be reached from a given initial state by local operations, should correspond to the same parameter values characterizing entanglement. The number of these parameters, that is the dimension $D$ of the orbit coset, depends on the type of local transformations allowed. For both unitary SU and nonunitary SL transformations, we propose a canonic form of the assembly state, which depends on $D$ parameters and serves as the orbit marker. For qubits, the canonic form relies on the choice of a reference product state of the assembly, the vacuum, and on maximization of the population of this state via local transformations.

Given the canonic state of an assembly of qubits, we represent it in the form of a polynomial $F_{c, C}\left(\left\{\sigma_{i}^{+}\right\}\right)$of the raising operators $\sigma_{i}^{+}$acting on the vacuum state. After normalizing to unit vacuum state amplitude, we calculate another polynomial, the tanglemeter $f_{c, C}\left(\left\{\sigma_{i}^{+}\right\}\right)=\ln F_{c, C}\left(\left\{\sigma_{i}^{+}\right\}\right)$, which depends on exactly $D$ parameters and contains all the information about entanglement in the form of the coefficients standing in front of different nilpotent monoms $\Pi_{i} \sigma_{i}^{+}$. Subscripts $C$ and $c$ refer to the SU or SL group of local transformations, respectively. In contrast to the state given by the polynomial $F_{c, C}$, the tanglemeter $f_{c, C}$ is an extensive quantity. In statistical physics, extensive quantities scale linearly with the size of the ensemble, like free energy given by the logarithm of the partition function. The tanglemeter has similar additive properties: for a composite system representing a set of unentangled parts, the tanglemeter equals the sum of the tanglemeters of the constituent parts. Straightforward inspection of the second derivatives $\partial^{2} f_{c, C} / \partial \sigma_{i \in A}^{+} \partial \sigma_{j \in B}^{+}$allows one to check whether or not groups $A$ and $B$ of elements are entangled.

We have presented several examples of tanglemeters for systems of a few qubits. Still, there may exist a certain state, for which the tanglemeter, though dependent on the same number $D$ of parameters, cannot have the chosen structure. These states comprise singular classes. In the four-qubit example, we have shown in detail how a classification may be constructed by considering local infinitesimal transformations sequentially diminishing the coefficients of the monoms to be eliminated. This procedure is described by a set of differential equations for the coefficients of $f$ and can be seen as a control process, when we impose appropriate feedback conditions on the parameters of continuous local transformations. By properly adjusting these parameters to current values of the coefficients of the nilpotential, we rapidly reach the tanglemeter $f_{c}$ in the limit $t \rightarrow \infty$. The latter thus appears as a stationary stable point of the set of equations with feedback. However, the proper choice of the parameters required for elimination of the monoms cannot be made in certain domains in the space of the nilpotential coefficients, where this procedure fails due to "loss of complete controllability." In these domains, the determinants of matrices relating the time derivatives of the coefficient with the parameters of the local transformations vanish. The cases where one, two, three, or all four eigenvalues of the determinant equal zero correspond to different entanglement classes. A more systematic exploration of this classification in larger assemblies of qubits appears to be an interesting task for immediate future research.

We have demonstrated how the nilpotent polynomial technique, initially developed for qubits, may be extended to $d$-level elements, qudits, each of which has different dimension $d_{i}$. In this case, a larger number of the nilpotent variables per subsystem is required for the construction of the polynomials. The salient features of the proposed procedure may be summarized as follows.

(i) We start by choosing a reference state $|0\rangle_{i}$ for an individual element and a corresponding state for the composite quantum system, an assembly consisting of $d$-level systems, $|\mathrm{O}\rangle=|0, \ldots, 0\rangle$. All other states are obtained by the action on $|\mathrm{O}\rangle$ by polynomials of nilpotent local operators. For qubits, the latter are the spin raising operators $\sigma_{i}^{+}$. For qudits, we invoke $d-1$ nilpotent commuting operators $\nu_{i}^{\kappa_{i}}$ that create $d_{i}-1$ excited states $\left|\kappa_{i}\right\rangle=\nu_{i}^{\kappa_{i}}|0\rangle_{i}$ out of the vacuum state $|0\rangle_{i}$. This choice of basis is natural in the framework of the Cartan-Weyl decomposition of the $\operatorname{su}(d)$ algebra. Thereby any state of the composite system may be represented by a polynomial $F\left(\nu_{i}^{\kappa_{i}}\right)$ acting on the reference state.

(ii) By applying local transformations to a given assembly state we bring it into a certain canonic form. For systems of qubits, the latter is characterized by maximum population of the reference state $|\mathrm{O}\rangle$. In the case of qudits, we need to additionally maximize the population of the maximum symmetric excited states. When the dimensions $d_{i}$ of all elements are equal, these are the states $|1, \ldots, 1\rangle, \ldots,|\kappa, \ldots, \kappa\rangle \ldots, \mid d$ $-1, \ldots, d-1\rangle$, while for different $d_{i}$, in this sequence, we replace $\kappa$ by $d_{i}-1$ for the elements of the dimension $d_{i}<\kappa$.

(iii) Any analytical function of a polynomial of nilpotent arguments $\nu_{i}^{\kappa_{i}}$ is also a polynomial. The logarithm of the canonic polynomial $F_{c}$, the tanglemeter, $f_{c}$ has coefficients that by construction are invariant with respect to local transformations. This offers a simple way to systematically list all the invariant characteristics of entanglement for a system of an arbitrary number of qudits.

Another extension of the technique applies to a situation relevant to the generalized entanglement setting, whereby the algebra of local transformations has a rank strictly less than that suggested by the dimension of the elements. In such a case, the number of nilpotent variables per element is less than $d-1$, and moreover, the form of the nilpotent polynomial is sensitive to the choice of reference state, thus being "reference" and "observable" dependent. Identification of classes for large elements and for generalized entanglement based on appropriate dynamic equations for the nilpotential are two immediate open questions deserving further investigation.

Manipulation of quantum assemblies and, in particular, quantum computation, implies application of both local and nonlocal two-particle gate transformations. This is also the case for a system composed of naturally interacting ele- 
ments, e.g., spin chains, cold Rydberg gases, and arrays of cold two-level atoms trapped in standing electromagnetic wave. The Schrödinger equation yields a dynamic equation for the nilpotent polynomials - a linear one for the polynomial $F$, which is in turn linear in the state amplitudes, and a nonlinear one for $f=\ln F$. For an ensemble of $n$ qubits, both dynamic equations involve $\sim 2^{n}$ variables, the polynomial coefficients and their time derivatives. At a first glance, linear equations are always simpler. However, this is not necessarily the case: we have shown an example of universal evolution that yields an equation for $f$, which is equivalent to the classical Hamilton-Jacobi equation in just $n$ dimensions. How far does this analogy go? What are the consequences for entanglement dynamics and, in particular, for quantum algorithms? These are yet additional intriguing questions that deserve to be further addressed.

Finally, one could conceive applications of the nilpotent polynomial technique for analytical investigations of entanglement in correlated many-body systems -including the problem of better characterizing quantum phase transitions [19] — and extend the present consideration to the case where nonunitary decoherence and dissipation effect are included in the dynamics. In an even broader context, one can think of employing this technique as a framework for establishing deeper relations between entanglement dynamics $[18,21]$ and the known exactly solvable problems of statistical mechanics and complex quantum systems $[80,81]$.

\section{ACKNOWLEDGMENTS}

Two of the authors (A.M.) is grateful to J. W. Clark for support during this work. The author (A.M.) also acknowledges the support by the U.S. National Science Foundation under Grant No. PHY-0140316 and by the Nipher Fund. One of the authors (V.M.A.) gratefully acknowledges the support of QUACS EC network grant. Two of the authors (A.M. and V.M.A.) are grateful to M. S. Byrd, J. W. Clark, I. Dumer, J. Siewert, V. Kaç, V. Kravtsov, I. Lerner, and V. Youdson for useful discussions and communication, as well as to the organizers of Madeira Math Encounters XXIX and to the ICTP institute for their hospitality. One of the authors (L.V.) gratefully acknowledges partial support from Constance and Walter Burke through their Special Project Fund in Quantum Information Science.

\section{APPENDIX A: DIMENSION OF COSETS}

(a) Two qubits: The fact that the expression Eq. (4) is invariant implies that the counting $2^{n+1}-3 n-2$ of the number of invariants breaks for $n=2$. That means that out of $3 n=6$ local transformations, only five act faithfully (nontrivially). In other words, there is a certain local transformation which leaves a generic wave function intact. One can amuse oneself and find it explicitly. A generic infinitesimal local transformation is

$$
\delta \psi_{i j}=i P_{2}^{\kappa}\left(\sigma_{2}^{\kappa}\right)_{i i,} \psi_{i, j}+i P_{1}^{\kappa}\left(\sigma_{1}^{\kappa}\right)_{j j}, \psi_{i j},
$$

Leaving only the components $\psi_{00}$ and $\psi_{11}$ (as dictated by the canonic form), and writing $P_{r}^{\kappa} \sigma_{r}^{\kappa}=P_{r}^{z} \sigma^{z}+P_{r}^{-} \sigma^{+}+P_{r}^{+} \sigma^{-}$with $r=1,2$, we arrive at the system of equations

$$
\begin{aligned}
& -i \delta \psi_{11}=\left(P_{1}^{z}+P_{2}^{z}\right) \psi_{11}=0, \\
& -i \delta \psi_{00}=-\left(P_{1}^{z}+P_{2}^{z}\right) \psi_{00}=0, \\
& -i \delta \psi_{01}=P_{1}^{-} \psi_{00}+P_{2}^{+} \psi_{11}=0 \\
& -i \delta \psi_{10}=P_{2}^{-} \psi_{00}+P_{1}^{+} \psi_{11}=0
\end{aligned}
$$

From the last two equations, $P_{1}^{+}=P_{2}^{+}=0$ (for generic $\psi_{00}$, $\psi_{11}$ ), while the first and the second ones give the same condition $P_{1}^{z}+P_{2}^{z}=0$ [or $\phi_{1}+\phi_{2}+\varphi_{1}+\varphi_{2}=0$ in the notation of Eq. (10)], i.e., we have a one-parametric set of local transformations that leave $\psi_{i j}$ intact.

(b) Three qubits: Take a special canonic state with only four nonzero components: $\psi_{011}, \psi_{101}, \psi_{110}$, and $\psi_{000}=1$. The infinitesimal local transformations are

$$
\delta \psi_{i j k}=i P_{3}^{\kappa}\left(\sigma_{3}^{\kappa}\right)_{i i^{\prime}} \psi_{i^{\prime} j k}+i P_{2}^{\kappa}\left(\sigma_{2}^{\kappa}\right)_{j j^{\prime}} \psi_{i j^{\prime} k}+i P_{1}^{\kappa}\left(\sigma_{1}^{\kappa}\right)_{k k^{\prime}} \psi_{i j k^{\prime}} .
$$

Let us show that $\delta \psi_{i j k}=0$ implies $P_{r}^{\kappa}=0$ for all $\kappa= \pm, z$, and $r=1,2,3$. The corresponding system of equations is

$$
\begin{gathered}
-i \delta \psi_{000}=-\left(P_{3}^{z}+P_{2}^{z}+P_{1}^{z}\right)=0 \\
-i \delta \psi_{100}=P_{2}^{+} \psi_{110}+P_{1}^{+} \psi_{101}+P_{3}^{-}=0 \\
-i \delta \psi_{010}=P_{3}^{+} \psi_{110}+P_{1}^{+} \psi_{011}+P_{2}^{-}=0 \\
-i \delta \psi_{001}=P_{3}^{+} \psi_{101}+P_{2}^{+} \psi_{011}+P_{1}^{-}=0 \\
-i \delta \psi_{011}=\left(P_{2}^{z}+P_{1}^{z}-P_{3}^{z}\right) \psi_{011}=0 \\
-i \delta \psi_{101}=\left(P_{1}^{z}+P_{3}^{z}-P_{2}^{z}\right) \psi_{101}=0 \\
-i \delta \psi_{110}=\left(P_{3}^{z}+P_{2}^{z}-P_{1}^{z}\right) \psi_{110}=0 \\
-i \delta \psi_{111}=P_{3}^{-} \psi_{011}+P_{2}^{-} \psi_{101}+P_{1}^{-}
\end{gathered}
$$

Equations (119)-(121) have the form of a homogeneous linear system of six equations for the real and imaginary parts of $P_{r}^{+}$. One can be convinced that the determinant of this system does not vanish for generic $\psi_{011}, \psi_{101}, \psi_{110}$ and this implies that the only solution is $P_{r}^{+}=0$. Equations (A8)-(A10) give in turn a homogeneous system for the three parameters $P_{r}^{z}=0$. Its determinant does not vanish for nonzero $\psi_{011}, \psi_{101}, \psi_{110}$ and this implies $P_{r}^{z}=0$. Q.E.D.

Note that this statement would not be correct for any pure state. The state having $\psi_{000}$ as the only nonvanishing component is annihilated by two linearly independent generators (with $P_{1}^{z}+P_{2}^{z}=0$ and $P_{1}^{z}+P_{3}^{z}=0$ ). For some other choice, there is only one trivially acting generator. But for a generic state there is none.

(c) $n>3$ qubits: The local transformations involve $3 n$ parameters $P_{j}^{\kappa}$. Introduce, as before, $P_{j}^{ \pm}=P_{j}^{x} \pm i P_{j}^{y}$. Take the state with $\psi_{000}=1$ and $n$ other nonzero components $\psi_{01 \cdots 1}$, $\psi_{101 \cdots 1}, \ldots, \psi_{1 \cdots 10}$. Consider $\delta \psi_{01 \cdots 1}$, etc. We obtain a system of $n$ linear homogeneous equations for $n$ real variables $P_{i}^{z}$. The matrix of the system 


$$
M=\left(\begin{array}{cccc}
-1 & 1 & \cdots & 1 \\
1 & -1 & \cdots & 1 \\
\cdots & \cdots & \cdots & \cdots \\
1 & \cdots & \cdots & -1
\end{array}\right)
$$

[cf. Eqs. (A8)-(A10)] has a nonvanishing determinant for $n$ $>2$. Indeed, $M=A-2 I$, where $A$ is the matrix with all components equal to 1 and $I$ is the unit matrix. But $A$ is a matrix of rank 1. It has $n-1$ degenerate eigenvalues $\lambda=0$ and one eigenvalue $\lambda=n$. And $\lambda=2$ is not an eigenvalue. It follows from this that the matrix $M$ does not have zero eigenvalues and the only solution to the equation system is $P_{j}^{z}=0$.

Consider now $\delta \psi_{\left\{k_{n}\right\}}$, where the set $\left\{k_{n}\right\}$ involves two zeros and $n-2$ unities. We obtain a system of $n(n-1) / 2$ linear homogeneous equations for $n$ complex variables $P_{j}^{+}$. Let us prove by induction that it has only zero solutions for generic $\psi_{01 \cdots 1}$, etc. Consider first $\delta \psi_{\left\{k_{n}\right\}}$, with the unity at the leftmost position $\left(\left\{k_{n}\right\}=\left\{1,\left\{k_{n-1}\right\}\right\}\right)$. The equation system for $P_{2}^{+}, \ldots, P_{n}^{+}$derived from this has the same form as the equation system $\delta \psi_{\left\{k_{n-1}\right\}}$ in the case of $n-1$ qubits. By the inductive assumption, it has only zero solutions. Knowing that $P_{2, \ldots, n}^{-}=0$ and considering, say, the equation

$$
\delta \psi_{001 \cdots 1}=P_{1}^{+} \psi_{10 \cdots 1}+P_{2}^{+} \psi_{01 \cdots 1}=0,
$$

we derive that in addition that $P_{1}^{+}=0$.

This proves that no infinitesimal transformation leaving the generic $n$-qubit state exists for $n>2$.

(d) Remark on the invariance of the canonic form: Let us consider the canonic state suggested by Eq. (9) and try to prove that this form is unique. Consider infinitesimal local transformations $1+i \Sigma_{i} P_{i}^{+} \sigma_{i}^{-}+i \Sigma_{i} P_{i}^{-} \sigma_{i}^{+}$of a canonic state vector and require that they do not bring about linear in $\sigma_{j}^{+}$ terms. The requirement means that

$$
\delta \psi_{10 \cdots 0}=\cdots=\delta \psi_{0 \cdots 01}=0
$$

and implies

$$
\begin{gathered}
P_{1}^{-} \psi_{0 \cdots 0}+P_{2}^{+} \psi_{110 \cdots 0}+\cdots+P_{n}^{+} \psi_{10 \cdots 01}=0, \\
\cdots, \\
P_{1}^{+} \psi_{10 \cdots 01}+P_{2}^{+} \psi_{010 \cdots 01}+\cdots+P_{n}^{-} \psi_{0 \cdots 0}=0 .
\end{gathered}
$$

This is a system of $n$ complex equations for $n$ complex parameters $P_{j}^{-}=\left(P_{j}^{+}\right)^{*}$, which we have encountered in Eq. (64). It can be cast in the form

$$
P_{j}^{-}+\sum_{i=1}^{n} P_{i}^{+} M_{i, j}=0 .
$$

Upon exploiting Eq. (60), the above coincides with the righthand side of Eq. (63), which we encountered discussing the dynamic reduction of nilpotentials to the canonic form. There we saw that all eigenvalues of $M_{i, j}$ are never equal to 1 for generic states in the canonic form, since this would contradict the requirement of the maximum vacuum state population. Therefore, the determinant of the system Eq. (A14) does not vanish in the generic case, hence the system
Eq. (A13) has only the trivial solution $\alpha_{j}=0$. The canonic form of the generic state is thus unique up to phase transformations, unless the phases are specified by additional requirements.

\section{APPENDIX B: GRAPH APPROACH. FOUR-QUBIT sl-CLASSIFICATION}

In this appendix we provide a physical meaning for the coefficients of the four-qubit tanglemeter, by relating them to concurrence and 3-tangle. Based on these results, in the first section of this appendix we construct graphs that illustrate the entanglement topology. In the second section, we summarize the sl-entanglement classes for four qubits as they emerge from the dynamic equations for the tanglemeter.

(a) Graphical interpretation of the tanglemeter coefficients: Starting with two qubits in a pure state, the tanglemeter involves only one coefficient, $\beta_{3}$, in terms of which we can express the von Neumann entropy $S_{v N}=-\operatorname{Tr}\left(\rho_{A} \log \rho_{A}\right)$ or the concurrence, by

$$
C_{12}=2 \sqrt{\operatorname{det} \rho}=2 \beta_{11} /\left(1+\beta_{11}^{2}\right) .
$$

Since the tanglemeter in this case is nothing but the Schmidt decomposition, nothing new is introduced; a nonzero $\beta$ coefficient implies the presence of bipartite entanglement among the two qubits.

For three qubits in a pure state, both $C$ and $\tau$ can be expressed in terms of the amplitudes $\psi$ of the su-canonical state Eqs. (29) and (30). These expressions are simpler than for a noncanonical state vector, since they do not contain the amplitudes $\psi_{100}, \psi_{010}$, and $\psi_{001}$.

For more than three qubits one can evaluate the concurrence. Since the expressions are rather complicated, we shall instead employ Peres' separability criterion of partial transposition [25]: the qubits are not entangled iff the eigenvalues of the partially transposed reduced density matrix of qubits 1 and 2 are non-negative. The first case we considered is that in which only bilinear terms are present in the canonical state of four qubits. The eigenvalues of the partial transposed reduced density matrix are found to satisfy the following relationships:

$$
\begin{aligned}
& \kappa_{1} \kappa_{2}=\left|\psi_{0101} \psi_{1001}+\psi_{1010} \psi_{0110}\right|^{2}-\left|\psi_{0011} \psi_{0000}\right|^{2} \\
& \kappa_{1}+\kappa_{2}=2 \operatorname{Re}\left(\psi_{1001}^{*} \psi_{1010}+\psi_{0101}^{*} \psi_{0110}\right) \\
& \kappa_{3} \kappa_{4}=-\left|\psi_{0101} \psi_{1001}+\psi_{1010} \psi_{0110}\right|^{2}+\left|\psi_{0011} \psi_{0000}\right|^{2} \\
&+\left|\psi_{0011} \psi_{1100}\right|^{2} \\
& \kappa_{3}+\kappa_{4}=\left|\psi_{0000}\right|^{2}+\left|\psi_{0011}\right|^{2}+\left|\psi_{1100}\right|^{2}
\end{aligned}
$$

In the second case we consider, only trilinear terms are present, and we find

$$
\begin{gathered}
\kappa_{1} \kappa_{2}=\left|\psi_{0000} \psi_{0111}\right|^{2}+\left|\psi_{0000} \psi_{1011}\right|^{2}-\left|\psi_{1110} \psi_{1101}\right|^{2}, \\
\kappa_{1}+\kappa_{2}=\left|\psi_{0000}\right|^{2}+\left|\psi_{1011}\right|^{2}+\left|\psi_{0111}\right|^{2}, \\
\kappa_{3} \kappa_{4}=\left|\psi_{1110} \psi_{1101}\right|^{2}
\end{gathered}
$$




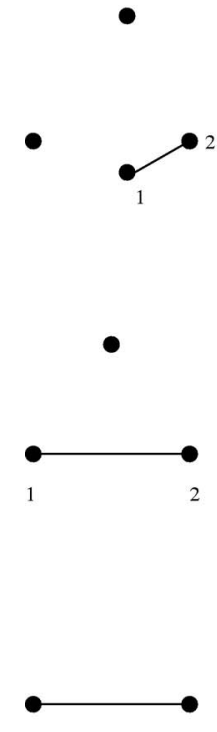

(a)
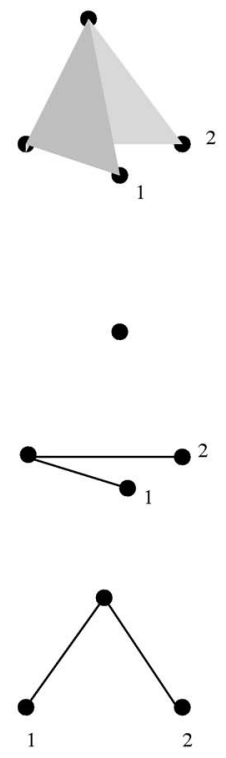

(b)
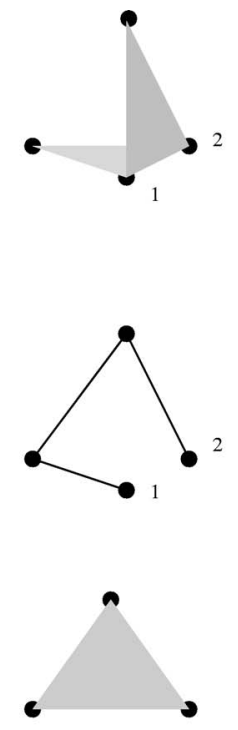

(c)
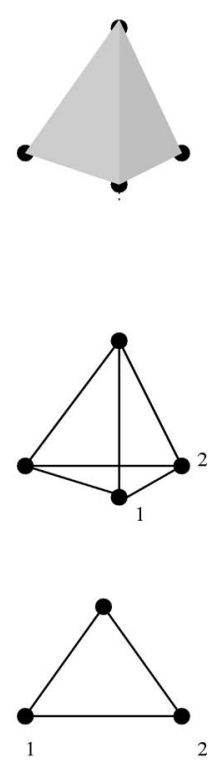

(d)
FIG. 5. Bipartite entanglement among the qubits 1 and 2. (a) Direct, (b) indirect, (c) configurations without entanglement, and (d) configurations that can result in cancellations of entanglement.

$$
\kappa_{3}+\kappa_{4}=\left|\psi_{1110}\right|^{2}+\left|\psi_{1101}\right|^{2}
$$

The considerable simplification achieved for the formulas of familiar measures can help us to construct a topological picture of entanglement based on the invariant coefficients of the canonical form. This analysis also suggests an alternative derivation of the 3-tangle.

The rules that we prescribe for constructing entanglement graphs based on the coefficients of the tanglemeter are as follows:

(i) Assign for each bilinear term $\beta_{i j}$ a line connecting the qubits $i$ and $j$.

(ii) For each trilinear term $\beta_{i j k}$, a surface on the plane confined by the $i, j, k$ qubits.

(iii) For higher order terms, volumes, among the qubits involved.

From Eqs. (B1), (29), and (B2), one sees that the existence of a bilinear term $\beta_{i j}$ in the general case implies the presence of bipartite entanglement between the qubits $i$ and $j$; we call this type of bipartite entanglement direct and represent it graphically as in Fig. 5(a). Bipartite entanglement is also present when the two qubits are indirectly connected by a line that passes through a third qubit. We can see from Eq. (29) that the concurrence $C_{12}$ is nonzero also when both $\beta_{5}, \beta_{6}$ are present, Fig. 5(b). On the other hand, there is no indirect bipartite entanglement if the line connecting the two qubits involves more than two edges, as in Fig. 5(c). In the general case, in which both direct and indirect contributions are present, there are cancellation effects instead of addition, since the closed loops contribute to tripartite entanglement (see below). There are also cases in which bipartite entanglement is due to higher-order terms, as in Fig. 5(b), where the terms $\beta_{1110}, \beta_{1101}$ are present and the eigenvalues $\kappa_{1}$ and $\kappa_{2}$ in Eq. (B3) become negative. Another configuration of surfaces, like that in Fig. 5(c), does not create bipartite entanglement.

Trilinear terms correspond to surfaces and by the Eq. (29) we see that their presence in the canonical form of three qubits results in nonvanishing 3-tangle, as in Fig. 6(a). This is not the only configuration that permits tripartite entanglement; a loop consisting of three lines is also a configuration with genuine tripartite entanglement, as in Eq. (29). The inverse statement is not true for three qubits, since the term $\beta_{7}$ is not present in the expression of concurrence.

As an application, in Fig. 6(b) we represent diagrammatically the three classes of three-qubit entanglement. It is important to note that if a diagrams is connected, i.e., there is a

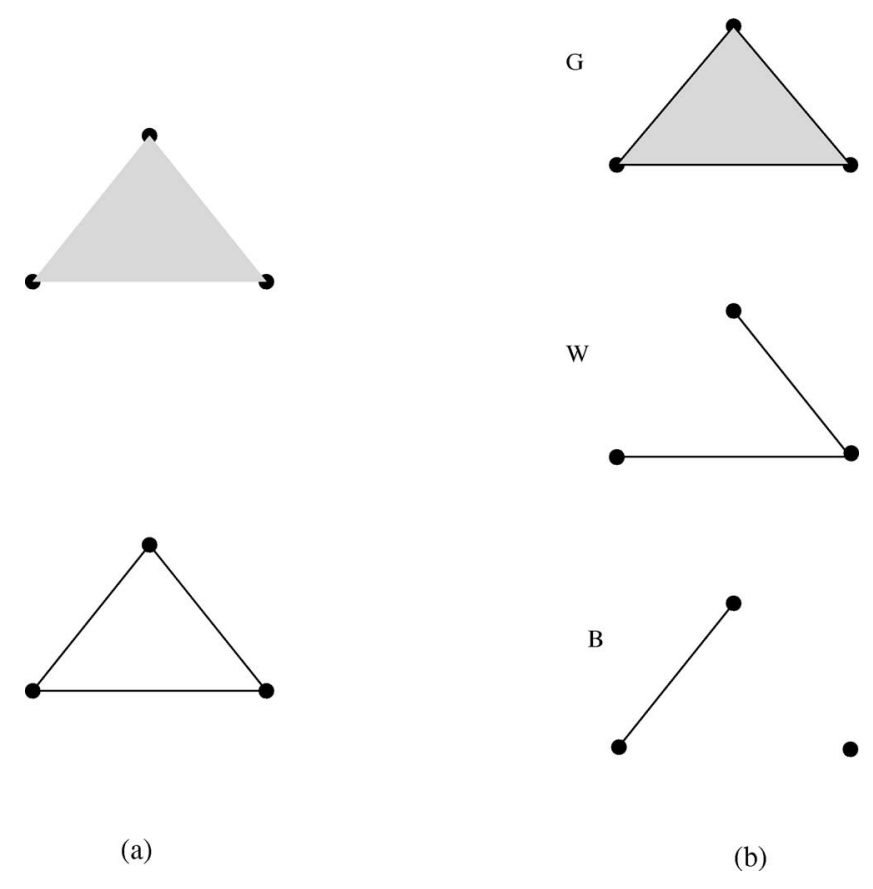

FIG. 6. (a) Tripartite entanglement in two different configurations. (b) The three classes of entanglement for three qubits. $B$ stands for biseparable, $W$ for the singular class containing the Werner state and $G$ for the the general orbit. 
closed loop or surface, then this property cannot change under general local transformations.

We anticipate that the diagrammatic technique can be generalized to more qubits and improved with directed lines, surfaces and volumes that would aid in visualizing of cancellations or joint contributions. It appears that $N$-partite entanglement can be attributed not only to the coefficients in front of the $N$ th order monomials, but also to products of lower-order coefficients.

(b) Summarizing entanglement classes under SL $(2, \mathrm{C})$ transformations: In Sec. III D 4, we have shown how the sl-entanglement classes emerge from the consideration of the tanglemeter dynamics under controlled action of continuous local transformations when proper feedback requirements are imposed. The general class has been identified along with four different singular classes, corresponding to one, two, three, and four vanishing eigenvectors of the determinant (69). All of these classes were characterized by three complex parameters. Here, we further refine this analysis and add some singular classes that depend on less parameters in order to compare this classification with that given in Ref. [24]. We illustrate the correspondence, when we normalize the states of Ref. [24] to unit vacuum amplitude and compare these states with ones suggested by sl-tanglemeters.

Let us remind that the sl-tanglemeter for four qubits we obtain in two steps. We first employ eight out of 12 complex parameters of local SL transformations and put the generic state depending initially on 15 complex parameters to the form of Eq. (27), which depends only on seven complex parameters. Then we chose the rest of the available transformations, four scaling operators $e^{B_{i} \sigma_{i}^{z}}$, in order to reduce the sl-tanglemeter to the form of Eq. (28). However, the second step not always possible to perform: when the tanglemeter is singular and one or more of the coefficients $\beta$ equal zero, the scalings can simplify the nilpotential further, although not to the form of Eq. (28). For example, when in Eq. (27) $\beta_{3}=0$, the tanglemeter can be set in the form

$$
\begin{aligned}
f_{C}= & \sigma_{3}^{+} \sigma_{4}^{+}+\beta_{5}\left(\sigma_{1}^{+} \sigma_{3}^{+}+\sigma_{2}^{+} \sigma_{4}^{+}\right)+\beta_{6}\left(\sigma_{1}^{+} \sigma_{4}^{+}+\sigma_{2}^{+} \sigma_{3}^{+}\right) \\
& +\sigma_{1}^{+} \sigma_{2}^{+} \sigma_{3}^{+} \sigma_{4}^{+},
\end{aligned}
$$

characterized by only two parameters. For $\beta_{3}=\beta_{10}=0$, it reads

$$
f_{C}=\sigma_{3}^{+} \sigma_{4}^{+}+\sigma_{1}^{+} \sigma_{3}^{+}+\beta_{6}\left(\sigma_{1}^{+} \sigma_{4}^{+}+\sigma_{2}^{+} \sigma_{3}^{+}\right)+\sigma_{1}^{+} \sigma_{2}^{+} \sigma_{3}^{+} \sigma_{4}^{+},
$$

and $\beta_{3}=\beta_{10}=\beta_{9}=0$ results in

$$
f_{C}=\sigma_{3}^{+} \sigma_{4}^{+}+\sigma_{1}^{+} \sigma_{3}^{+}+\sigma_{2}^{+} \sigma_{3}^{+}+\sigma_{1}^{+} \sigma_{2}^{+} \sigma_{3}^{+} \sigma_{4}^{+} .
$$

When the four-linear coefficient $\beta_{15}=0$ and one or more of the quadratic coefficients are also zero, the singular classes of states without four-partite entanglement emerge: the sltanglemeter of four-qubit $W$ state,

$$
f_{C}=\sigma_{3}^{+} \sigma_{4}^{+}+\sigma_{1}^{+} \sigma_{3}^{+}+\sigma_{2}^{+} \sigma_{3}^{+},
$$

belongs to one of these classes, and separable states with tanglemeters of the type

$$
f_{C}=\sigma_{3}^{+} \sigma_{4}^{+}+\sigma_{2}^{+} \sigma_{3}^{+}+\sigma_{2}^{+} \sigma_{4}^{+},
$$

and similar, belong to other.
Consider now several special cases of the singular class Eq. (75), where all four of the eigenvalues of the determinant (69) are zero. When, in addition, one or several of the trilinear coefficients equal zero, it becomes possible to rescale one or more of the bilinear coefficients to unity. Less general classes with sl-tanglemeters like

$$
\begin{gathered}
f_{C}=\sigma_{1}^{+} \sigma_{3}^{+} \sigma_{4}^{+}+\sigma_{1}^{+} \sigma_{2}^{+} \sigma_{4}^{+}+\sigma_{2}^{+} \sigma_{3}^{+} \sigma_{4}^{+}+\sigma_{1}^{+} \sigma_{2}^{+}+\beta_{5} \sigma_{1}^{+} \sigma_{3}^{+} \\
+\beta_{6} \sigma_{2}^{+} \sigma_{3}^{+}, \\
f_{C}=\sigma_{1}^{+} \sigma_{2}^{+} \sigma_{4}^{+}+\sigma_{2}^{+} \sigma_{3}^{+} \sigma_{4}^{+}+\sigma_{1}^{+} \sigma_{2}^{+}+\sigma_{1}^{+} \sigma_{3}^{+}+\beta_{6} \sigma_{2}^{+} \sigma_{3}^{+}, \\
f_{C}=\sigma_{2}^{+} \sigma_{3}^{+} \sigma_{4}^{+}+\sigma_{1}^{+} \sigma_{2}^{+}+\sigma_{1}^{+} \sigma_{3}^{+}+\sigma_{2}^{+} \sigma_{3}^{+}
\end{gathered}
$$

emerge as a result. One reveals more singular classes, when besides several zero cubic coefficients, two or more quadratic terms vanish. Two examples,

$$
\begin{gathered}
f_{C}=\sigma_{1}^{+} \sigma_{3}^{+} \sigma_{4}^{+}+\sigma_{1}^{+} \sigma_{2}^{+} \sigma_{4}^{+}+\sigma_{2}^{+} \sigma_{3}^{+} \sigma_{4}^{+}, \\
f_{C}=\sigma_{1}^{+} \sigma_{3}^{+} \sigma_{4}^{+}+\sigma_{1}^{+} \sigma_{2}^{+} \sigma_{4}^{+}+\sigma_{2}^{+} \sigma_{3}^{+},
\end{gathered}
$$

illustrate this case.

Different four-qubit classes emerging from this classification are presented in Table I. We include the general and main singular classes and omit a number of separable singular classes corresponding to product states. For the classes not symmetric under cyclic permutation of qubit indexes, this permutation is implicit. We note that our classes result from consideration of dynamic evolution that implies a series of sequential infinitesimal local operations preserving sucanonic form of the nilpotential. Therefore the situation, where some of the obtained classes turn out to be equivalent under a finite local sl-transformation, yet cannot be excluded with certainty. Keeping this in mind, it is easy to see that $G_{a}$ is identical to the $G_{a b c d}$ class that is also suggested in Ref. [35]. The class $L_{a b c_{2}}$ corresponds to $L G 2_{a}$, while $L_{a_{2} b_{2}}$ coincides with $L G 1_{a}$. After applying SL transformations on qubits 3 and 4 of the $L_{a b_{3}}$ class, the latter reduces to a form that is a singular case of the general class. The state $L_{a_{4}}$ can be set in the canonic form by flipping the second and third qubit, and then it is a special case of $G_{c}$ for $\beta_{6}=0$ and $\beta_{7}=i$. Continuing, $L_{a_{2} 0_{3 \oplus \overline{3}}}$ coincides with $S_{f}, L_{0_{7 \oplus \overline{1}}}$ with $S_{b}$, and $L_{0_{3 \oplus 1} 0_{3 \oplus 1}}$ with $S_{d}$. The singular class $L_{0_{5 \oplus 3}}$ is of the $S_{a}$ form. Thus, we can conclude that the two classifications do overlap and complement each other.

\section{APPENDIX: C EQUATION FOR LOCAL AND TWO-BODY INTERACTION}

We provide here more detail on the derivation of the dynamic equation for $f$. Consider a single gate operation applied to qubits $i$ and $j$. We cast the function $f$ in the form $f_{00}+\sigma_{i}^{+} f_{01}+\sigma_{j}^{+} f_{10}+\sigma_{i}^{+} \sigma_{j}^{+} f_{11}$, and find

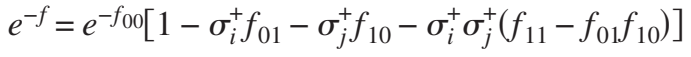

$$
\begin{aligned}
& e^{f}=e^{f_{00}}\left[1+\sigma_{i}^{+} f_{01}+\sigma_{j}^{+} f_{10}+\sigma_{i}^{+} \sigma_{j}^{+}\left(f_{11}+f_{01} f_{10}\right)\right],
\end{aligned}
$$

where the coefficients 


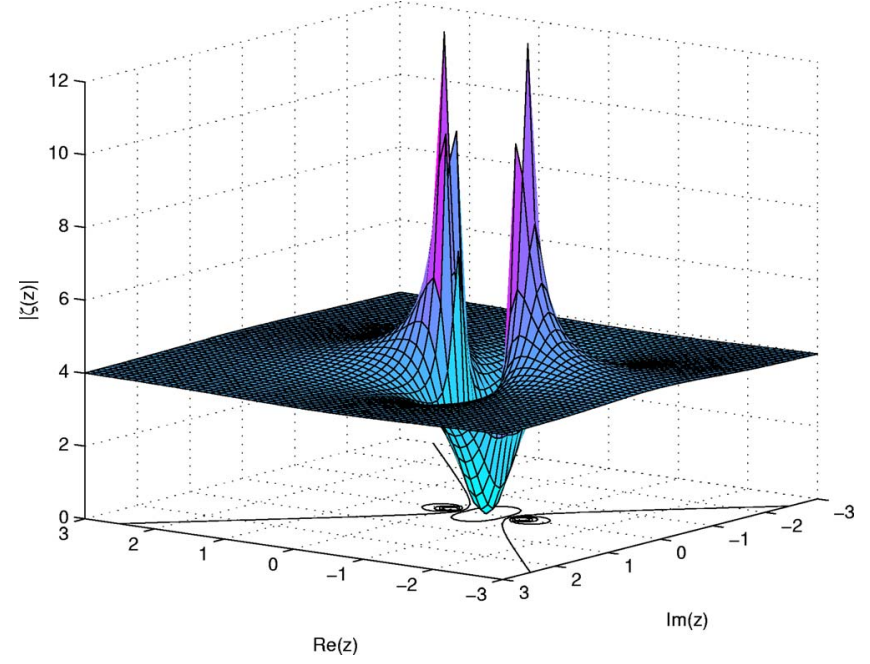

FIG. 7. (Color online) Function $|\zeta(z)|$ as in Eq. (D2). In the limit $|z| \rightarrow \infty,|\zeta(z)| \rightarrow 4$.

$$
\begin{gathered}
f_{00}=f-\sigma_{i}^{+} \frac{\partial f}{\partial \sigma_{i}^{+}}-\sigma_{j}^{+} \frac{\partial f}{\partial \sigma_{j}^{+}}+\sigma_{i}^{+} \sigma_{j}^{+} \frac{\partial^{2} f}{\partial \sigma_{i}^{+} \partial \sigma_{j}^{+}}, \\
f_{01}=\frac{\partial f}{\partial \sigma_{i}^{+}}-\sigma_{j}^{+} \frac{\partial^{2} f}{\partial \sigma_{i}^{+} \partial \sigma_{j}^{+}}, \quad f_{10}=\frac{\partial f}{\partial \sigma_{j}^{+}}-\sigma_{i}^{+} \frac{\partial^{2} f}{\partial \sigma_{i}^{+} \partial \sigma_{j}^{+}}, \\
f_{11}=\frac{\partial^{2} f}{\partial \sigma_{i}^{+} \partial \sigma_{j}^{+}},
\end{gathered}
$$

are independent of $\sigma_{i}^{+}, \sigma_{j}^{+}$. Upon substituting these equations into Eq. (49) for the Hamiltonian in Eq. (57), bearing in mind Eq. (53), we obtain

$$
\begin{aligned}
i \frac{\partial f}{\partial t}= & P_{i}^{-} \sigma_{i}^{+}+P_{j}^{-} \sigma_{j}^{+}+P_{i}^{+} \frac{\partial f}{\partial \sigma_{i}^{+}}+P_{j}^{+} \frac{\partial f}{\partial \sigma_{j}^{+}}-P_{i}^{+} \sigma_{i}^{+}\left(\frac{\partial f}{\partial \sigma_{i}^{+}}\right)^{2} \\
& -P_{j}^{+} \sigma_{j}^{+}\left(\frac{\partial f}{\partial \sigma_{j}^{+}}\right)^{2}+G_{i j}\left[\sigma_{j}^{+} \frac{\partial f}{\partial \sigma_{i}^{+}}+\sigma_{i}^{+} \frac{\partial f}{\partial \sigma_{j}^{+}}-\sigma_{i}^{+} \sigma_{j}^{+}\left(\frac{\partial f}{\partial \sigma_{i}^{+}}\right)^{2}\right. \\
& \left.-\sigma_{i}^{+} \sigma_{j}^{+}\left(\frac{\partial f}{\partial \sigma_{j}^{+}}\right)^{2}\right] .
\end{aligned}
$$

Summing over $i, j$ yields Eq. (58), where the property $G_{i j}$ $=G_{j i}$ is taken into account.

\section{APPENDIX: D DERIVATION OF 3-TANGLE BASED ON THE TANGLEMETER}

We now present a derivation of 3-tangle which is based directly on the transformations of amplitudes under local operations. The explicit expression for 3-tangle has the rather simple form of Eq. (30), when written for the su-canonical state. When considering SL transformations, we look for a quantity that takes different values within the generic sl orbit of three qubits, and vanishes outside. Performing SLtransformations, we can still set appropriate conditions so as to preserve the su-canonical form.

We start with the sl-canonical state, i.e., is the maximally entangled state $(|000\rangle+|111\rangle) / \sqrt{2}$, written in terms of nilpo- tent variables and normalizing to unit reference state amplitude,

$$
|\psi\rangle=\frac{1}{\sqrt{2}}\left(1+\sigma_{1}^{+} \sigma_{2}^{+} \sigma_{3}^{+}\right)|000\rangle .
$$

Then we apply to each qubit the general $\operatorname{SL}(2, \mathrm{C})$ transformation, which reads

$$
e^{A_{i} \sigma_{i}^{-}} e^{B_{i} \sigma_{i}^{z}} e^{C_{i} \sigma_{i}^{+}}, \quad A_{i}, B_{i}, C_{i} \in \mathbb{C}, \quad i=1,2,3 .
$$

This transformation results in the most general admissible su-canonical form of the state,

$$
\begin{aligned}
\left|\psi^{\prime}\right\rangle= & \left(\psi_{000}^{\prime}+\psi_{011}^{\prime} \sigma_{1}^{+} \sigma_{2}^{+}+\psi_{110}^{\prime} \sigma_{2}^{+} \sigma_{3}^{+}+\psi_{101}^{\prime} \sigma_{1}^{+} \sigma_{3}^{+}\right. \\
& \left.+\psi_{111}^{\prime} \sigma_{1}^{+} \sigma_{2}^{+} \sigma_{3}^{+}\right)|000\rangle,
\end{aligned}
$$

where the coefficients take the forms

$$
\begin{gathered}
\psi_{000}^{\prime}=\frac{(1+z)^{2}}{\sqrt{2}} e^{-B_{1}-B_{2}-B_{3}}, \\
\psi_{011}^{\prime}=-\frac{1+z}{z \sqrt{2}} C_{1} C_{2} e^{B_{1}+B_{2}-B_{3}}, \\
\psi_{101}^{\prime}=-\frac{1+z}{z \sqrt{2}} C_{1} C_{3} e^{B_{1}+B_{3}-B_{2}}, \\
\psi_{110}^{\prime}=-\frac{1+z}{z \sqrt{2}} C_{2} C_{3} e^{B_{2}+B_{3}-B_{1}}, \\
\psi_{111}^{\prime}=-\frac{1+2 z}{z^{2} \sqrt{2}} C_{1} C_{2} C_{3} e^{B_{1}+B_{2}+B_{3}} .
\end{gathered}
$$

Here we have employed a complex variable $z$ that naturally emerges from the requirements of su-canonical form, having the relations $z=A_{1} e^{2 B_{1}} C_{1}=A_{2} e^{2 B_{2}} C_{2}=A_{3} e^{2 B_{3}} C_{3}$ and (1 $+z)^{2} C_{2} C_{3}=-z^{2}$. One sees that the amplitudes of the sucanonical state depend on seven real parameters, since in addition to five parameters of the tanglemeter, we now allow for two more parameters characterizing the vacuum state amplitude.

Being in the general orbit, states of less general orbits can be reached if irreversible transformations are performed [35] corresponding to the limit $|z| \rightarrow \infty$. Thus, we seek to construct a polynomial measure on the amplitudes $\psi^{\prime}$, in such a way that it is a function of $z$ and it vanishes outside the general sl-orbit. Direct inspection shows that we can construct a function that depends only on the parameter $z$,

$$
\zeta(z)=\frac{\psi_{111}^{\prime 2} \psi_{000}^{\prime 2}}{\psi_{000}^{\prime} \psi_{110}^{\prime} \psi_{011}^{\prime} \psi_{101}^{\prime}}=-\frac{(1+2 z)^{2}}{z(1+z)},
$$

which is independent upon normalization. The function $\zeta(z)$ shown in Fig. 7 can take any value except -4 , which corresponds to the limit $|z| \rightarrow \infty$. Thus, the polynomial 
TABLE I. Classification of 4-qubit entanglement classes following from SL(2, C) transformation properties of the canonic form.

\begin{tabular}{|c|c|}
\hline General class & Three complex parameters \\
\hline$G_{a}$ & $\begin{array}{c}f=\beta_{3}\left(\sigma_{1}^{+} \sigma_{2}^{+}+\sigma_{3}^{+} \sigma_{4}^{+}\right)+\beta_{5}\left(\sigma_{1}^{+} \sigma_{3}^{+}+\sigma_{2}^{+} \sigma_{4}^{+}\right) \\
+\beta_{6}\left(\sigma_{1}^{+} \sigma_{4}^{+}+\sigma_{2}^{+} \sigma_{3}^{+}\right)+\sigma_{1}^{+} \sigma_{2}^{+} \sigma_{3}^{+} \sigma_{4}^{+}\end{array}$ \\
\hline Singular 3D classes & Three complex parameters \\
\hline$G_{b}$ & $\begin{array}{c}f=\beta_{3}\left(\sigma_{1}^{+} \sigma_{2}^{+}+\sigma_{3}^{+} \sigma_{4}^{+}\right)+\beta_{5}\left(\sigma_{1}^{+} \sigma_{3}^{+}+\sigma_{2}^{+} \sigma_{4}^{+}\right)+\beta_{6}\left(\sigma_{1}^{+} \sigma_{4}^{+}+\sigma_{2}^{+} \sigma_{3}^{+}\right) \\
+\sigma_{1}^{+} \sigma_{2}^{+} \sigma_{3}^{+}-\sigma_{1}^{+} \sigma_{2}^{+} \sigma_{4}^{+}+\sigma_{1}^{+} \sigma_{3}^{+} \sigma_{4}^{+}-\sigma_{2}^{+} \sigma_{3}^{+} \sigma_{4}^{+} \\
+2\left(\beta_{5} \beta_{6}-\beta_{3} \beta_{6}+\beta_{3} \beta_{5}\right) \sigma_{1}^{+} \sigma_{2}^{+} \sigma_{3}^{+} \sigma_{4}^{+}\end{array}$ \\
\hline$G_{c}$ & $\begin{array}{c}f=\sigma_{1}^{+} \sigma_{2}^{+}+\sigma_{1}^{+} \sigma_{3}^{+}+\sigma_{2}^{+} \sigma_{4}^{+}+\sigma_{3}^{+} \sigma_{4}^{+}+\beta_{6}\left(\sigma_{1}^{+} \sigma_{4}^{+}+\sigma_{2}^{+} \sigma_{3}^{+}\right) \\
+\beta_{7}\left(\sigma_{1}^{+} \sigma_{2}^{+} \sigma_{3}^{+}-\sigma_{2}^{+} \sigma_{3}^{+} \sigma_{4}^{+}\right)+\beta_{11}\left(\sigma_{1}^{+} \sigma_{2}^{+} \sigma_{4}^{+}-\sigma_{1}^{+} \sigma_{3}^{+} \sigma_{4}^{+}\right) \\
+2 \sigma_{1}^{+} \sigma_{2}^{+} \sigma_{3}^{+} \sigma_{4}^{+}\end{array}$ \\
\hline$G_{d}$ & $\begin{array}{c}f=\sigma_{1}^{+} \sigma_{2}^{+}+\sigma_{1}^{+} \sigma_{3}^{+}+\sigma_{2}^{+} \sigma_{4}^{+}+\sigma_{3}^{+} \sigma_{4}^{+}+\sigma_{1}^{+} \sigma_{4}^{+}+\sigma_{2}^{+} \sigma_{3}^{+} \\
+\beta_{14}\left(\sigma_{1}^{+} \sigma_{2}^{+} \sigma_{3}^{+}-\sigma_{1}^{+} \sigma_{2}^{+} \sigma_{4}^{+}+\sigma_{1}^{+} \sigma_{3}^{+} \sigma_{4}^{+}-\sigma_{2}^{+} \sigma_{3}^{+} \sigma_{4}^{+}\right) \\
+\beta_{13}\left(\sigma_{1}^{+} \sigma_{2}^{+} \sigma_{3}^{+}+\sigma_{1}^{+} \sigma_{2}^{+} \sigma_{4}^{+}-\sigma_{1}^{+} \sigma_{3}^{+} \sigma_{4}^{+}-\sigma_{2}^{+} \sigma_{3}^{+} \sigma_{4}^{+}\right) \\
+\beta_{11}\left(\sigma_{1}^{+} \sigma_{2}^{+} \sigma_{3}^{+}-\sigma_{1}^{+} \sigma_{2}^{+} \sigma_{4}^{+}-\sigma_{1}^{+} \sigma_{3}^{+} \sigma_{4}^{+}+\sigma_{2}^{+} \sigma_{3}^{+} \sigma_{4}^{+}\right) \\
+2 \sigma_{1}^{+} \sigma_{2}^{+} \sigma_{3}^{+} \sigma_{4}^{+}\end{array}$ \\
\hline$G_{e}$ & $\begin{array}{c}f=\sigma_{1}^{+} \sigma_{2}^{+} \sigma_{3}^{+}+\sigma_{1}^{+} \sigma_{2}^{+} \sigma_{4}^{+}+\sigma_{1}^{+} \sigma_{3}^{+} \sigma_{4}^{+}+\sigma_{2}^{+} \sigma_{3}^{+} \sigma_{4}^{+}+ \\
\beta_{3} \sigma_{1}^{+} \sigma_{2}^{+}+\beta_{6} \sigma_{2}^{+} \sigma_{3}^{+}+\beta_{5} \sigma_{2}^{+} \sigma_{3}^{+}\end{array}$ \\
\hline Singular 2D classes & Two complex parameters \\
\hline$L G 2_{a}$ & $f=\sigma_{3}^{+} \sigma_{4}^{+}+\beta_{5}\left(\sigma_{1}^{+} \sigma_{3}^{+}+\sigma_{2}^{+} \sigma_{4}^{+}\right)+\beta_{6}\left(\sigma_{1}^{+} \sigma_{4}^{+}+\sigma_{2}^{+} \sigma_{3}^{+}\right)+\sigma_{1}^{+} \sigma_{2}^{+} \sigma_{3}^{+} \sigma_{4}^{+}$ \\
\hline$L G 2_{b}$ & $f=\sigma_{1}^{+} \sigma_{2}^{+}+\sigma_{3}^{+} \sigma_{4}^{+}+\beta_{5}\left(\sigma_{1}^{+} \sigma_{3}^{+}+\sigma_{2}^{+} \sigma_{4}^{+}\right)+\beta_{6}\left(\sigma_{1}^{+} \sigma_{4}^{+}+\sigma_{2}^{+} \sigma_{3}^{+}\right)$ \\
\hline$L G 2_{c}$ & $f=\sigma_{1}^{+} \sigma_{3}^{+} \sigma_{4}^{+}+\sigma_{1}^{+} \sigma_{2}^{+} \sigma_{4}^{+}+\sigma_{2}^{+} \sigma_{3}^{+} \sigma_{4}^{+}+\sigma_{1}^{+} \sigma_{2}^{+}+\beta_{5} \sigma_{1}^{+} \sigma_{3}^{+}+\beta_{6} \sigma_{2}^{+} \sigma_{3}^{+}$ \\
\hline Singular 1D classes & One complex parameters \\
\hline$L G 1_{a}$ & $f=\sigma_{1}^{+} \sigma_{2}^{+}+\sigma_{1}^{+} \sigma_{3}^{+}+\beta_{6}\left(\sigma_{1}^{+} \sigma_{4}^{+}+\sigma_{2}^{+} \sigma_{3}^{+}\right)+\sigma_{1}^{+} \sigma_{2}^{+} \sigma_{3}^{+} \sigma_{4}$ \\
\hline$L G 1_{b}$ & $f=\sigma_{1}^{+} \sigma_{2}^{+} \sigma_{4}^{+}+\sigma_{2}^{+} \sigma_{3}^{+} \sigma_{4}^{+}+\sigma_{1}^{+} \sigma_{2}^{+}+\sigma_{1}^{+} \sigma_{3}^{+}+\beta_{6} \sigma_{2}^{+} \sigma_{3}^{+}$ \\
\hline Singular point classes & No parameters \\
\hline$S_{a}$ & $f=\sigma_{1}^{+} \sigma_{2}^{+} \sigma_{3}^{+}+\sigma_{1}^{+} \sigma_{3}^{+} \sigma_{4}^{+}+\sigma_{2}^{+} \sigma_{4}^{+}$ \\
\hline$S_{b}$ & $f=\sigma_{1}^{+} \sigma_{2}^{+} \sigma_{3}^{+}+\sigma_{1}^{+} \sigma_{3}^{+} \sigma_{4}^{+}+\sigma_{1}^{+} \sigma_{2}^{+} \sigma_{4}^{+}$ \\
\hline$S_{c}$ & $f=\sigma_{1}^{+} \sigma_{2}^{+} \sigma_{3}^{+}+\sigma_{1}^{+} \sigma_{3}^{+} \sigma_{4}^{+}$ \\
\hline$S_{d}$ & $f=\sigma_{1}^{+} \sigma_{2}^{+} \sigma_{3}^{+}$ \\
\hline$S_{e}$ & $f=\sigma_{3}^{+} \sigma_{4}^{+}+\sigma_{1}^{+} \sigma_{3}^{+}+\sigma_{2}^{+} \sigma_{3}^{+}+\sigma_{1}^{+} \sigma_{2}^{+} \sigma_{3}^{+} \sigma_{4}^{+}$ \\
\hline$S_{f}$ & $f=\sigma_{1}^{+} \sigma_{2}^{+}+\sigma_{2}^{+} \sigma_{3}^{+}+\sigma_{3}^{+} \sigma_{1}^{+}+\sigma_{1}^{+} \sigma_{2}^{+} \sigma_{3}^{+} \sigma_{4}^{+}$ \\
\hline$\cdots$ & $\cdots$ \\
\hline
\end{tabular}

$$
\left|\psi_{111}^{\prime 2} \psi_{000}^{\prime 2}+4 \psi_{000}^{\prime} \psi_{110}^{\prime} \psi_{011}^{\prime} \psi_{101}^{\prime}\right|
$$

will identically vanish outside the general orbit, and is otherwise nothing but the 3-tangle expressed in terms of the coefficients of $f_{c}$, Eq. (30).

This procedure for constructing a measure can be in principle extended to more qubits. However, the extension will require some care. The general sl-orbit of four qubits is characterized by six parameters, in contrast to the three-qubits case. Consequently, irreversible transformations may connect different sl-orbits that both contain $N$-partite entanglement. Therefore, the desired limits need to be clearly specified.

Finally, we would like to mention that Theorem 3 in Ref. [33] suggests another useful application of the $\zeta(z)$ function.
For an arbitrary 3-qubit state expressed in canonical form, one can calculate the value of $\zeta(z)$ by direct substitution of the numerical values of amplitudes to Eq. (D2), and then solve a binomial equation to find the root $z=z_{r}$. One can choose the coefficients $A_{i}, B_{i}$, and $C_{i}$ such that they satisfy $A_{i} e^{2 B_{i}} C_{i}=z_{r}$. In this way a determinant- 1 transformation that brings the maximum entangled state to the chosen state can be identified explicitly. The inverse transformation can be used for an optimal filtering procedure called entanglement distillation, a probabilistic procedure that transforms a state of the general orbit to the one with the maximal entanglement, i.e., to the GHZ state. 
[1] A. Einstein, B. Podolsky, and N. Rosen, Phys. Rev. 47, 777 (1935).

[2] E. Schrödinger, Proc. Cambridge Philos. Soc. 31, 555 (1935).

[3] M. A. Nielsen and I. L. Chuang, Quantum Computation and Information (Cambridge University Press, Cambridge, England, 2000).

[4] C. H. Bennett, G. Brassard, C. Crépeau, R. Jozsa, A. Peres, and W. K. Wootters, Phys. Rev. Lett. 70, 1895 (1993); S. L. Braunstein, G. M. D’Ariano, G. J. Milburn, and M. F. Sacchi, ibid. 84, 3486 (2000).

[5] A. K. Ekert, Phys. Rev. Lett. 67, 661 (1991); J. Kempe, Phys. Rev. A 60, 910 (1999).

[6] R. Jozsa and N. Linden, Proc. R. Soc. London, Ser. A 459, 2001 (2003).

[7] R. Orus and J. I. Latorre, Phys. Rev. A 69, 052308 (2004).

[8] A. Tal and G. Kurizki, Phys. Rev. Lett. 94, 160503 (2005); T. Opatrny, B. Deb, and G. Kurizki, ibid. 90, 250404 (2003).

[9] B. Georgeot and D. L. Shepelyansky, Phys. Rev. E 62, 3504 (2000); S. Bettelli and D. L. Shepelyansky, Phys. Rev. A 69, 054303 (2003).

[10] C. Mejia-Monasterio, G. Benenti, G. G. Carlo, and G. Casati, Phys. Rev. A 71, 062324 (2005).

[11] L. F. Santos, G. Rigolin, and C. O. Escobar, Phys. Rev. A 69, 042304 (2004).

[12] S. Montangero, G. Benenti, and R. Fazio, Phys. Rev. Lett. 91, 187901 (2003); S. Montangero and L. Viola, Phys. Rev. A 73, 040302(R) (2006).

[13] M. C. Arnesen, S. Bose, and V. Vedral, Phys. Rev. Lett. 87, 017901 (2001).

[14] T. J. Osborne and M. A. Nielsen, Phys. Rev. A 66, 032110 (2002).

[15] G. Vidal, J. I. Latorre, E. Rico, and A. Kitaev, Phys. Rev. Lett. 90, 227902 (2003).

[16] R. Eryigit, R. Eryigit, and Y. Gunduc, Int. J. Mod. Phys. C 15, 1095 (2004).

[17] P. Stelmachovic and V. Buzek, Phys. Rev. A 70, 032313 (2004).

[18] L. Amico, A. Osterloh, F. Plastina, R. Fazio, and G. M. Palma, Phys. Rev. A 69, 022304 (2004).

[19] R. Somma, G. Ortiz, H. Barnum, E. Knill, and L. Viola, Phys. Rev. A 70, 042311 (2004).

[20] T.-C. Wei, D. Das, S. Mukhopadyay, S. Vishveshwara, and P. M. Goldbart, Phys. Rev. A 71, 060305(R) (2005).

[21] T. S. Cubitt, F. Verstraete, and J. I. Cirac, Phys. Rev. A 71, 052308 (2005).

[22] D. M. Greeberger, M. Horne, A. Shimony, and A. Zeilinger, Am. J. Phys. 58, 1131 (1990).

[23] W. Dur, G. Vidal, and J. I. Cirac, Phys. Rev. A 62, 062314 (2000).

[24] F. Verstraete, J. Dehaene, B. DeMoor, and H. Verschelde, Phys. Rev. A 65, 052112 (2002).

[25] A. Peres, Phys. Rev. Lett. 77, 1413 (1996).

[26] W. K. Wootters, Phys. Rev. Lett. 80, 2245 (1998); V. Coffman, J. Kundu, and W. K. Wooters, Phys. Rev. A 61, 052306 (2000).

[27] V. Vedral, M. B. Plenio, M. A. Rippin, and P. L. Knight, Phys. Rev. Lett. 78, 2275 (1997).

[28] D. A. Meyer and N. R. Wallach, J. Math. Phys. 43, 4273 (2002).

[29] A. Wong and N. Christensen, Phys. Rev. A 63, 044301 (2001).
[30] P. Rungta, V. Buzek, C. M. Caves, M. Hillery, and G. J. Milburn, Phys. Rev. A 64, 042315 (2001).

[31] C. H. Bennett, S. Popescu, D. Rohrlich, J. A. Smolin, and A. V. Thapliyal, Phys. Rev. A 63, 012307 (2001).

[32] H. Heydari and G. Björk, J. Phys. A 37, 9251 (2004).

[33] F. Verstraete, J. Dehaene, and B. De Moor, Phys. Rev. A 68, 012103 (2003).

[34] A. Acin, A. Andrianov, L. Costa, E. Jane, J. I. Latorre, and R. Tarrach, Phys. Rev. Lett. 85, 1560 (2000); A. Acin, A. Andrianov, E. Jane, J. I. Latorre, and R. Tarrach, J. Phys. A 34, 6725 (2001).

[35] A. Miyake, Phys. Rev. A 67, 012108 (2003); A. Miyake and F. Verstraete, Phys. Rev. A 69, 012101 (2004).

[36] M. Grassl, M. Rötteler, and T. Beth, Phys. Rev. A 58, 1833 (1998).

[37] H. Barnum and N. Linden, J. Phys. A 34, 6787 (2001).

[38] M. S. Leifer, N. Linden, and A. Winter, Phys. Rev. A 69, 052304 (2004).

[39] R. M. Gingrich, Phys. Rev. A 65, 052302 (2002).

[40] A. Osterloh and J. Siewert, Phys. Rev. A 72, 012337 (2005).

[41] J. G. Luque and J. Y. Thibon, Phys. Rev. A 67, 042303 (2003).

[42] G. Vidal, J. Mod. Opt. 47, 355 (2000).

[43] C. H. Bennett, H. J. Bernstein, S. Popescu, and B. Schumacher, Phys. Rev. A 53, 2046 (1996).

[44] J.-G. Luque and J.-Y. Thibon, J. Phys. A 39, 371 (2006).

[45] H. A. Carteret, A. Higuchi, and A. Sudbery, J. Math. Phys. 41, 7932 (2000).

[46] T. A. Brun and O. Cohen, Phys. Lett. A 281, 88 (2001).

[47] A. Sudbery, J. Phys. A 34, 643 (2001); H. A. Carteret and A. Sudbery, ibid. 33, 4981 (2000).

[48] E. Briand, J.-G. Luque, and J.-Y. Thibon, J. Math. Phys. 43, 4855 (2004).

[49] The available classification relies on the structure of Dynkin diagrams. This was kindly communicated to A. M. and V. A. by Victor Kaç.

[50] N. Linden and S. Popescu, Fortschr. Phys. 46, 567 (1998).

[51] J. K. Stockton, J. M. Geremia, A. C. Doherty, and H. Mabuchi, Phys. Rev. A 67, 022112 (2003).

[52] Capital letters SU and SL will normally denote Lie groups, while the small su and sl denote the corresponding Lie algebras. However, we do not always follow this convention, unless it creates a confusion.

[53] F. A. Berezin, The Method of Second Quantization (Academic, Cambridge, 1966).

[54] K. Efetov, Supersymmetry in Disorder and Chaos (Cambridge University Press, Cambridge, 1997).

[55] A. Barenco, C. H. Bennett, R. Cleve, D. P. DiVincenzo, N. Margolus, P. Shor, T. Sleator, J. A. Smolin, and H. Weinfurter, Phys. Rev. A 52, 3457 (1995); S. Lloyd, Phys. Rev. Lett. 75, 346 (1995).

[56] J. L. Cereceda, quant-ph/0305043 (unpublished).

[57] L.-B. Fu, J.-L. Chen, and X.-G. Zhao, Phys. Rev. A 68, 022323 (2003).

[58] H. Barnum, E. Knill, G. Ortiz, R. Somma, and L. Viola, Phys. Rev. Lett. 92, 107902 (2004).

[59] H. Barnum, E. Knill, G. Ortiz, and L. Viola, Phys. Rev. A 68 , 032308 (2003).

[60] S. J. Akhtarshenas, J. Phys. A 38, 6777 (2005).

[61] Still, there is a strong indication that the maximum population is unique. Indeed, there exists a unique local unitary operation 
which, applied to a single-qubit $2 \times 2$ density matrix, maximizes its ground state population. Such transformation, applied to each qubit, maximizes the assembly ground state population.

[62] K. Vogel, V. M. Akulin, and W. P. Schleich, Phys. Rev. Lett. 71, 1816 (1993).

[63] We therefore do not address the purification protocols.

[64] D. M. Greenberger, M. Horne, and A. Zeilinger, in Bell's Theorem, Quantum Theory, and Conceptions of the Universe, edited by M. Kafatos (Kluwer, Dordrecht, 1989), pp. 69-72.

[65] Actually, a finite number of different canonic states may exist, which can be transformed into each other by SLtransformations consisting of qubit-"flips" and scaling. To be specific, we may choose one which minimizes the quantity $\left.|\ln \Sigma| \psi\right|^{2} \mid$.

[66] A mathematician would say that $\epsilon^{i i^{\prime}}$ represents an invariant tensor of both $\mathrm{SU}(2)$ and $\mathrm{SL}_{i}(2, \mathrm{C})$ transformations. On the other hand, the tensor $\delta_{i}^{j}$ is invariant under SU, but not under SL.

[67] This operation may also be defined as integration over the nilpotent variable, by the analogy to well-known anticommuting Grassman variables [53].

[68] It is worth mentioning that Eq. (62) somewhat resembles the nonlinear Schrödinger equation often encountered in quantum optics, where the presence of nonlinear terms may bring about dramatic effects, like soliton formation, creation of squeezed vacuum states, etc. In our case, however, this does not happen.

[69] M. Saffman and T. G. Walker, Phys. Rev. A 72, 022347 (2005).
[70] G. Molina-Terriza, A. Vaziri, J. Rehacek, Z. Hradil, and A. Zeilinger, Phys. Rev. Lett. 92, 167903 (2004).

[71] L. Viola, H. Barnum, E. Knill, G. Ortiz, and R. Somma, in Coding Theory and Quantum Computing, AMS Series in Contemporary Mathematics, Vol. 381, edited by D. Evans et al. (Americal Mathematical Society, Providence, RI, 2005), pp. 117-130; H. Barnum, G. Ortiz, R. Somma, and L. Viola, quant-ph/0506099.

[72] J. F. Cornwell, Group Theory in Physics (Academic, San Diego, 1997).

[73] J. E. Humphreys, Introduction to Lie Algebras and Representation Theory (Springer, New York, 1972).

[74] We emphasize that we express the state vector via commuting root vectors and not via the so-called simple root vectors, whose multiple commutators give all elements of the set $\mathcal{E}$ [for $\operatorname{su}(3)$, the simple roots are $s^{+}$and $\left.t^{+}\right]$.

[75] A. M. Perelomov, Generalized Coherent States and their Applications (Springer, Berlin, 1986).

[76] W.-M. Zhang, D. H. Feng, and R. Gilmore, Rev. Mod. Phys. 62, 867 (1990).

[77] R. Glauber, Phys. Rev. 131, 2766 (1963).

[78] Note that, however, none of these algebras are semisimple or reductive, hence care must be taken in defining notions such as Cartan decomposition, etc.

[79] See Ref. [54] for details.

[80] V. M. Akulin, Coherent Dynamics of Complex Quantum Systems (Springer, New York, 2005).

[81] M. Takahashi, Thermodynamics of One-Dimensional Solvable Models (Cambridge University Press, Cambridge, 1999). 